\title{
ULTRAFAINT DWARF GALAXIES—THE LOWEST-MASS RELICS FROM BEFORE REIONIZATION
}

\author{
Joss Bland-Hawthorn ${ }^{1}$, Ralph Sutherland ${ }^{2}$, and David Webster ${ }^{3}$ \\ ${ }^{1}$ Sydney Institute for Astronomy, School of Physics, University of Sydney, NSW 2006, Australia; jbh@physics.usyd.edu.au \\ ${ }^{2}$ Research School of Astronomy \& Astrophysics, Australian National University, Cotter Rd, Weston, ACT 2611, Australia \\ ${ }^{3}$ Sydney Institute for Astronomy, School of Physics, University of Sydney, NSW 2006, Australia \\ Received 2015 February 25; accepted 2015 May 28; published 2015 July 9
}

\begin{abstract}
New observations suggest that ultrafaint dwarf galaxies (UFDs) - the least luminous systems bound by dark matter halos $\left(\lesssim 10^{5} L_{\odot}\right)$ - may have formed before reionization. The extrapolated virial masses today are uncertain, with estimates ranging from $10^{8} M_{\odot}$ to as high as $10^{9} M_{\odot}$ depending on the assumed form of the underlying potential. We show that the progenitor halo masses of UFDs can, in principle, be as low as $M_{\mathrm{vir}} \approx 10^{7} M_{\odot}$. Under the right conditions, such a halo can survive the energy input of a supernova (SN) and its radiative progenitor. A clumpy (fractal) medium is much less susceptible to both internal and external injections of energy. It is less prone to SN sweeping (particularly if it is off-centered) because the coupling efficiency of the explosive energy is much lower than for a diffuse interstellar medium. With the aid of the 3D hydro/ionization code Fyris, we show that sufficient baryons are retained to form stars following a single SN event in dark matter halos down to $M_{\mathrm{vir}} \approx 10^{7} M_{\odot}$ in the presence of radiative cooling. In these models, the gas survives the SN explosion, is enriched with the specific abundance yields of the discrete events, and reaches surface densities where low-mass stars can form. Our highestresolution simulations reveal why cooling is so effective in retaining gas compared to any other factor. In the early stages, the super-hot metal-enriched SN ejecta exhibit strong cooling, leading to much of the explosive energy being lost. Consistent with earlier work, the baryons do not survive in smooth or adiabatic models in the event of an SN. The smallest galaxies may not contribute a large fraction of matter to the formation of galaxies, but they carry signatures of the earliest epochs of star formation, as we show. These signatures may allow us to distinguish a small primordial galaxy from one that was stripped down to its present size through tidal interaction. We discuss these results in the context of local UFDs and damped Ly $\alpha$ systems $(z \sim 2)$ at very low metallicity $([\mathrm{Fe} / \mathrm{H}] \sim-3)$. We show that both classes of objects are consistent with primordial low-mass systems that have experienced only a few enrichment events.
\end{abstract}

Key words: galaxies: dwarf - galaxies: evolution - galaxies: formation - galaxies: ISM - galaxies: photometry galaxies: stellar content

\section{INTRODUCTION}

One of the most important questions in astrophysics is what constituted the earliest baryonic systems, and whether relics of these survive to the present day. Primordial objects are important to identify at any redshift because these retain chemical signatures of the first and second generations of stars (Karlsson et al. 2013, hereafter K13). Furthermore, their existence and distribution provide important constraints on warm dark matter models (Kennedy et al. 2014) and how reionization propagated through the local universe (Busha et al. 2010; Ocvirk et al. 2013).

The earliest baryonic systems must have contained dark matter to allow gas cooling to proceed in order to form the first star (Abel et al. 2002). Gas cooling and accretion may have started as early as $z \sim 30$ in a dark matter halo with a mass of roughly $10^{5-6} M_{\odot}$. The first stars are thought to have been very massive, short-lived, and unique to their time (Yoshida et al. 2006; Gao et al. 2007). There is an emerging consensus on the sequence of events involved in forming the first stars because the hydrodynamic processes appear to be relatively well defined (Bromm \& Yoshida 2011). The same cannot be said about their chemical yields (K13). Some of these stars may have seeded the first black holes in their cores. An unknown combination of metals is expected to fall back onto the black hole seed (Podsiadlowski et al. 2002; Umeda \& Nomoto 2003), thus complicating any attempt to deduce the first stellar yields.
While many questions remain concerning the formation of the first stars (O'Shea \& Norman 2007; Norman 2010; Clark et al. 2011; Greif et al. 2011), the processes leading to "second stars" and subsequent generations are even less well understood (Ritter et al. 2012; Jeon et al. 2014). Whether the first stars inhibit or aid the formation of the second generation is a complex problem (Ahn \& Shapiro 2007). In particular, the emergence of long-lived, low-mass stars depends in part on whether dust formed from the ejected material of the first stars (Clark et al. 2008). If dust did not form in appreciable amounts, the cosmic microwave background inhibits gas cooling, in which case low-mass stars may not have emerged until $z \lesssim 10$ when mean metallicities reached $\sim 10^{-4} Z_{\odot}$ (Schneider \& Omukai 2010; Bromm \& Yoshida 2011).

The first baryonic systems. So what were the first baryonic systems? ${ }^{4}$ Were these exclusively the domain of the most massive stars, or did they include low- to intermediate-mass stars (Clark et al. 2008; Tsuribe \& Omukai 2008), and do any of these stars survive to the present day (Okrochkov \& Tumlinson 2010)? There are few if any reliable observational constraints thus far on the initial mass function (IMF) of the earliest galaxies. In this paper, our goal is to explore

\footnotetext{
4 How globular clusters fit into the early history of galaxy formation, and what they may tell us about the first stellar generations, remains a mystery. These ancient systems, which are relatively metal-enriched $([\mathrm{Fe} / \mathrm{H}] \gtrsim-2.5)$ and free of dark matter, were long considered to be the oldest baryonic relics of the early universe (Peebles \& Dicke 1968).
} 
astrophysical situations in which very low mass galaxies, i.e., baryons confined by dark matter, have survived from ancient times to the present day. These objects are of great interest because they must have preserved chemical signatures of early star formation and therefore could provide direct constraints on the properties of the earliest generations of stars (K13).

One of the most interesting developments in recent years is the discovery of ultrafaint dwarf galaxies (UFDs) in the Galactic halo (Willman et al. 2005; Belokurov et al. 2006, 2007; Zucker et al. 2006; Walsh et al. 2007). These darkmatter-dominated objects have very low baryonic mass $\left(\lesssim 10^{4}\right.$ $\left.M_{\odot}\right)$, are exceedingly faint $\left(\lesssim 10^{5} L_{\odot}\right)$, and have stellar metallicities below $[\mathrm{Fe} / \mathrm{H}]=-2$ (Martin et al. 2008). Remarkably, some UFDs are less luminous than globular clusters, but the former have dark matter whereas the latter do not, and hence must be considered as galaxies. The characteristic halo mass of UFDs today is highly uncertain, with estimates ranging from $10^{8}$ to $10^{9} M_{\odot}$ (Simon \& Geha 2007; Martin et al. 2008; Strigari et al. 2008; Bovill \& Ricotti 2009; Wolf et al. 2010; Frebel et al. 2014).

The reionization epoch. UV radiation from a dispersed population of "first stars" can dissociate $\mathrm{H}_{2}$, the primary coolant responsible for cooling within dark halos. The diffuse ionized gas is then too warm to stay bound to low-mass halos. In order for gas to be confined, the dark-halo mass must have exceeded $M_{\text {crit }} \approx 10^{8.5} M_{\odot}$ if the gas is to cool and eventually form stars after reionization has taken hold (Ikeuchi 1986; Rees 1986; Efstathiou 1992; Barkana \& Loeb 1999). These simple models based on ionization balance and heating are supported by 3D numerical simulations (Okamoto et al. 2008). Thus, knowing the characteristic halo mass of UFDs - at the time of formation-is important for ascertaining whether they formed before or after the onset of reionization.

A more nuanced approach to reionization considers not only the impact of positive feedback but also the local environment of the dwarf population. In particular, reionization must have been very patchy in the local universe owing to the clumpy distribution of the Local Group, nearby groups, and the Virgo cluster. Just how the expanding Strömgren spheres from the early galaxies evolved and overlapped is difficult to establish and presumably awaits future observations of the distribution of neutral gas at high redshift. Numerical simulations reveal that local reionization may have started around $z=11-14$ and have completed by $z=6-8$ (Busha et al. 2010; Ocvirk et al. 2013), a delay of the order of $400 \mathrm{Myr}$. A major uncertainty is the impact of the ionization front from Virgo: if the first stars formed significantly earlier, the local reionization epoch may have been earlier still. Regardless, much of the dwarf population (and presumably the globular cluster population) may have acquired most of their stars before reionization was complete (Ricotti \& Gnedin 2005; Busha et al. 2010). Indeed, their star formation may have been curtailed by the rising local UV background at late times.

The origin of UFDs. The nature of UFDs, whether they precede or follow the epoch of reionization, is an area of active research (Brown et al. 2014; Weisz et al. 2014). It is difficult to relate low-mass galaxies today with their early universe counterparts. Some UFDs may be the dynamically stripped cores of more massive systems, although the majority, as presented here, may be relics that have preserved their halo mass over billions of years, with significant accretion of dark matter at later times. (It is conceivable that the inner halo UFDs have lost the matter they accreted after $z=10$ through tidal stripping, such that the halo masses inferred today broadly reflect their masses at the time of formation.)

The mass of the proto-Galaxy at $z=10$ would have been of roughly $5 \times 10^{9} M_{\odot}$ with a virial radius of about $5 \mathrm{kpc}$. This early system was surrounded by hundreds of subhalos with masses $\lesssim 10^{7} M_{\odot}$. Over cosmic time, a small fraction of halos on highly radial orbits would have been disrupted and absorbed by the evolving Galaxy. Their orbit distribution and mass ratio compared to the progenitor halo imply that dynamical friction is relatively unimportant; thus, many are likely to survive to the present day. Some fraction of these would have accreted gas (Susa \& Umemura 2004a, 2004b), although the details will depend on many environmental factors (Benítez-Llambay et al. 2013). It is likely that the present population of a few dozen UFDs is a small fraction of a much bigger population to be revealed in future deep photometric surveys (Bullock et al. 2010; Hargis et al. 2014).

In an important new study of six UFDs (Brown et al. 2014), five objects are found to have formed $75 \%$ of their stellar mass before $z \sim 10(13.2 \mathrm{Gyr}$ ago) and all of their stars by $z \sim 3$ (11.6 Gyr ago). There is a strong suggestion here of rapid quenching by a photoionizing event: it is plausible that these UFDs preceded the main epoch of local or possibly global reionization (see also Webster et al. 2015a). In the work that follows, this is our starting assumption. We perform hydrodynamical simulations of early progenitors of UFDs with the aim of deriving their dynamical, photometric, and chemical properties today. We also consider whether our models are able to explain the new class of very metal-poor, damped Ly $\alpha$ systems (DLAs) at $z \sim 2$, which have been argued to be intrinsically low-mass systems (Cooke et al. 2011b, 2015), although rather less is known about these intriguing systems. Where our work differs from earlier research is that we focus on the retention of baryons-rather than disruption-in dark matter halos that are traditionally considered to be too small to retain baryons. This requires most of the action to take place before reionization, and in clumpy media that are less susceptible to supernova (SN) feedback.

New models. In our new low-mass halo models presented below, we consider the recent claim that star formation in UFDs finished before reionization (Brown et al. 2014), although the isochrone ages are sufficiently uncertain that some of it may have continued long after (Weisz et al. 2014). For star formation to have started so soon, some gas must have cooled and settled into the potential well before the onset of reionization. We stress that complete reionization does not completely exclude ongoing star formation for masses below $M_{\text {crit }}$ (e.g., Dijkstra et al. 2004; Susa \& Umemura 2004a, 2004b). For it to continue to later times, the nuclear gas density must be high enough to shield the central regions from a global ionizing event.

Consider the time it takes for the gas to evaporate from the low-mass halos. For a cosmic ionizing UV intensity $J_{0}=10^{-21}$ erg $\mathrm{cm}^{-2} \mathrm{~s}^{-1} \mathrm{~Hz}^{-1} \mathrm{sr}^{-1}$, with a moderate power-law form of $f_{\nu} \propto \nu^{-2}$, we obtain an ionization flux of $\sim 2 \times 10^{5}$ photons $\mathrm{s}^{-1} \mathrm{~cm}^{-2}$. The rate of evaporation of hydrogen atoms off the surface of a confined gas cloud, of radius $50 \mathrm{pc}\left(\sim 3 \times 10^{41}\right.$ $\mathrm{cm}^{2}$ ), assuming that one ionizing photon evaporates one hydrogen atom, is then given by

$$
\dot{m}_{\text {evap }} \sim 1.5 \times 10^{-3}\left(n_{\mathrm{r}}+1\right)^{-1} M_{\odot} \mathrm{yr}^{-1},
$$


where the $n_{\mathrm{r}}$ term allows for the number of recombinations in the escaping wind. Thus, the timescale for evaporation for the $M_{\mathrm{b}} \sim 10^{5} M_{\odot}$ of gas in the models shown here $\left(\tau_{\text {evap }} \sim f_{\mathrm{b}} M_{\mathrm{b}} / \dot{m}_{\text {evap }}\right)$ can exceed $100 \mathrm{Myr}$, which is long enough for star formation to proceed in the interior while the outer regions are evaporating. With reference to positive feedback processes, various authors have noted that $\mathrm{H}_{2}$ formation is enhanced in the limit of a weak ionizing intensity reaching the dense gas core (Kang \& Shapiro 1992; Tajiri \& Umemura 1998; Susa \& Umemura 2000, 2004a), which can further accelerate star formation. Some of these processes may assist ongoing star formation in UFDs after the onset of reionization (Brown et al. 2014; Weisz et al. 2014).

For dwarf galaxies in the outer halo at the time of their formation, their internal radiation fields are likely to have dominated local reionization (Ocvirk \& Aubert 2011). Here we consider the impact of UV radiation from massive young stars born within the low-mass halo and the impact of the subsequent $\mathrm{SN}$ explosion on gas retention. An alternative route to suppressing star formation in low-mass systems is to remove or heat up the gas through starburst-driven winds (Mac Low \& Ferrara 1999). In their 2D axisymmetric models, constant energy input corresponding to central explosions is found to couple efficiently to a smooth gas component confined by a flattened disk. Once again, these models rule out any gas surviving in a dark matter halo with a mass $\lesssim M_{\text {crit }}$. But these authors did not consider discrete SN events and modeled the winds moving through a smooth medium rather than a more physical clumpy medium where the hot wind fluid couples less efficiently with the dense interstellar medium (ISM; Cooper et al. 2008). We consider the impact of winds in smooth and clumpy media and winds that are off-centered within the galaxy. Both off-centered explosions and clumpy media are likely at any epoch, and therefore both cases must be considered in detail.

Here we investigate the conditions under which a "minimum-mass" system confined by a dark matter halo, specifically a relic of the early universe, survives to the present day. We consider feedback arising from high-mass stars due to their stellar winds, their radiation fields, and their subsequent SN explosions. We find that these limiting cosmological systems may have had total masses of $M_{\min } \sim 10^{7} M_{\odot}$, well below the limit of what is thought to have survived the reionization epoch. In our follow-up papers, we perform new calculations of chemical evolution within these "minimum-mass" galaxies over cosmic time (Webster et al. 2014, 2015a, 2015b). Conceivably, one can hope to learn about the star formation before, during, and immediately after the reionization epoch from distinct chemical signatures in these low-mass systems (e.g., Bland-Hawthorn et al. 2010).

In our new models, the initial baryon fraction is roughly $f_{\mathrm{b}} \approx 10 \%$ of the virial mass, although the final baryon fraction can be orders of magnitude smaller. This is consistent with observations of dark-matter-dominated systems observed today in the low-mass limit (McGaugh et al. 2010). As we shall see, our new models explain some UFDs (Willman et al. 2005; Irwin et al. 2007; Simon \& Geha 2007; Martin et al. 2008; Strigari et al. 2008) and the peculiar chemical signatures observed in very metal-poor DLAs (Pettini et al. 2008; Penprase et al. 2010; Cooke et al. 2011a). We discuss our new simulations in light of both classes of objects (see also Salvadori \& Ferrara 2009, 2012).
In Section 2, we describe the scope of our numerical experiments. The model parameters are summarized in Section 3. In Section 4, we introduce the simulations, and we present our results in Section 5. In Section 6, we discuss these results in light of new observations, before giving the conclusions in Section 7.

\section{STATEMENT OF THE PROBLEM}

\subsection{What Is a First Galaxy?}

Our interest in galaxy systems with the lowest possible mass arises from a desire to understand the chemistry of the first stars (Frebel \& Bromm 2012; Frebel et al. 2014, K13). Our assumption is that the surviving remnants of the first galaxies will have small dark halo masses and possibly low baryon mass fractions for the reasons outlined in Section 1. Many of these systems will have merged with larger galaxies long ago, but some are likely to be falling into larger systems today for the first time.

So what constitutes a first galaxy? This must retain a longlived stellar system and importantly a confining dark matter halo. ${ }^{5}$ Whether low-mass stars formed alongside or immediately after the first stars is unknown. It is likely that the process was patchy, with the very first stars forming in rare density peaks, but with an increasing fraction of the universal volume becoming ionized as the first stellar generation took hold.

Here we make the simple assumption that the universe was enriched to a threshold metallicity of order $[\mathrm{Fe} / \mathrm{H}]=-4$ before reionization was complete (e.g., Bromm \& Yoshida 2011). Some of this gas was accreted onto low-mass dark matter halos, and star formation began. Our threshold metallicity allows us to consider a "normal" IMF in order to describe how star formation proceeded at these early redshifts (Bromm et al. 2001; Schneider et al. 2002). Given the stochasticity of star formation at our low masses, there may be a significant amount of low-mass star formation before the first SN event. Some remnants of first galaxies may therefore contain stars formed from gas enriched only by Population III stars, thereby providing direct information on the first stellar yields.

However, in the systems relevant to our work (UFDs, very metal-poor DLAs), some of the detected elements (e.g., Sr) can only have arisen from SN ejecta. Thus, our minimum-mass models have the more stringent requirement that they can survive one or more $\mathrm{SN}$ events during their lifetime. In order to experience more than a single star formation episode, a very small galaxy must retain enough gas in the face of the energy outflow from a single star-forming episode for a second one to occur, perhaps at a much later time. A large burst of energy such as an SN could either heat the galaxy ISM and halt star formation or completely evacuate the star formation region of gas. This will cut off star formation until such time as the halo re-accretes the blown-out gas, assuming that this is allowed by the environment. Thus, a "first galaxy" is one that must retain sufficient gas to form stars in the wake of at least a single SN event.

This is not a contrived circumstance, and we show below (Section 6) that there may already be observations to support such a scenario. It is likely that there are situations in which only one or two SNe occur within a single star-forming "burst" (Karlsson et al. 2013; Ritter et al. 2014). Hurley-Keller et al. (1998) identify several starburst epochs in the Carina dwarf,

\footnotetext{
5 We recognize that "tidal dwarf galaxies" form a rare class of objects that may not have associated dark matter, but generally a galaxy is defined by the existence of a confining dark matter halo, at least at the time of formation.
} 


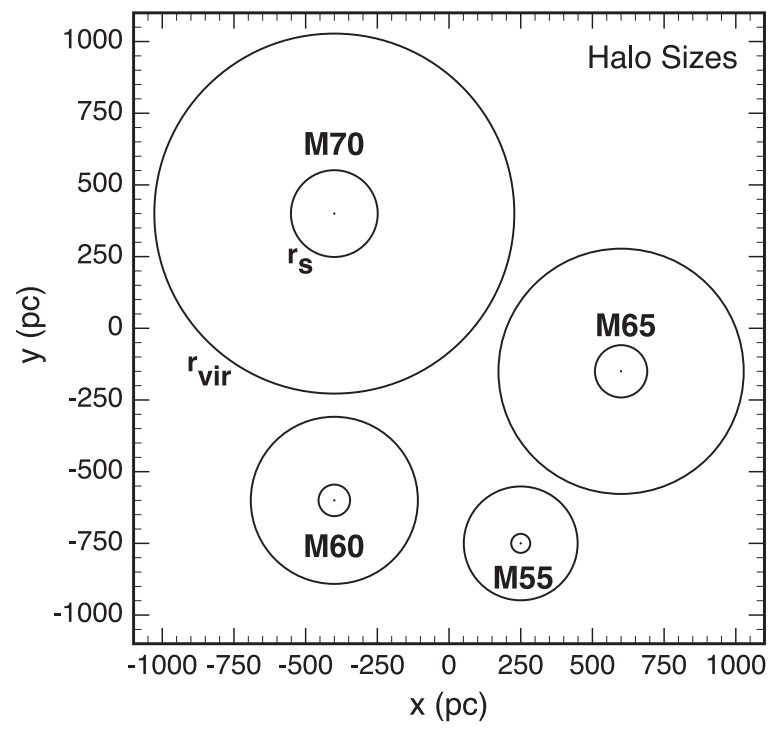

Figure 1. Einasto dark matter halos considered here showing the relative sizes of the virial and scale radii at $z=10$. The virial masses of the four halos are $\log \left[M_{\mathrm{vir}}\left(M_{\odot}\right)\right]=5.5,6.0,6.5$, and 7.0; we refer to these models as M55, M60, M65, and M70, respectively. For all halos, the virial mass and radius continue to grow to $z=0$ (see Figure 2).

with the first of these taking place of order $\sim 10 \mathrm{Gyr}$ ago. At that time, the surface density of the star formation rate $\left(s_{\star}\right)$ within the core was roughly $10^{-3} M_{\odot} \mathrm{yr}^{-1} \mathrm{kpc}^{-2}$ over the period of the burst, $T_{0}$. This timescale is now well established in starburst and dwarf galaxies such that $T_{o} \lesssim 10 \mathrm{Myr}$ (Tolstoy et al. 2009; Sharp \& Bland-Hawthorn 2010).

\subsection{Star Formation and $\mathrm{SNe}$}

To test the plausibility of a single SN event occurring in our models, we consider a simple model of star formation. We consider dark matter potentials with an Einasto profile over the virial mass range $\log \left[M_{\text {vir }}\left(M_{\odot}\right)\right]=5.5,6.0,6.5$, and 7.0; we refer to these models as M55, M60, M65, and M70, respectively (see Figure 1). If we integrate over the Einasto profile, the total masses for these models in dark matter are $\log \left[M_{\text {tot }}\left(M_{\odot}\right)\right]=5.9,6.4,7.0$, and 7.5. (These total masses do not include the small amount of baryons.) In Figure 2, we show how the low-mass halos are expected to grow in mass and radius with cosmic time. Halo growth is not treated in our simulations because the time frame of our analysis is short. Our results at a given halo mass at $z=10$ correspond to more massive systems at later times.

Here we show complete results for M65 and M70 and partial results for M55 and M60 because these models fail to retain sufficient baryons. The initial baryon fraction is assumed to be roughly $10 \%$ within the virial radius (e.g., Krumholz \& Dekel 2012). We assume that star formation occurs where the gas is densest, i.e., within a radius enclosing $50 \%$ of the baryons $\left(r_{0.5 g}\right)$ inside one scale radius $r_{\mathrm{s}}$, the scale radius of the Einasto potential (see Section 3.1). Over this volume, we find that the gas density is within a factor of 6 of the maximum central value.

For the models (M55, M60, M65, M70), the enclosed gas masses within the half gas-mass radius $\left(\Sigma_{s} / 2\right)$ are $\left(2.8 \times 10^{3}\right.$, $\left.9.5 \times 10^{3}, 2.5 \times 10^{4}, 7.5 \times 10^{4}\right) M_{\odot}$, and thus the average gas surface densities are $(2.1,2.6,3.3,4.2) M_{\odot} \mathrm{pc}^{-2}$. In the clumpy gas models, there are order-of-magnitude variations in local gas density. From direct observation, we know that star formation

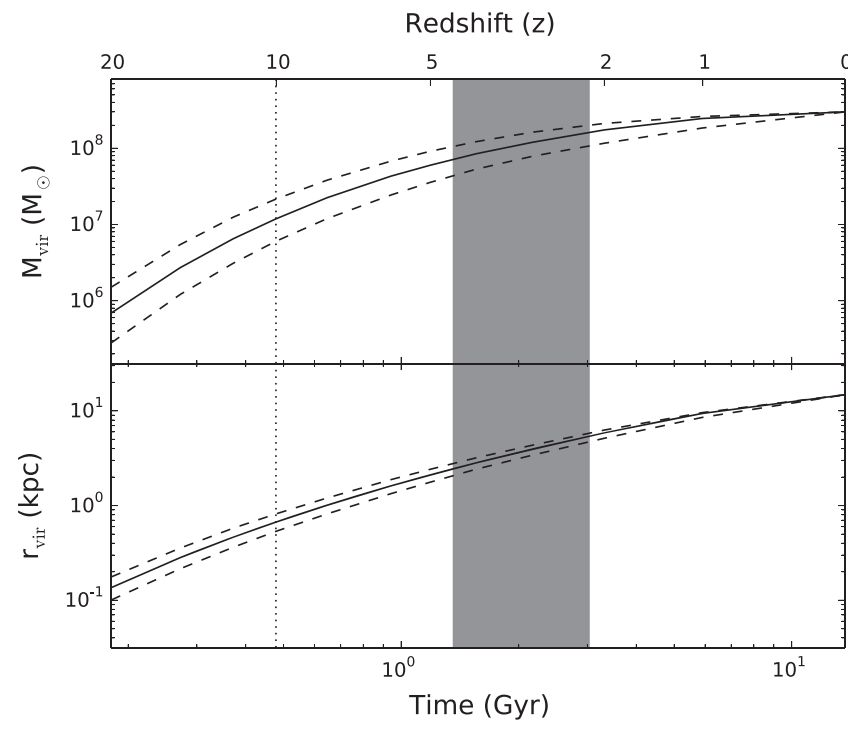

Figure 2. Predicted growth in the virial mass (top) and radius (bottom) of the M70 halo at $z=10$ (dotted line) to the present day (see Figure 1). The shaded band is the redshift range of the DLAs discussed in later sections. This plot was derived from 5000 runs of the tree merger code of Parkinson et al. (2008): the dashed lines encompass $67 \%$ of the predicted halos at each epoch.

takes place at these moderately low column densities over a range of metallicities down to $[\mathrm{Fe} / \mathrm{H}] \approx-1$ (e.g., Bigiel et al. 2008). As these authors show, the star formation efficiency is observed to vary wildly below $9 M_{\odot} \mathrm{pc}^{-2}$, with a median value of only a few percent. Little is known about how the star formation rate depends on metallicity, but it seems likely that it declines with $[\mathrm{Fe} / \mathrm{H}]$ owing to the difficulty of forming $\mathrm{H}_{2}$. We stress, however, that the inferred value of $s_{\star}$ in Carina is comparable to what is observed in the outer metalpoor regions of spiral galaxies (e.g., Bigiel et al. 2008), and this dwarf galaxy somehow managed to form long-lived stars at much lower metallicities.

For a star formation efficiency of $2 \%$ (Kennicutt 1989), the expected total stellar mass over the lifetime of the system is $(56,190,670,2400) M_{\odot}$. If we assume a Kroupa mass function, roughly one-fifth of the mass in stars will end their lives as SNe. These estimates are susceptible to a lot of scatter in the low-mass/surface density limit (Bigiel et al. 2008). If we scale the projected star formation rate per unit area to the largest burst in Carina $\left(\sim 10^{-3} M_{\odot} \mathrm{yr}^{-1} \mathrm{kpc}^{-2}\right)$, the timescales required to form these stellar masses are $(44,54,71,86) \mathrm{Myr}$, for a half gas-mass radius of $(20,34,55,94)$ pc (see Section 3.1 for further details on the halo properties). If a typical burst lasts $10 \mathrm{Myr}$, as in Carina (Tolstoy et al. 2009), we would expect multiple bursts over the star-forming lifetime of the galaxy.

So can we consider an isolated SN event to be applicable to all of our models? It is conceivable that more than one event can occur within the timescale of a single burst $T_{o}$. The results given in Table 1 show that multiple SN events are rare in the M55 and M60 models and are relatively unusual in the M65 model, although they occur half the time in the M70 model. Multiple SNe in more gas-rich M70 models happen half the time, but in practice we can consider most of these eventualities to be "single SN events" because one of these is highly likely to be a low-mass event and physically separated from the other event. These events are likely to enrich their own immediate environs, although substantial mixing can occur between the 
Table 1

Likelihood of Supernova Events Scaled to Carina Star Formation Rate for a Typical Burst Duration Lasting $10 \mathrm{Myr}$

\begin{tabular}{lcccc}
\hline \hline & M55 & M60 & M65 & M70 \\
\hline 0 & $93 \%$ & $80 \%$ & $45 \%$ & $19 \%$ \\
1 & $6.6 \%$ & $18 \%$ & $36 \%$ & $32 \%$ \\
$>1$ & $0.2 \%$ & $2.0 \%$ & $19 \%$ & $50 \%$ \\
\hline \multirow{2}{*}{$t_{\text {SN }}(\mathrm{Myr})$} & 100 & 30 & 8 & 4 \\
\hline
\end{tabular}

Note. $t_{\mathrm{SN}}$ is the median length of time between SNe in Myr.

events at late times (Ritter et al. 2014). While different choices of the size of the star-forming region or baryon fraction may alter these results, we would not expect this to alter the overall conclusion of a single SN event. We note that simulations of more massive halos will need to consider multiple events within the same burst.

\subsection{The Pre-SN Phase}

Massive stars evolve rapidly in the lead-up to the explosion, during which time the stellar wind and radiative luminosity can be substantial. This pre-SN phase can be an important factor in removing gas, particularly for halos with low baryon fractions. In the worst-case scenario, a star at the halo center can ionize an amount of gas, $M_{\text {ion }} \sim S_{\star} m_{\mathrm{p}} / \alpha n_{\mathrm{H}} M_{\odot}$, where $\alpha$ is the total recombination coefficient, $m_{\mathrm{p}}$ is the mass of the proton, and $n_{\mathrm{H}}$ is the hydrogen density. Using the Schaerer (2003) models at a metallicity of $[\mathrm{Fe} / \mathrm{H}]=-4.0$, the mean ionizing rate averaged over the Kroupa IMF is $S_{\star} \sim 4 \times 10^{48}$ photons s ${ }^{-1}$ for the M55 model, $1 \times 10^{49}$ photons $\mathrm{s}^{-1}$ for M60, and $3 \times 10^{49}$ photons s ${ }^{-1}$ for M65. Taking the worst-case scenario where the most massive star falls near the center, the total ionized masses are $1.7 \times 10^{3} M_{\odot}$ for M55, $6.1 \times 10^{3} M_{\odot}$ for M60, $2.2 \times 10^{4}$ $M_{\odot}$ for M65, and $5.5 \times 10^{4} M_{\odot}$ for M70. In each case, a large fraction of the neutral gas can be ionized. This is likely to have a significant impact in reducing subsequent star formation. But the more likely situation is not expected to be so severe. Because the total amount of star formation is small in our models, stochastic effects can dominate. The median radiation levels expected are more biased to B stars rather than rare $\mathrm{O}$ stars, and the ionized masses can be much less than $10 \%$. We include the effects of preionization in our models below.

\section{MODEL PARAMETERS}

\subsection{Numerical Models}

In our experiments, we look at a series of spherically symmetric gas distributions in hydrostatic equilibrium, detonate a single $\mathrm{SN}$, and track the ability of the potential to retain significant amounts of gas over $25 \mathrm{Myr}$ or more. The parameters of our models are shown in Table 2. For all of our models, we consider the period prior to the onset of cosmological reionization when stars were forming for the first time. At such early times, substantial amounts of gas have already begun to settle into dark matter halos up to and exceeding $M_{\text {crit }}$ (Bullock et al. 2001; Ricotti \& Gnedin 2005). This is consistent with the latest results from deep photometry for UFDs, where star formation appears to have been quenched by reionization in almost all cases (Brown et al. 2012, 2014; Webster et al. 2015a).
Table 2

Model Parameters

\begin{tabular}{lcccc}
\hline \hline \multicolumn{5}{c}{ Cosmology } \\
\hline & $z$ & $h$ & $\Omega_{\Lambda}$ & $\Omega_{M}$ \\
& 10.0 & 0.7 & 0.7 & 0.3 \\
$\alpha$ & $\Delta_{c}$ & $\rho_{c}\left(\mathrm{~g} \mathrm{~cm}^{-3}\right)$ & $\Delta_{u}$ & $\left\langle\rho_{u}\right\rangle\left(\mathrm{g} \mathrm{cm}^{-3}\right)$ \\
0.18 & 177.5 & $3.68 \mathrm{E}-027$ & 591.7 & $1.11 \mathrm{E}-027$ \\
\hline
\end{tabular}

Dark Matter

\begin{tabular}{|c|c|c|c|c|}
\hline Model & $\begin{array}{c}r_{\mathrm{s}} \\
(\mathrm{pc})\end{array}$ & $\begin{array}{c}\rho_{\mathrm{s}} \\
\left(\mathrm{g} \mathrm{cm}^{-3}\right)\end{array}$ & $x_{\mathrm{vir}}$ & $\begin{array}{l}r_{\mathrm{vir}} \\
(\mathrm{pc})\end{array}$ \\
\hline M55 & 33.2 & $1.02 \mathrm{E}-023$ & 5.98 & 198.5 \\
\hline M60 & 55.0 & $7.73 \mathrm{E}-024$ & 5.29 & 291.3 \\
\hline M65 & 91.3 & $5.87 \mathrm{E}-024$ & 4.69 & 427.6 \\
\hline M70 & 151.3 & 4.48E-024 & 4.15 & 627.7 \\
\hline Model & $\begin{array}{c}M_{\mathrm{s}} \\
\left(M_{\odot}\right)\end{array}$ & $\begin{array}{c}M_{\mathrm{vir}} \\
\left(M_{\odot}\right)\end{array}$ & $\begin{array}{l}M_{300} \\
\left(M_{\odot}\right)\end{array}$ & $\begin{array}{c}M_{\mathrm{tot}} \\
\left(M_{\odot}\right)\end{array}$ \\
\hline M55 & $5.64 \mathrm{E}+04$ & $3.16 \mathrm{E}+05$ & $4.10 \mathrm{E}+05$ & $7.90 \mathrm{E}+05$ \\
\hline M60 & $1.94 \mathrm{E}+05$ & $1.00 \mathrm{E}+06$ & $1.02 \mathrm{E}+06$ & $2.73 E+06$ \\
\hline M65 & $6.73 E+05$ & $3.16 \mathrm{E}+06$ & $2.36 \mathrm{E}+06$ & $9.44 \mathrm{E}+06$ \\
\hline M70 & $2.34 \mathrm{E}+06$ & $1.00 \mathrm{E}+07$ & $5.08 \mathrm{E}+06$ & $3.29 \mathrm{E}+07$ \\
\hline \multicolumn{5}{|c|}{ Baryons } \\
\hline Model & $\begin{array}{c}c_{\mathrm{s}} \\
\left(\mathrm{km} \mathrm{s}^{-1}\right)\end{array}$ & $\begin{array}{c}c_{\mathrm{s}}, \text { iso } \\
\left(\mathrm{km} \mathrm{s}^{-1}\right)\end{array}$ & $\begin{array}{l}T_{\text {gas }} \\
(\mathrm{K})\end{array}$ & $\begin{array}{c}M_{\text {gas, vir }} \\
\left(M_{\odot}\right)\end{array}$ \\
\hline M55 & 2.70 & 1.91 & $5.33 \mathrm{E}+02$ & $3.87 \mathrm{E}+04$ \\
\hline M60 & 3.90 & 2.76 & $1.11 \mathrm{E}+03$ & $1.19 \mathrm{E}+05$ \\
\hline M65 & 5.63 & 3.98 & $2.32 \mathrm{E}+03$ & $3.66 \mathrm{E}+05$ \\
\hline \multirow[t]{2}{*}{ M70 } & 8.16 & 5.77 & $4.86 \mathrm{E}+03$ & $1.15 \mathrm{E}+06$ \\
\hline & $\begin{array}{l}r_{0.5 \mathrm{~g}} \\
(\mathrm{pc})\end{array}$ & $\begin{array}{c}\rho_{\mathrm{gas}, 0} \\
\left(\mathrm{~g} \mathrm{~cm}^{-3}\right)\end{array}$ & $\begin{array}{c}n_{\mathrm{H}, 0} \\
\left(\mathrm{~cm}^{-3}\right)\end{array}$ & $\begin{array}{l}M_{\mathrm{gas}, \mathrm{s}} \\
\left(M_{\odot}\right)\end{array}$ \\
\hline M55 & 20.8 & $4.09 \mathrm{E}-23$ & 17.2 & $5.63 \mathrm{E}+03$ \\
\hline M60 & 34.5 & $3.11 \mathrm{E}-23$ & 13.1 & $1.94 \mathrm{E}+04$ \\
\hline M65 & 57.1 & $2.36 \mathrm{E}-23$ & 9.91 & $6.73 \mathrm{E}+04$ \\
\hline M70 & 94.8 & $1.79 \mathrm{E}-23$ & 7.55 & $2.34 \mathrm{E}+05$ \\
\hline
\end{tabular}

Note. The cosmology is fixed by the halo formation redshift, $z$, the normalized hubble constant $h=H_{o} / 100 \mathrm{~km} \mathrm{~s}^{-1} \mathrm{Mpc}^{-1}$, the dark energy density $\Omega_{\Lambda}$, and the dark matter density $\Omega_{M}$.

The Einasto halo properties (see text) are fixed by the concentration parameter, $\alpha$, and the halo dark matter density contrast with the ambient medium, $\Delta$, defined at redshift $z$. This density contrast can be expressed as $\Delta_{c}$ averaged over the virial radius and normalized to the mean critical density $\rho_{c}$, or as $\Delta_{u}$ normalized to the universal mass density $\left\langle p_{u}\right\rangle$. The halo potential is defined by its scale radius, $r_{\mathrm{s}}$, and the mean density and mass within this radius, $\rho_{\mathrm{s}}$ and $M_{\mathrm{s}}$. The halo virial mass is $M_{\mathrm{vir}}$ within the virial radius $r_{\mathrm{vir}}=x_{\mathrm{vir}} r_{\mathrm{s}} ; M_{300}$ is the mass within $300 \mathrm{pc}$, and $M_{\text {tot }}$ is the mass integrated to infinity. For the Baryons, $c_{\mathrm{s}}$ and $c_{\mathrm{s}, \text { iso }}$ are the adiabatic (physical) and isothermal sound speeds in the gas with mean temperature $T_{\text {gas }}$. This gas has a central density (number density) of $\rho_{\text {gas }, 0}\left(n_{H, 0}\right)$ with a virial (total) mass of $M_{\text {gas,vir }}\left(M_{\text {gas,tot }}\right)$. Note that the initial baryon fraction $f_{\mathrm{b}} \approx M_{\mathrm{gas}, \mathrm{s}} / M_{\mathrm{s}} \approx M_{\mathrm{gas}, \mathrm{vir}} / M_{\mathrm{vir}} \approx 10 \%$ and the radius containing half the initial gas within $r_{\mathrm{s}}$ is $r_{0.5 \mathrm{~g}}$.

We consider both clumpy and smooth gas distributions within the low-mass halos. For the clumpy models, we make the important assumption that the confined gas has a fractal distribution before the SN explodes. There are two reasons for this assumption. First, the infalling gas becomes shocked and 


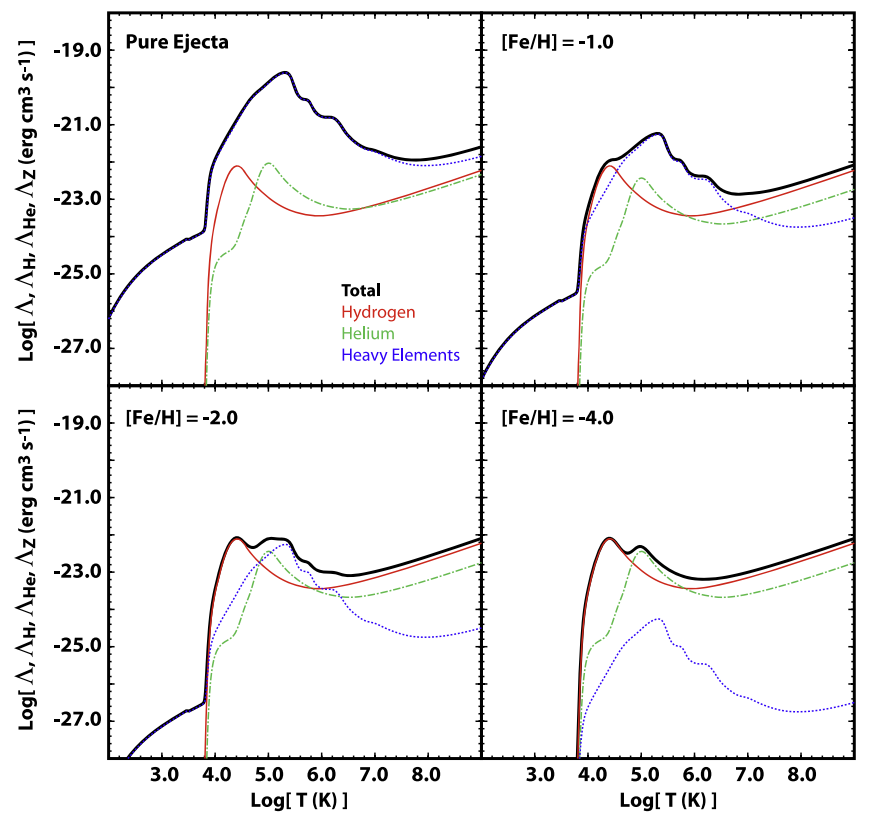

Figure 3. Variable cooling function, using compositions encountered in the simulations. In each panel, the thick line is the total cooling, the thin red line is cooling due to hydrogen, the green dot-dashed line is due to helium, and the metals combined produce the blue dotted line. In the single SN model, the internal metal ratios are fixed by the $\mathrm{SN}$ composition and are grouped into a single function.

highly inhomogeneous close to the dark halo core (Abel et al. 2000). Second, a high-mass star that is destined to become an $\mathrm{SN}$ injects mechanical and ionizing energy into the surrounding gas prior to the explosion. This energy input drives turbulence in the surrounding gas (Elmegreen \& Scalo 2004).

In looking for possible key factors in the retention, we consider the following scenarios.

1. Abundances and cooling: We use chemical abundances that approximate primordial conditions starting at $[\mathrm{Fe} / \mathrm{H}]=-4.0$. This starting metallicity is conservative: Wise \& Abel (2008) show that the initial metallicities of the first galaxies could have been as elevated as $[\mathrm{Fe} / \mathrm{H}] \sim$ -3.0 or higher. We compute models with cooling, and models $\left(\gamma=\frac{5}{3}\right)$ where we have artificially turned off microphysics affecting energy loss (e.g., cooling through metal and recombination lines). The cooling function is presented in Figure 3; the abundances are given in Appendix A.

2. Clumpiness: We consider models with quasi-fractal cool star-forming gas, and comparison models where the gas is homogeneous and smooth. The key point here is that the degree of coupling between the SN energy and the surrounding medium will depend on whether the medium is smooth or structured.

3. Central and off-center explosions: We consider a single $10^{51}$ erg SN explosion, located either at the geometrical center of the halo potential or off-center at a radius that encloses half the mass contained inside one scale radius of the potential. SNe that occur away from the center have an asymmetric environment.

This gives, in combination, eight scenarios for each of the halo masses considered. In each case, the mass and energy contained in the inner scale radius are integrated at $25 \mathrm{kyr}$ intervals and plotted over the course of the simulation to obtain estimates for the masses retained at late times.

In the Fyris Alpha models presented here, we use a fixed dark matter potential that dominates everywhere. Future models will include self-gravity in the gas. Therefore, we limit the initial baryonic mass inside a scale radius $\left(r_{\mathrm{s}}\right)$ to be $10 \%$ of the dark matter mass inside that radius. This results in an overall baryonic fraction out to the virial radius $\left(r_{\mathrm{vir}}\right)$ of $f_{\mathrm{b}} \approx$ $12 \%$, less than the canonical $f_{\text {crit }} \approx 17 \%$ global baryonic mass fraction (Planck Collaboration et al. 2014). Our choice of $f_{\mathrm{b}}$ is a conservative assumption because a lower gas budget generally assists the $\mathrm{SN}$ in accelerating and heating the gas. If the models retain gas with these lower mass fractions, they can be reasonably expected to do so with more gas in place.

\subsection{Dark Matter Halo}

In recent years, high-resolution cold dark matter (CDM) models have identified the need for a halo density profile that has an additional parameter beyond those used in the NavarroFrenk-White (NFW) profile (Hayashi et al. 2004; Springel et al. 2008). The profiles seen in the latest simulations display local density derivatives that are power laws of radius, unlike the NFW profile that has asymptotic behavior at small radius. They invoke a generalized exponential function that has this property and note that it has been used in the past by Einasto (1965) in the context of galactic halo spatial denities. ${ }^{6}$

We use the Einasto function, a generalized exponential, to form a dimensionless potential defined by the parameters $\alpha$, a halo concentration factor $x_{\text {vir }}$ (often referred to as $c$ ), and the density contrast of the halo $\Delta(\approx 178)$ with respect to the universal background (Nichols \& Bland-Hawthorn 2009). We stress that these parameters must be specified at a fixed redshift $z$ because of their strong cosmic evolution (Power et al. 2003; Duffy et al. 2008).

We consider four halo mass models (M55, M60, M65, M70) defined in Section 2.2. The total halo masses are (3.0, 2.7, 2.5, 2.3 ) times the virial mass, respectively (e.g., Figure 4). The halo and gas properties are summarized in Table 3. Our normalization uses a density function value of $1 / e$ at the scale radius, $r_{\mathrm{s}}$. This gives a slightly different set of scaling constants, but recovers exactly the same physical quantities as the 1/4 scaling used in Nichols \& Bland-Hawthorn (2009). This change was motivated by wanting to compare with dimensionless isothermal potentials used by Sutherland \& Bicknell (2007), which also obtained a scaled density value of $1 / e$ at the scale radius. (We refrain from using the popular "core" radius because, unlike the isothermal potentials, the generalized Einasto functions do not have a distinct core region; here we use $r_{\mathrm{s}}$ throughout.)

Our adopted halo parameters are based on those of Duffy et al. (2008) and Springel et al. (2008). As their fits to the simulated halos are measured over a relatively low redshift range, we extrapolate these properties back to a redshift of $z=10$. At the time of writing, we became aware of new work (Correa et al. 2015) where the parameters are extended back to $z=20$, but these appear to be consistent with our estimated values, particularly for the important M65 and M70 models.

\footnotetext{
6 This has the same functional form as the projected Sersic profile, but the 2D function does not transform to the same underlying radial density distribution.
} 

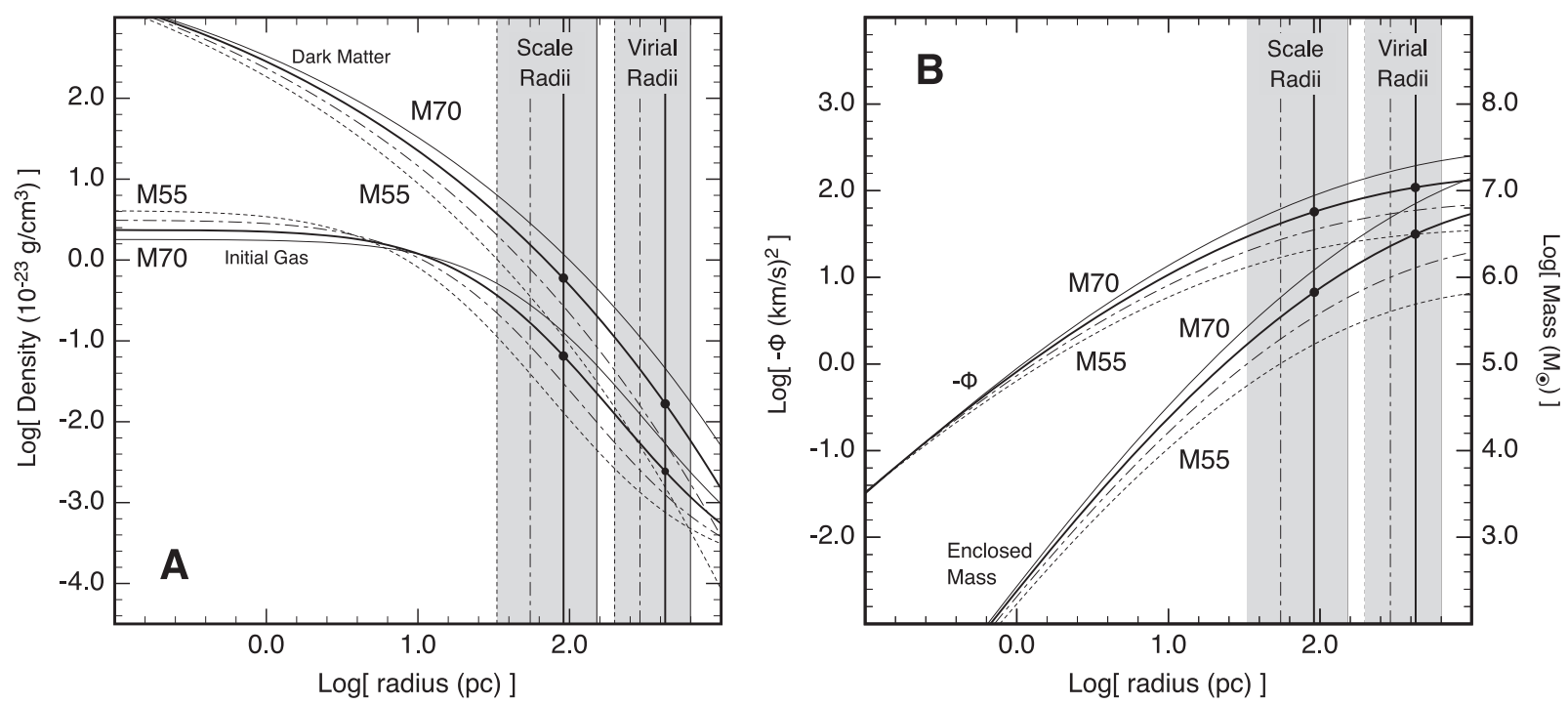

Figure 4. (A) Dark matter and initial gas density (neutral gas, $\mu=1.21, \beta=2.0)$ profiles for each of the model halos. (B) Potential functions $(-\Phi)$ and enclosed dark matter masses. In both panels, the dashed, dot-dashed, thick solid, and thin solid curves are for the M55, M60, M65, and M70 models, respectively. The vertical gray bands cover the radial extent of four scale radii $\left(r_{\mathrm{s}}\right)$ and four virial radii $\left(r_{\mathrm{vir}}\right)$, marked with four vertical lines, for the four mass models using the same line type for each model.

Table 3

Naming Convention for All Numerical Simulations

\begin{tabular}{|c|c|c|c|c|c|c|c|c|}
\hline \multicolumn{9}{|c|}{ Models without Preionization } \\
\hline \multirow[b]{3}{*}{ Halo Mass } & \multicolumn{4}{|c|}{ Central Explosions } & \multicolumn{4}{|c|}{ Off-center Explosions } \\
\hline & \multicolumn{2}{|c|}{ Smooth } & \multicolumn{2}{|c|}{ Clumpy } & \multicolumn{2}{|c|}{ Smooth } & \multicolumn{2}{|c|}{ Clumpy } \\
\hline & Adiabatic & $\overline{\text { Cooling }}$ & Adiabatic & Cooling & Adiabatic & $\overline{\text { Cooling }}$ & Adiabatic & $\overline{\text { Cooling }}$ \\
\hline $10^{5.5} M_{\odot}$ & M55CSA & M55CSC & M55CCA & M55CCC & M55OSA & M55OSC & M55OCA & M55OCC \\
\hline $10^{6} M_{\odot}$ & M60CSA & M60CSC & M60CCA & M60CCC & M60OSA & M60OSC & M600CA & M600CC \\
\hline $10^{6.5} M_{\odot}$ & M65CSA & M65CSC & M65CCA & M65CCC & M65OSA & M65OSC & M65OCA & M65OCC \\
\hline $10^{7} M_{\odot}$ & $\cdots$ & $\ldots$ & $\ldots$ & $\cdots$ & $\cdots$ & $\cdots$ & $\cdots$ & $\cdots$ \\
\hline
\end{tabular}

Models with Preionization

\begin{tabular}{|c|c|c|c|c|c|c|c|c|}
\hline \multirow[b]{3}{*}{ Halo Mass } & \multicolumn{4}{|c|}{ Central Explosions } & \multicolumn{4}{|c|}{ Off-center Explosions } \\
\hline & \multicolumn{2}{|c|}{ Smooth } & \multicolumn{2}{|c|}{ Clumpy } & \multicolumn{2}{|c|}{ Smooth } & \multicolumn{2}{|c|}{ Clumpy } \\
\hline & Adiabatic & Cooling & Adiabatic & Cooling & Adiabatic & Cooling & Adiabatic & Cooling \\
\hline $10^{5.5} M_{\odot}$ & $\cdots$ & $\cdots$ & $\cdots$ & $\cdots$ & $\cdots$ & $\cdots$ & $\cdots$ & $\cdots$ \\
\hline $10^{6} M_{\odot}$ & $\cdots$ & $\cdots$ & $\cdots$ & $\mathrm{M} 60 \mathrm{CCH}$ & $\cdots$ & $\cdots$ & $\cdots$ & M60OCH \\
\hline $10^{6.5} M_{\odot}$ & $\ldots$ & $\cdots$ & $\cdots$ & $\mathrm{M} 65 \mathrm{CCH}$ & $\cdots$ & $\ldots$ & $\cdots$ & M65OCH \\
\hline $10^{7} M_{\odot}$ & $\cdots$ & $\ldots$ & $\ldots$ & $\mathrm{M} 70 \mathrm{CCH}$ & $\cdots$ & $\cdots$ & $\cdots$ & M70OCH \\
\hline
\end{tabular}

\subsection{The Cold Interstellar Medium}

In our simulations, we introduce a non-uniform medium to study the influence of inhomogeneity on the dynamical interaction between the SN ejecta and the surrounding gas. To assess the importance of inhomogeneity, we run both homogeneous (smooth) and inhomogeneous (clumpy) models and compare the outcomes. Following Sutherland \& Bicknell (2007), to establish a non-uniform medium, we make use of an analogy with a turbulent medium. Rather than attempt to review this huge topic, we refer the reader to astrophysically oriented reviews as a starting point for background material (Elmegreen \& Scalo 2004; Scalo \& Elmegreen 2004).

We represent the non-uniform properties of the turbulent medium using three statistical characteristics: the variance, $\sigma_{F}^{2}$, the intermittency (by using lognormal distributions), and a selfsimilar power-law structure. We achieve both the property of intermittency and power-law structure simultaneously with an iterative scheme first developed by Lewis \& Austin (2002); see Appendix B for more details. Hence, the initial distribution of the ISM that we employ should be regarded as a physically motivated generalization of a homogeneous model.

We further simplify our turbulent medium by neglecting the velocity structure, and we focus on choosing statistical parameters to describe the density alone. This is justified numerically by the relatively small turbulent velocities expected when compared to the very large velocities found in our global SN-ISM interaction. The velocities observed in typical warm ISM conditions fall in a range of transonic to 


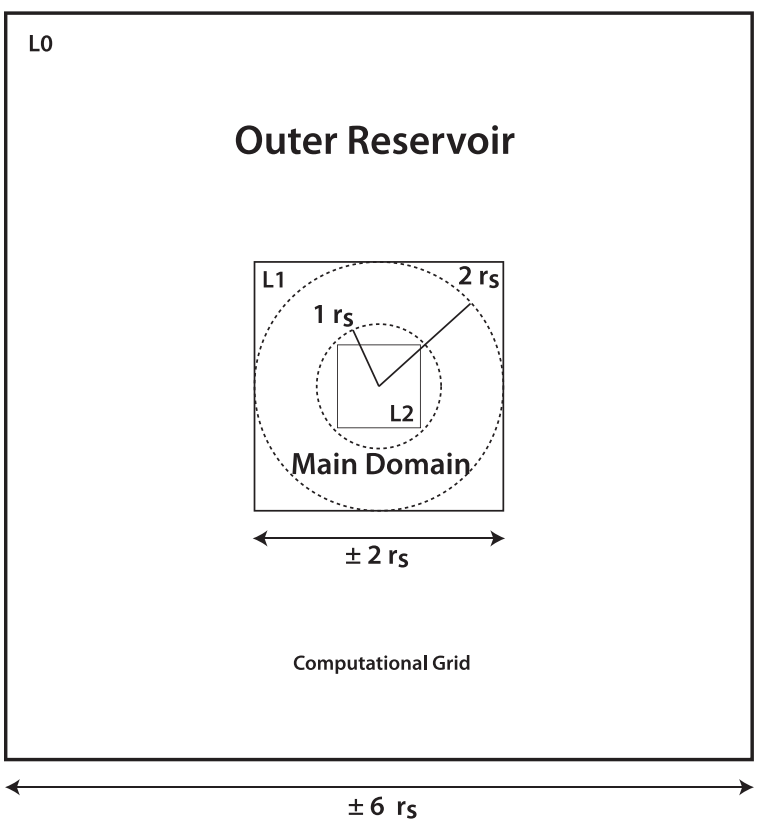

Figure 5. 2D representation of the $3 \mathrm{D}$ computational domain. Each square represents a cubic domain enclosing the interior regions. The main level of interest is L1, where the mass and energy transport during the explosions are measured. A third inner level, L2, is used to resolve early-epoch $(t<50 \mathrm{kyr})$ thin-shell phases of the SN remnant.

mildly supersonic values, Mach 1-5 (e.g., Heiles et al. 2004). At temperatures at or below $10^{3} \mathrm{~K}$, this corresponds to velocities $\ll 4 \mathrm{~km} \mathrm{~s}^{-1}$. In our $0.1-1 \mathrm{kpc}$ simulations over $10^{7} \mathrm{yr}$ timescales, the resulting displacements amount to only a few cells at most and are insignificant compared to those of the energy bubble generated by the SN outburst, where velocities of many hundreds of kilometers per second occur. Consequently, we do not impose a turbulent velocity field on the cold ISM medium.

\subsection{Hydrodynamic Calculations}

The modeling was carried out using the Fyris Alpha code (Bland-Hawthorn et al. 2007; Sutherland 2010). It solves ideal Euler hydrodynamic flows of gas with cooling and an equation of state appropriate to astrophysical gases, in the presence of a fixed gravitational field. The code uses a third-order semiLagrangian shock-capturing method that is robust and well suited to the extreme temperatures and Mach numbers encountered here.

The computational domain consists of a three-dimensional nested series of cubic meshes, as illustrated by the schematic in Figure 5. Each level is a factor of 3 smaller in physical domain and higher resolution than its outer containing level, covering the central third of the enclosing level. Each level is $216^{3}$ cells with an overall effective resolution of $1944^{3}$ cells.

A relatively low resolution outer level, L0, covers the halo out to \pm 6 scale radii and provides an outer reservoir and boundary condition for the main level of interest, L1. The outer reservoir reduces or avoids completely any boundary artifacts on L1 due to gravity bringing new gas onto the main level when infall conditions apply, keeping level L1 as physically consistent as possible at all times. L0 contains the virial radius. Velocities at the outer boundary are extremely small at all times considered here, and the outer LO boundary is essentially fixed.
The main level, L1, covers a region of just over \pm 2 scale radii and is the primary region where the movement and evolution of mass and energy are measured. Early epochs require higher resolution to resolve thin spherical shell radiative blast waves, so a third, inner injection level, L2, is added to resolve the early phases of the SNe. After $25 \mathrm{kyr}$, L2 is disabled and the remainder of the simulations are carried out on L0 and L1.

For each halo mass, the scale radius varies, and so the resolution is variable. The highest resolution, on level L2, is $\Delta x \sim 0.25,0.37$, and $0.62 \mathrm{pc}$ per cell for the M55, M60, and M65 models, respectively. Thus, the effective mass resolution is $\sim 10^{4} M_{\odot} / 216^{3}$, or of order $10^{-3} M_{\odot}$; in practice, cell densities can vary by more than 4 orders of magnitude across the grid.

The remaining key features of the calculations can be summarized as follows.

1. A fixed gravity potential, interpolated locally by thirdorder PPM interpolation to match the interpolation of the hydrodynamical algorithm, taking conservative potential differences to compute mean accelerations.

2. SNe simulated by depositing $10^{51}$ erg of energy as internal gas energy in a smoothed spherical region, as small as possible while retaining spherical geometry well enough to prevent gross non-spherical "pixel" errors, typically 6 cell radius on L2, corresponding to $1.5-3.7 \mathrm{pc}$. This then takes the form of a pulse of low-density, hot overpressure gas, which then expands, converting internal energy to kinetic energy and doing work against the potential. Energy is also lost via cooling.

3. To allow for significant changes in the local metallicity in the models as the metal-rich ejecta propagate through the models, a generalized cooling model has been adopted that allows the local composition of each cell to be determined during the simulation. Instead of a single cooling function and uniform molecular weight, the cooling was separated into three components, representing hydrogen, helium, and heavier metals, and ionization was taken into account.

The metal-rich SN ejecta were followed using a scalar tracer variable, advected passively, representing the SN material, and hence the local helium fraction and metal fraction. The local enrichment of the gas was thus computed at each time step. Furthermore, the ionization fraction of the components, precomputed with the MAPPINGS IV code (Sutherland et al. 1993; Allen et al. 2008) as a function of temperature, allowed the equation of state to vary with the mean molecular weight, ensuring more accurate shock temperatures, and allowing for a wider range of cooling timescales caused by strongly varying composition. Figure 3 shows the resulting computed cooling functions for a range of encountered compositions on the grids.

We acknowledge that there are large uncertainties in the yields from essentially zero metallicity stellar SN models. The yield of metals, particularly oxygen, could have an impact on the outcome, lower-mass SNe giving less enrichment and cooling, while higher-mass stars could give more cooling. More detailed future work incorporating a range of $\mathrm{SN}$ progenitor masses will investigate the impact of different SN assumptions. By allowing for the ionization and composition to vary from cell to cell, we obtain more accurate species columns, for example, for neutral and ionized hydrogen. 
Table 4

Thermalization Efficiency in the M60 and M65 Models

\begin{tabular}{|c|c|c|c|c|c|c|c|c|}
\hline \multirow{2}{*}{$\begin{array}{r}\text { Model } \\
\text { Time }\end{array}$} & \multicolumn{4}{|c|}{ Central Explosions } & \multicolumn{4}{|c|}{ Off-center Explosions } \\
\hline & Adiabatic & $\overline{\text { Cooling }}$ & Adiabatic & $\overline{\text { Cooling }}$ & $\overline{\text { Adiabatic }}$ & $\overline{\text { Cooling }}$ & Adiabatic & $\overline{\text { Cooling }}$ \\
\hline M60 & $100 \%$ & $11 \%$ & $100 \%$ & $15 \%$ & $100 \%$ & $11 \%$ & $100 \%$ & $16 \%$ \\
\hline
\end{tabular}

Our focus is on the hotter atomic and ionized phases of highpressure gas and the cooling therein, as that gas is volume filling and will have the primary effect on the global dynamic evolution if any. Gas in the molecular phase is likely to be confined to the centers of the densest cores, if present at all. As the outflows are all warm $\left(>10^{4} \mathrm{~K}\right)$, they are expected to be atomic or ionized in any case. In the face of uncertainty in the formation of $\mathrm{H}_{2}$ under primordial conditions and little or no information on $\mathrm{CO}$ cooling, we leave molecular cooling out. It would be essential to include it if we were tracking the (much longer) time required for the hot gas to re-cool back to starforming temperatures $\ll 100 \mathrm{~K}$, but here we are focused on the initial halo gas retention problem. We defer the star formation problem to more substantial investigations.

That we neglect molecular cooling in these simulations can be considered a conservative approximation, but we expect it to have little effect on the global dynamics of gas retention. More than $99.9 \%$ of the internal energy is lost by the time the gas cools back below $10^{4} \mathrm{~K}$ from early temperatures of more than $10^{7} \mathrm{~K}$, so only a tiny fraction of the SN energy is available to the molecular cooling in the present simulations.

In Table 4, we calculated the thermalization efficiency for a subset of the models as a check that our simulations correctly handle cooling. The thermalization efficiency was estimated by finding the ratio of the total energy at the time when gas is first leaving the grid to the total initial energy. Our efficiencies should be considered upper bounds, as it is likely that some cooling will occur at later times. From Thornton et al. (1998), we expect the thermalization efficiency to be $\sim 10 \%$, which is consistent with the results of our models.

\subsection{Preionization from the SN Progenitor}

The preionization of the gas by the SN precursor plays a key role in the loss or survival of gas after the SN explosion, particularly in low-mass dark matter halos. We consider in detail the impact of an ionized $\mathrm{H}_{\mathrm{II}}$ region that forms around the progenitor star prior to the explosion. The key parameters of this model are the stellar properties $\left(T_{\mathrm{eff}}, L\right)$ and the associated stellar atmospheres. These give the ionizing photon flux as a function of time up to the SN event. Transforming the grid coordinates to spherical coordinates around the star, radial summations to approximate the optical depth integrals are performed, resulting in thermal and ionization structures calculated from the MAPPINGS IV ionization code. These are then mapped back to Cartesian coordinates and used in the Fyris fluid computations. It was necessary to perform this remapping for every time step in the fluid dynamics simulation in order to track the evolution in opacity. This slowed the code by a factor of 5-10. We considered both clumpy and smooth external media with and without gas cooling.

We model the SN progenitor with a low-metallicity star of $25 M_{\odot}$. Higher-mass stars produce stronger ionizing intensity and therefore assist gas loss by overheating the gas prior to the SN explosion. But since $80 \%$ of all SN events in a conventional IMF have lower mass and therefore weaker ionizing radiation fields, we consider our choice of the upper mass limit to be conservative. We use the evolutionary tracks of Meynet \& Maeder (2002, hereafter MM02) for a rapidly rotating, lowmetallicity massive star. The $25 M_{\odot}$ track was missing from that work, and so we have interpolated the main-sequence phase of this model from the other stellar models. In Figure 6(A), we show the interpolated values of the luminosity and temperature for the $25 M_{\odot}$ model at $[\mathrm{Fe} / \mathrm{H}] \sim-3.7$ (MM02). These are the closest published tracks for our required starting metallicity of $[\mathrm{Fe} / \mathrm{H}]=-4.0$. We used the tabulated MS lifetime of 6.1 Myr; the interpolated temperatureluminosity track is shown in Figure 6(B).

In Figure 7, we show the impact of the total ionizing budget during the pre-SN phase for our adopted stellar model and a perfect blackbody of the same surface temperature. The panels $\mathrm{A}, \mathrm{C}$, and $\mathrm{E}$ refer to the models with the progenitor at the center; panels B, D, and F are for the off-centered models. This gives some indication of the sensitivity to the stellar models. If the thin lines exceed the thick lines, then a Strömgren sphere is possible within the radius, and the stellar radiation would be incapable of extending beyond that, which is the initial case for all halos. However, as the gas responds to heating and evolves, the density is smoothed and falls on average owing to outflows. The quadratic dependence of recombinations means that the available recombinations falls rapidly, and once the photon curves exceed the recombination curves, the photoionized zones can grow rapidly and ionize more of the halo.

In the upper panels, the M60 halo will be easily fully ionized after the first $2 \mathrm{Myr}$. The middle panels show that the M65 models are marginal if the photons are uniformly spread throughout the halo, and capable of ionizing the inner core region and possibly beyond before the SN occurs. The actual result will depend on the details of the radiative transfer, whereas this is an indicative integral view of the processes that does not take the geometry of the ionized volume into account. The lower panels for M70 indicate that the stars are insufficient to photoionize a significant volume of the halo.

These indications are broadly supported by the simulations: M60 is rapidly ionized, M65 is able to break out of the core radius before the $\mathrm{SNe}$, and the ionized region is contained in the M70 models.

Stellar winds. To avoid the complexities of rapid post-mainsequence evolution, we assume that the star simply explodes as an SN at the end of the main sequence. Ekström et al. (2006) showed that mass loss during the main sequence in similar stars at very low metallicity may only amount to $1 \%$ of the initial mass, and Kudritzki $(2002,2005)$ showed that the stellar wind luminosities of low-metallicity $([\mathrm{Fe} / \mathrm{H}] \leqslant-4.0) \mathrm{O}$ stars are typically $10^{34} \mathrm{erg} \mathrm{s}^{-1}$ or less, several magnitudes below an equivalent solar-metallicity star. We combine these values to 

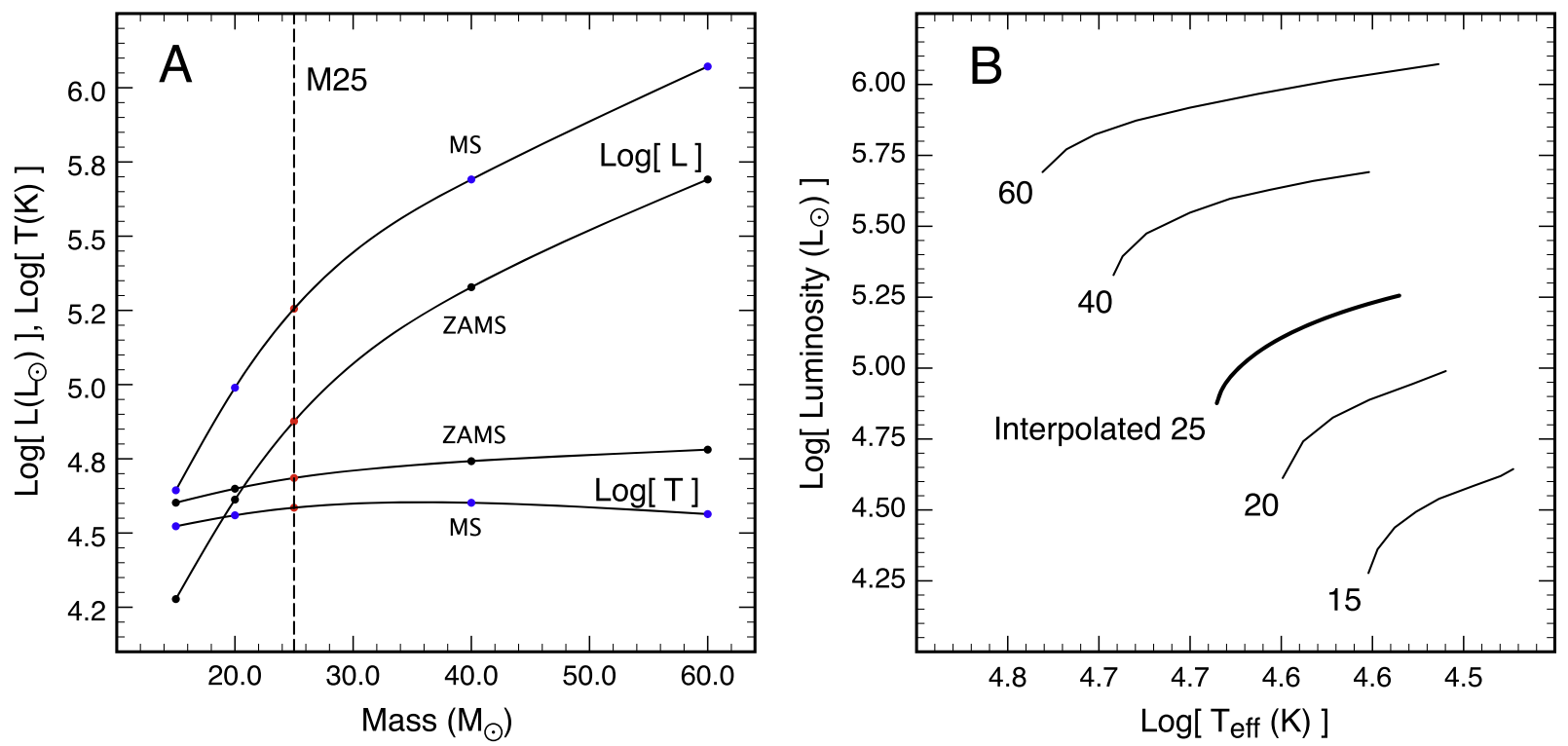

Figure 6. (A) Interpolated luminosity and temperature for the $25 M_{\odot}$ model in the MM02 grid. The zero-age main sequence is labeled ZAMS and the end of the main sequence as MS. (B) Evolutionary tracks for the four most massive rotating $\left(300 \mathrm{~km} \mathrm{~s}^{-1}\right)$ star models in MM02 at $[\mathrm{Fe} / \mathrm{H}] \approx-4$. We adopt a $25 M_{\odot}$ star model interpolated from these tracks with cubic splines using the endpoints in (A). For this star, the time span from the ZAMS to the explosion is 6.1 Myr.

get a mean wind velocity, for a main-sequence mass loss of $1 \%$ :

$$
v_{w} \sim 880\left[\frac{L_{w}}{10^{34} \mathrm{erg} \mathrm{s}^{-1}}\right]^{1 / 2}\left[\frac{M}{25 M_{\odot}}\right]^{-1 / 2}\left[\frac{t_{\mathrm{MS}}}{6.1 \mathrm{Myr}}\right]^{-1 / 2} \mathrm{~km} \mathrm{~s}^{-1} .
$$

This wind is included in the pre-SN evolution, but the small mass flux and low ram pressure meant that it had negligible effect on the simulations. Once the $\mathrm{H}$ in region pressurized the surrounding region, the stellar wind was unable to blow a wind bubble of any significant size.

The key parameter for these near-zero-metallicity $\mathrm{O}$ stars is their unusually high effective temperatures for a given mass. At $25 M_{\odot}$, we estimate the initial temperature to be $48,000 \mathrm{~K}$, compared to a similar-mass star at solar metallicity of only 40,000 K (see, e.g., Meynet \& Maeder 2000 and more recently Georgy et al. 2012). This means that the star will be more effective as a source of ionizing photons than the equivalent star in the solar neighborhood. To convert the evolutionary tracks, $T_{\text {eff }}(t)$ and $L(t)$, to an ionizing photon-luminosity (see Figure 8), we used the ATLAS9 atmospheric grid (Castelli \& Kurucz 2004), which uniquely contains a set of atmospheres for $[\mathrm{Fe} / \mathrm{H}]=-4.0$ with alpha-element abundance enhancements, perfectly matching the initial gas composition in our simulations. The interpolated stellar temperature, luminosity, and ionizing fluxes are shown in Figure 8. The derived ionizing spectrum is shown in Figure 9; for comparison, a photon flux assuming a blackbody instead of the stellar atmosphere is included.

\section{SIMULATIONS}

In the absence of considering the full initial value problem (i.e., infall, self-gravity), we assume that sufficient gas has already settled onto a spherical dark matter halo, and that it has dissipated energy and come to rough dynamical equilibrium. Fundamentally, an isothermal hydrostatic equilibrium in a gravitational potential, $\Phi$, is only possible with a uniform medium, with the requirement that the local pressure gradients oppose the local potential gradient, that is,

$$
\frac{1}{\rho} \frac{d P}{d r}=-\frac{d \Phi}{d r}
$$

where $P$ and $\rho$ are the gas pressure and density at a radius $r$. As the potential is smooth, the pressure gradients must be well behaved. When the density is smooth also, a solution for a given temperature is possible, with the temperature defining the pressure scale height. However, when the density is no longer locally smooth, corresponding local temperature variations are required to give the well-behaved pressure gradients needed for hydrostatic equilibrium. Without a single global temperature, a range of scale heights exist over the whole domain, and a single structure scale - and hence equilibrium - is not strictly possible. However, in practice it is close to equilibrium, and the timescales for change are much longer than those that describe the star formation in our models.

When gas falls into a gravitating potential well, it undergoes complex motions as it settles toward dynamical equilibrium. Some of the kinetic and potential energy is likely to be converted to thermal energy, which is then lost through radiative cooling. If there were no losses, after adiabatically virializing in the halo potential, the gaseous medium would have an initial temperature, $T_{\text {init }}$, related to the virial temperature of the halo such that $T_{\text {init }} \sim T_{\text {vir }}$. For the adiabatic case, $T_{\mathrm{vir}}=\mu m_{\mathrm{H}} G M_{\mathrm{vir}} / k r_{\mathrm{vir}}$, for which $k$ is Boltzmann's constant, $\mu$ is the mean molecular weight of the gas, and $m_{\mathrm{H}}$ is the mass of the $\mathrm{H}$ atom. This is before any star formation takes place.

We now introduce the common $\beta$ notation used in the presence of complex multiphase gases (Cavaliere \& FuscoFemiano 1976; Arnaud 2009). An initial (quasi-)hydrostatic gas distribution depends on an effective temperature $T_{\text {eff }}=T_{\text {gas }}+T_{\text {turb }} \sim T_{\text {init }}$ arising from a combination of thermal and turbulent support (plus other terms due to magnetic 


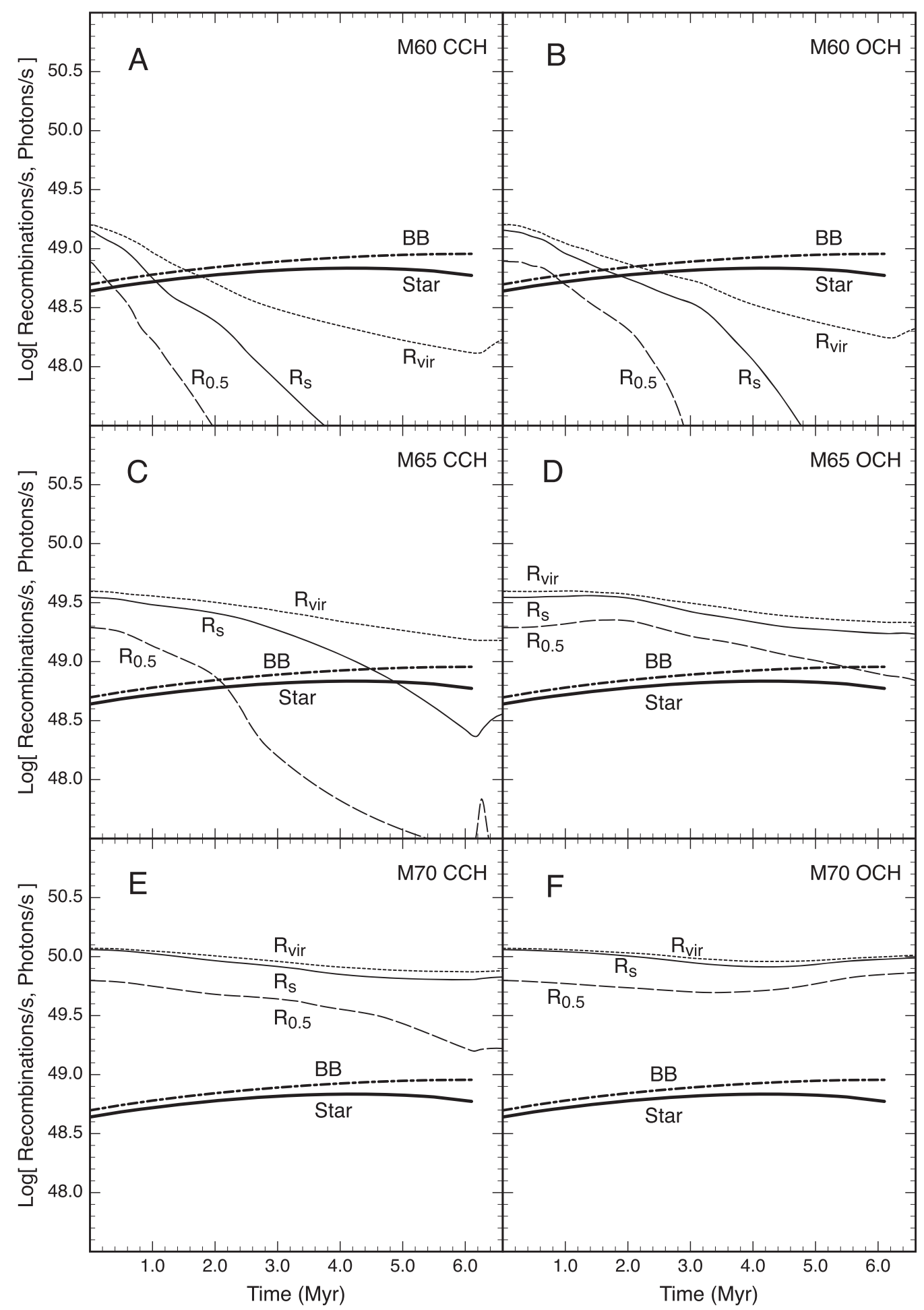

Figure 7. Comparison of the instantaneous photon rate (thick lines) compared to the total available recombinations as the models progress in the pre-SN phase. The left-hand panels (A, C, E) correspond to the centered models; the right-hand panels (B, D, F) refer to the off-centered models. The two thick lines represent a pure blackbody model (BB) and an extreme low-metallicity stellar atmosphere (Star), and the difference between them gives an indication of the magnitude of any uncertainties in the atmosphere models. Available recombinations are computed by integrating $n_{e} n_{\mathrm{H}}^{+} \alpha\left(T_{4}\right)$ in $\mathrm{cm}^{-3} \mathrm{~s}^{-2}$ over the respective volumes, where $\alpha$ is the recombination coefficient summed over all possible transitions and $T_{4}$ is the gas temperature in units of $10^{4} \mathrm{~K}$. This yields the total number of recombinations per second if all of the gas can be ionized, ignoring optical depth and geometry of the ionized volume.

fields that we neglect at present). With radiative losses, the initial thermal pressure is lost, and we are left with turbulent support only. Thus, if we assume initial equipartitition between thermal and turbulent pressure, $T_{\text {eff }}=T_{\text {turb }} \approx 0.5 T_{\text {vir }}$ such that $\beta=T_{\mathrm{vir}} / T_{\mathrm{eff}}=2.0$.
For all of our models, we adopt a $\beta=2.0$ gas distribution to recognize that the baryons must undergo some cooling before the first stars have formed. It is conceivable that $\beta$ is larger, but such an assumption would concentrate the gas closer to the center of the potential, thereby assisting gas retention. Indeed, 


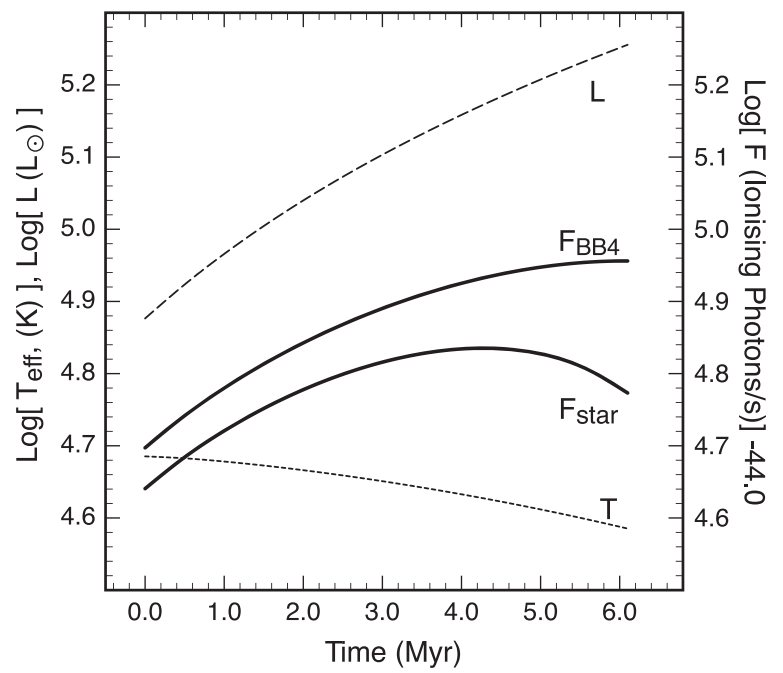

Figure 8. Interpolated $L(t)$ (long-dashed line), $T_{\text {eff }}(t)$ (short-dashed line), and ionizing photon fluxes (thick lines) for M25. $F_{\text {star }}$ assumes stellar atmosphere models, and a comparison is shown, $F_{\mathrm{BB}}$, which is the flux assuming a simple blackbody approximation to the stellar spectrum. The star emits between $5 \times 10^{48}$ and $7 \times 10^{48}$ ionizing photons s ${ }^{-1}$ over its lifetime.

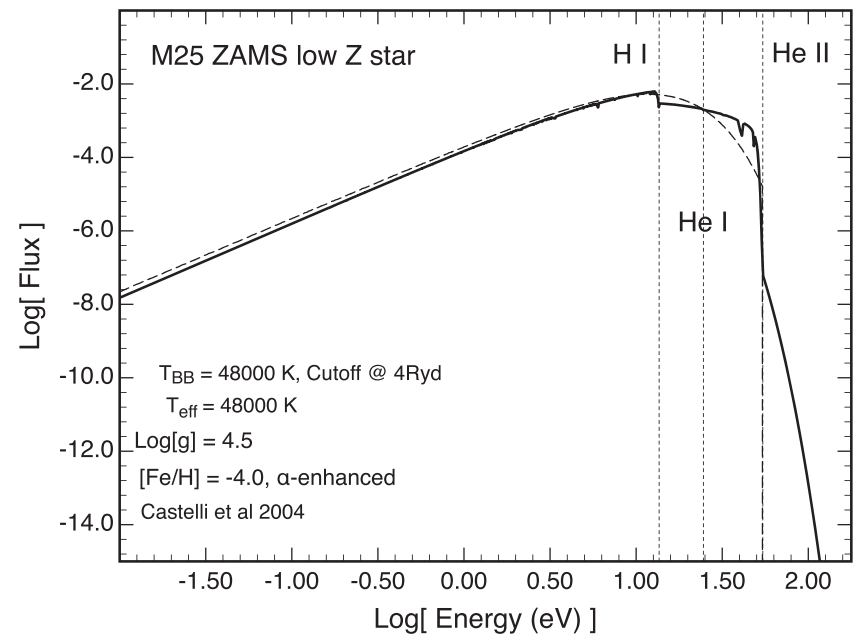

Figure 9. Stellar spectrum (solid line) of our adopted $25 M_{\odot}$ star model using an ATLAS9 ZAMS stellar atmosphere at $[\mathrm{Fe} / \mathrm{H}] \approx-4$ (Castelli \& Kurucz 2004). The dashed line is the blackbody curve for the same surface temperature $T_{\text {eff }}=48,000 \mathrm{~K}$.

Wise et al. (2008) find that baryonic matter is more concentrated than the dark matter in their evolving halos $\left(M_{\text {tot }}=10^{8} M_{\odot}\right)$. These authors use an adaptive mesh code to track the development of supersonic turbulence driven by accretion over many orders of magnitude in physical scale. In combination with line cooling, the supersonic turbulence leads to central densities that are several orders of magnitude higher than what we can achieve with Fyris.

While we have the option to modify $\beta$ to increase the central gas densities, this would only assist our ability to retain gas after the SN. Our adoption of a lower constant $\beta$ value has the benefit of reducing any central concentration differences in the models, leaving just the global gravity potential as the key mass model difference. The resulting temperature distributions for the M55-M70 models are discussed below.

Initial state of the gas. We adopt a mean equilibrium distribution for the gas density given by $\rho=\rho(0) \exp (\Phi)^{\beta}$, for

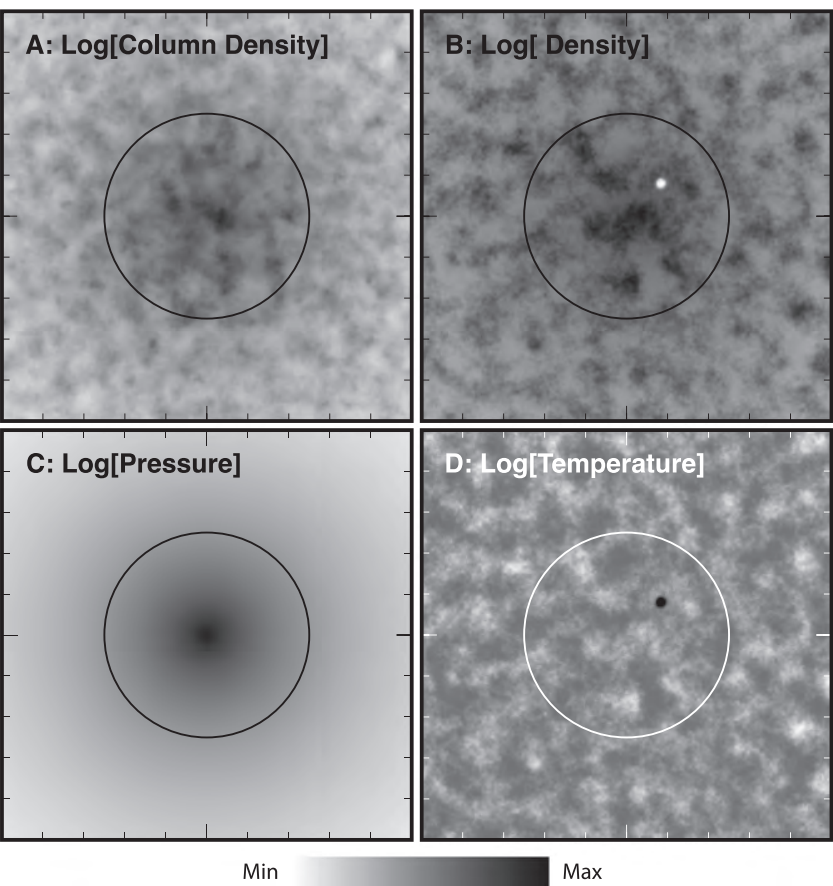

Figure 10. Initial distributions of density, pressure, and temperature (level L01) for the M60 off-centered SN model; the distributions are similar for M55, M65, and M70. While the density and temperature structure are fractal, the overall pressure is constant at the start. Each panel shows a slice through the middle of the halo, and the quantity shown is scaled logarithmically; the circle has a radius of $r_{\mathrm{s}}$, the scale radius. (A) Relative column density over the range -4.0 (white) to 0.0 (black). (B) Relative density over the range -5.0 (white) to 0.0 (black). The off-centered SN is visible as a white dot. (C) Relative pressure ranging from -5.0 (white) to 0.0 (black). (D) Temperature ranging from 1.0 (white) to $>4.0$ (black)

which $\Phi$ describes the dark halo potential (Figure 4, right panel). This is described in detail in Appendix B. The use of $\beta$ allows for the gas scale length to be different from $r_{\text {vir }}$ (Figure 4, left panel). For completeness, note that the dynamical dispersion of the dark halo is $\sigma=\beta c_{\mathrm{s}}$. For a given temperature and composition/ionization state, $P \propto \rho$. So for homogeneous models, there is a uniform temperature everywhere such that the local density $\rho$ is equal to the mean density $(\rho /\langle\rho\rangle=1.0)$. For fractal models, $\rho /\langle\rho\rangle=f(x, y, z)$, for which $f$ is the normalized fractal, giving a distribution $T \propto P / \rho$ that is the same for all the models using the same fractal modulation. The distribution $f$ has a mean value of unity and a variance of 5 (see Appendix B).

The projected 2D gas distribution (density, pressure, temperature) for the inhomogeneous M60 model is shown in Figure 10. The temperature histogram for Figure 10(D), and for all models, is given in Figure 11. Across the models, the global mean gas temperature spans the range 500-2000 K, with a small gas fraction extending below a few hundred kelvin, compatible with a star-forming region. The bulk of the ISM tops out at about $10^{4} \mathrm{~K}$ and thus covers the range of temperatures seen in typical warm and cold neutral ISM media (Sternberg et al. 2002).

To verify the equilibrium of the initial conditions, we ran adiabatic test models with smooth gas in hydrostatic equilibrium, and the initial distribution remained steady for $\gtrsim 200$ Myr, much longer than the $25 \mathrm{Myr}$ timescale of our SN model. An adiabatic clumpy test model could not achieve perfect equilibrium and evolved toward the smooth solution, smearing 


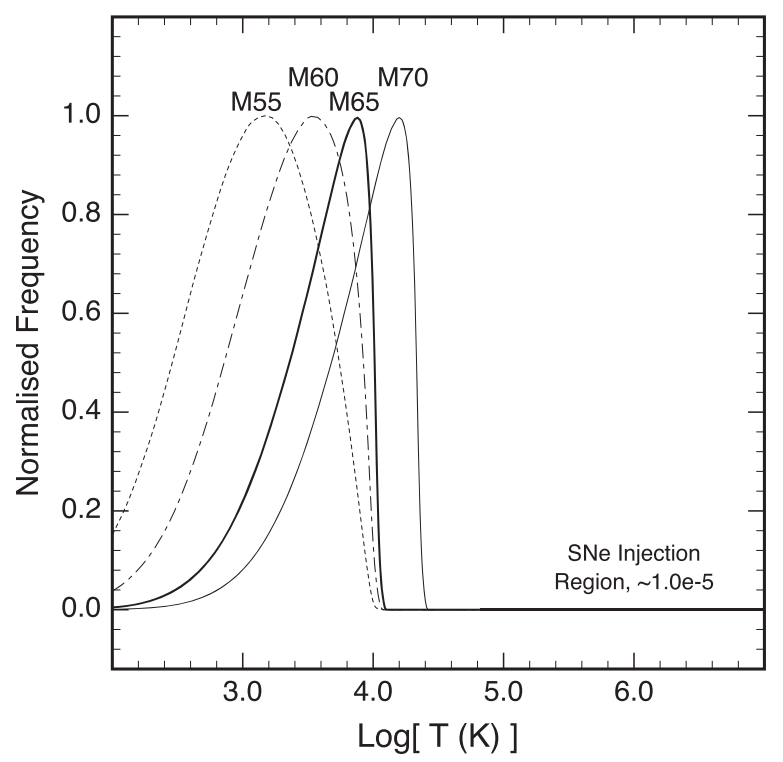

Figure 11. Initial gas temperature distribution for the material inside one scale radius, $r_{\mathrm{s}}$, for the models with fractal clumpy media. Voids have higher temperatures and occupy a larger volume than the cooler, denser clouds. The material between $10^{4.4}$ and $10^{6} \mathrm{~K}$ arises in the small spherical region that is evacuated prior to the $\mathrm{SN}$ injection onto the grid. There the local density is reduced to 0.01 of its original values. The fractional volume this occupies remains small compared to the entire region inside the scale radius. See text for details.

out the clumps on timescales of

$$
\tau_{\text {cross }} \sim r_{\mathrm{s}} / c_{\mathrm{s}} \sim 100 \mathrm{pc} / 1 \mathrm{~km} \mathrm{~s}^{-1} \sim 100 \mathrm{Myr}
$$

where $r_{\mathrm{s}}$ is the scale radius of the Einasto halo and $c_{\mathrm{s}}$ is the thermal sound speed for a gas temperature of $\sim 10^{3} \mathrm{~K}$. This remains a significantly longer timescale than the $\mathrm{SN}$ evolution timescale, so we are justified in assuming equilibrium during the main evolution models, and a differential experiment where we can compare directly fractal and smooth models that have the same mean properties averaged over the distribution is valid.

Energy injection. To model the energy injection from $\mathrm{SNe}$, we insert a bubble of hot gas with the equivalent energy of $10^{51} \mathrm{erg}$, on the assumption that the SN energy has been thermalized on a scale much below our parsec-scale resolution. This high-pressure bubble expands and converts internal thermal energy to kinetic energy and radiative losses during the expansion.

For the smooth models with cooling, it is important to provide additional smoothing to the boundary of the injection region to avoid the grid cell errors that seed the well-known "carbuncle instability" (see Sutherland 2010). This is especially strong in the case of smooth radiative models because of the formation of radiative thin shells that are particularly susceptible to this. We reduce the impact of these effects by using an injection region with a 3 cell radius smoothed with a Gaussian kernel ( 2 cell FWHM).

We locate our injection region either at the center of the potential or off-centered at the half gas-mass radius $\left(r_{0.5 \mathrm{~g}}\right)$ listed in Table 3, found from integrating the mean density curves in Figure 4. In Figure 10, the injection region is shown for the M60 off-centered clumpy model.
We follow the evolution of the $\mathrm{SN}$ for $T_{0}=25 \mathrm{Myr}$, taking snapshots at $25 \mathrm{kyr}$ intervals. This timescale is now well established in starburst and Carina-like dwarf galaxies (Tolstoy et al. 2009; Sharp \& Bland-Hawthorn 2010). We analyze our results by summing the dynamically cold and total mass, and internal kinetic energy, within one scale radius of the central potential at each time step.

\section{RESULTS}

\subsection{Preionization Phase}

Our goal is to establish the low-mass limit of a spherical dark matter halo that can retain baryons after a single $\mathrm{SN}$ event. But first we consider the impact of the progenitor star on the surrounding gas prior to the $\mathrm{SN}$ explosion. The mechanical wind energy is completely dwarfed by the star's radiative phase for any progenitor of an SN.

We consider how the UV propagates in each of the four halos for both clumpy and smooth media that undergo cooling. This is a complex process, and so we include access to $3 \mathrm{D}$ movies that reveal how the ionization front develops. These can be found at: http://miocene.anu.edu.au/smallgalaxy. We show a time sequence of the full preionization phase and the subsequent SN explosive phase in two ways: (i) fixed reference frame; (ii) rotating reference frame. There are two other aids to visualization. First, we include half-tone figures that show critical time steps for the different models: M60 (Figures 12 and 13), M65 (Figures 14 and 15), and M70 (Figures 16 and 17). Second, we include color composite images in Figure 18 that directly compare the information embodied in Figures 1217 , i.e., the neutral and ionized components as the nebula develops before and after the SN event.

A summary of gas mass and energy retention after the preionization phase is given in Tables 5 and 6 , respectively. The initial gas masses inside two cardinal radii, $r_{\mathrm{s}}$ and $r_{\mathrm{vir}}$, are included for each model. The M60, M65, and M70 models retain essentially all of the gas within $r_{\text {vir }}$ for the cooling models, while the M55 case (not shown) is evacuated completely. For the adiabatic cases (not shown), the M60 halo is evacuated completely and the M65 halo appears to lose more than half the gas after $30 \mathrm{Myr}$. In this instance, as the gas reacts to the radiation field, it becomes photoheated, expands, smooths out, and drops in density as a result. The number of recombinations locally declines, and the radiation propagates into more of the surrounding gas.

In Figures 12-17, we observe a number of important facts about the preionization phase. For the centered models, the Strömgren radius of the $\mathrm{H}$ II region falls outside (M60), onto (M65), and inside (M70) the scale radius. From the zoomed figures, we observe how the photoheating smooths out the substructure. The effect is so strong that we observe a cellular structure as the ionization fronts of many expanding knots overlap, which is particularly clear in the color composite image (Figure 18). In the same figure, we see that broad channels appear where the radiation is able to escape to the outer halo over a substantial solid angle. Even before the SN event, the expanding Strömgren sphere has produced a diffuse neutral shell of gas around the hot bubble cavity. In the offcentered models, the expanding Strömgren sphere compresses the core region gas, and the radiation is blocked from propagating over at least $2 \pi$ sr. This becomes important in 

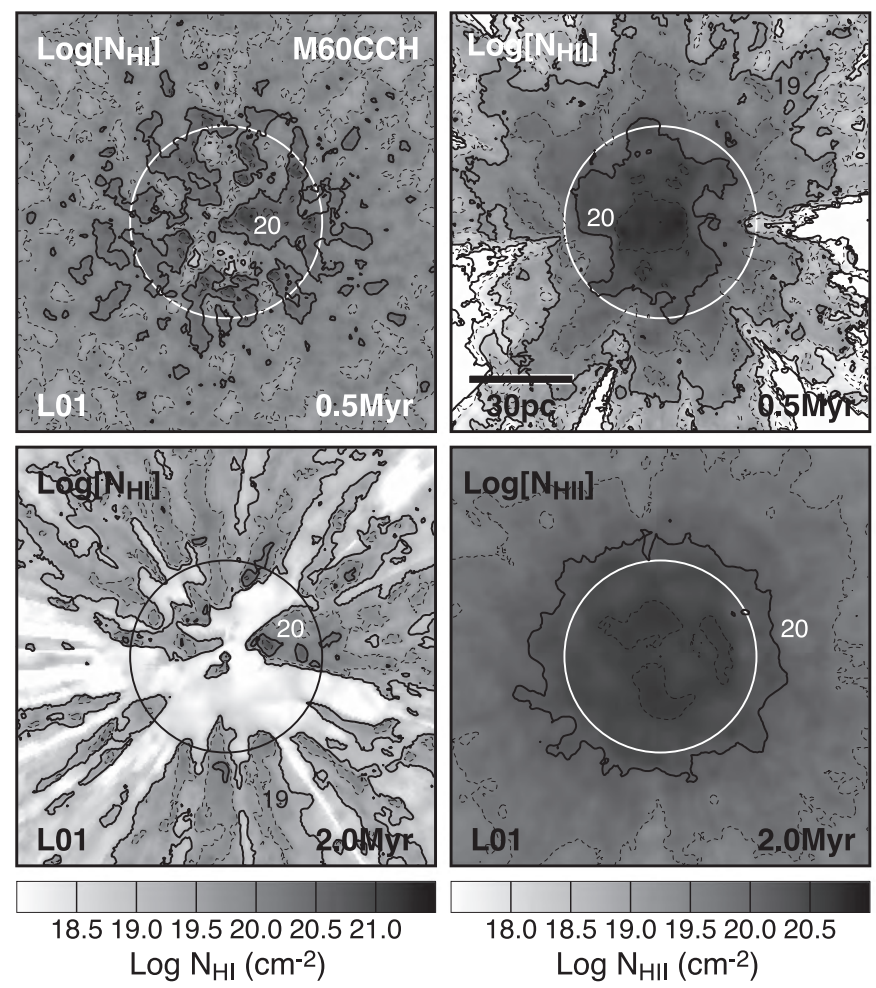

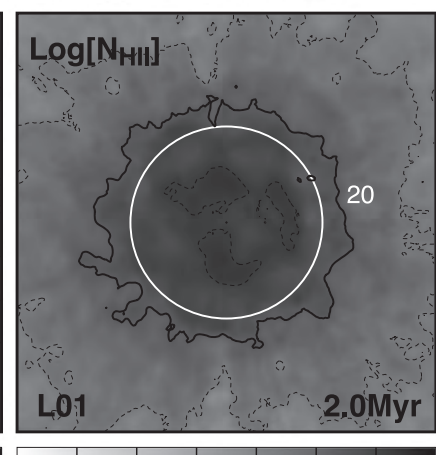

18.018 .519 .019 .520 .020 .5 $\log N_{H I I}\left(\mathrm{~cm}^{-2}\right)$
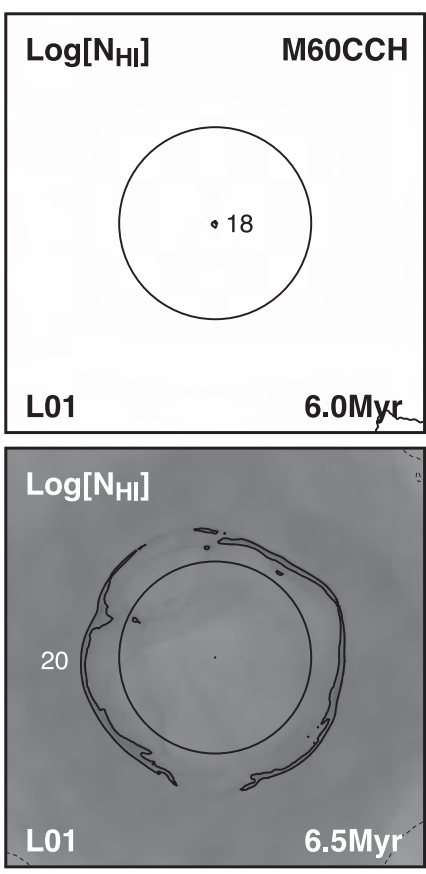

18.519 .019 .520 .020 .521 .0
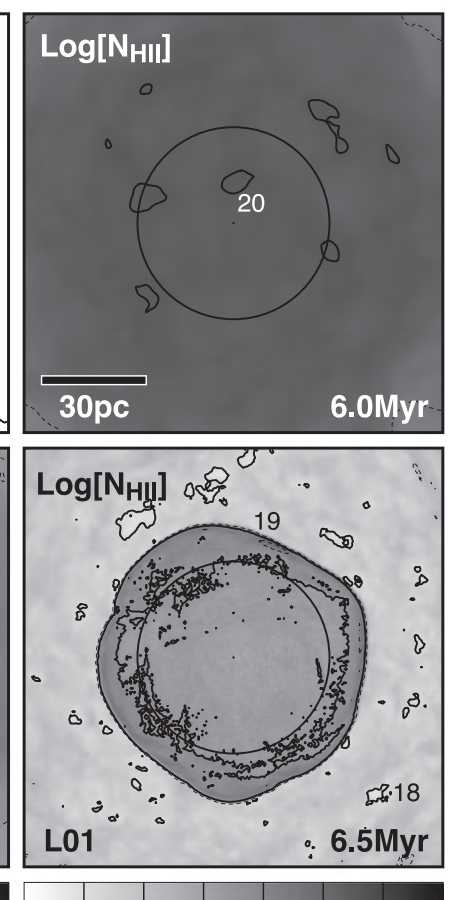

18.018 .519 .019 .520 .020 .5 $\log \mathrm{N}_{\mathrm{HII}}\left(\mathrm{cm}^{-2}\right)$

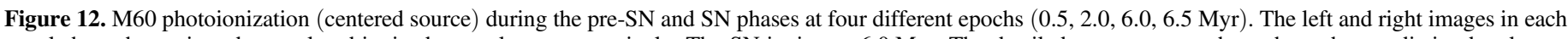

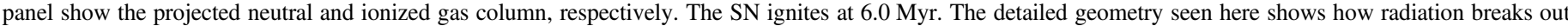

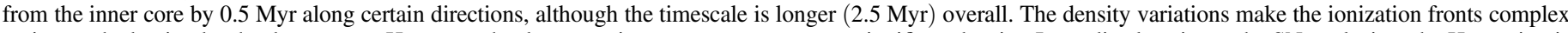

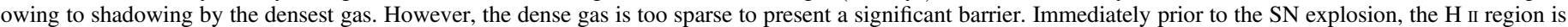

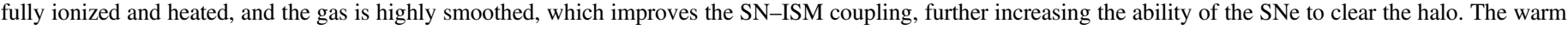
gas is less bound than initially as well, and by 6.5 Myr the SN remnant has cleared the core region completely.
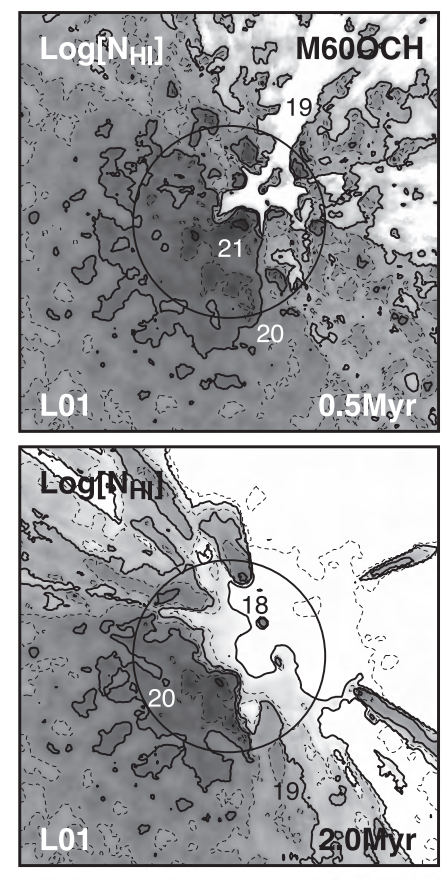

18.519 .019 .520 .020 .521 .0 $\log \mathrm{N}_{\mathrm{HI}}\left(\mathrm{cm}^{-2}\right)$
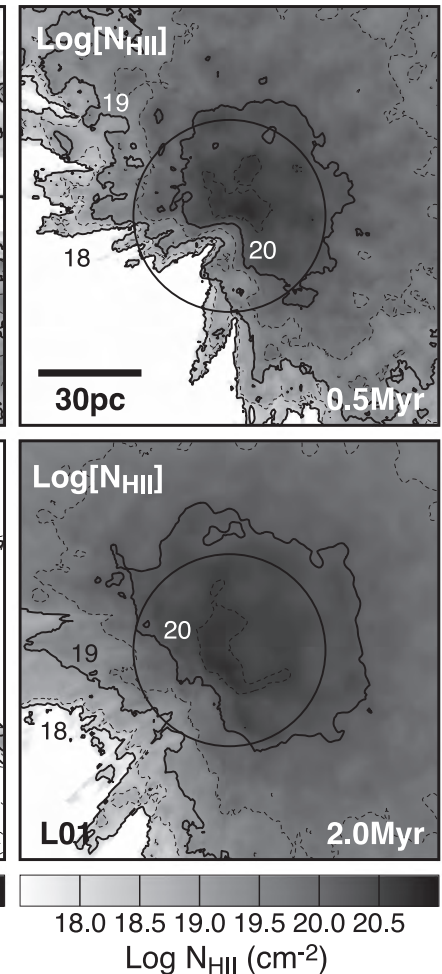
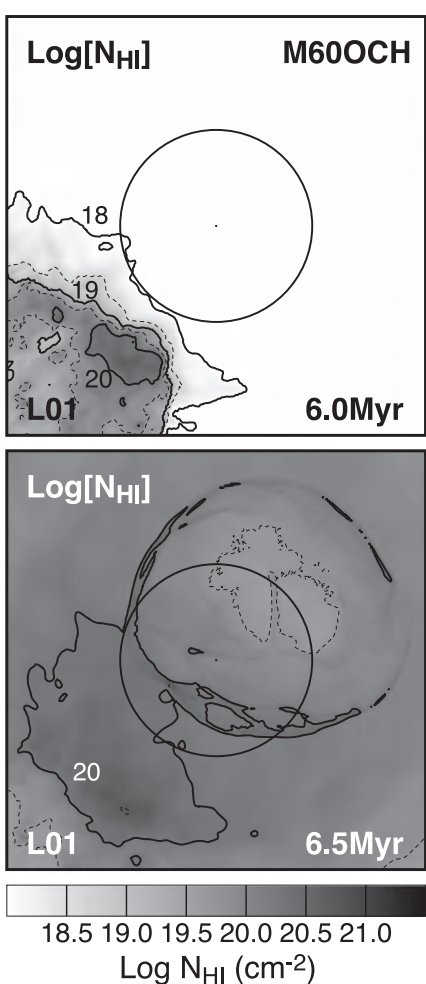
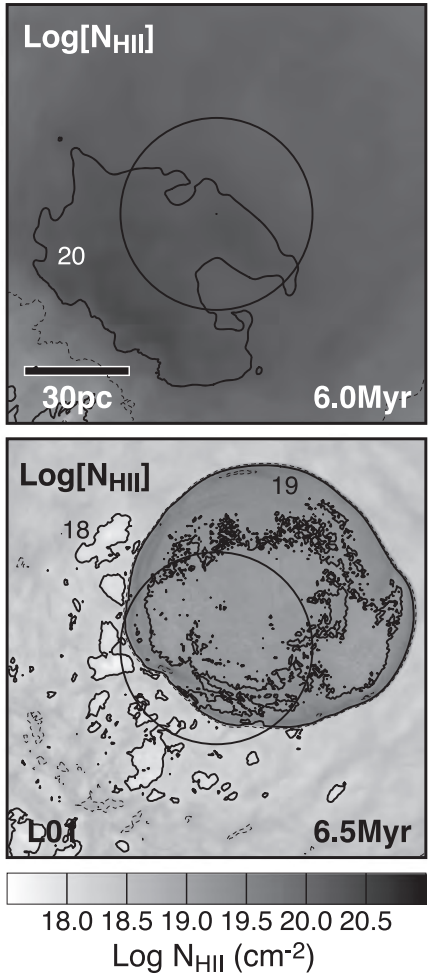

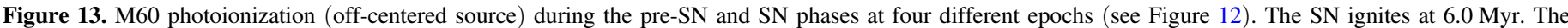

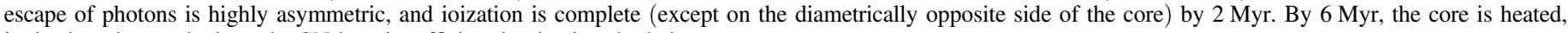
ionized, and smoothed, so the SN is quite efficient in clearing the halo. 

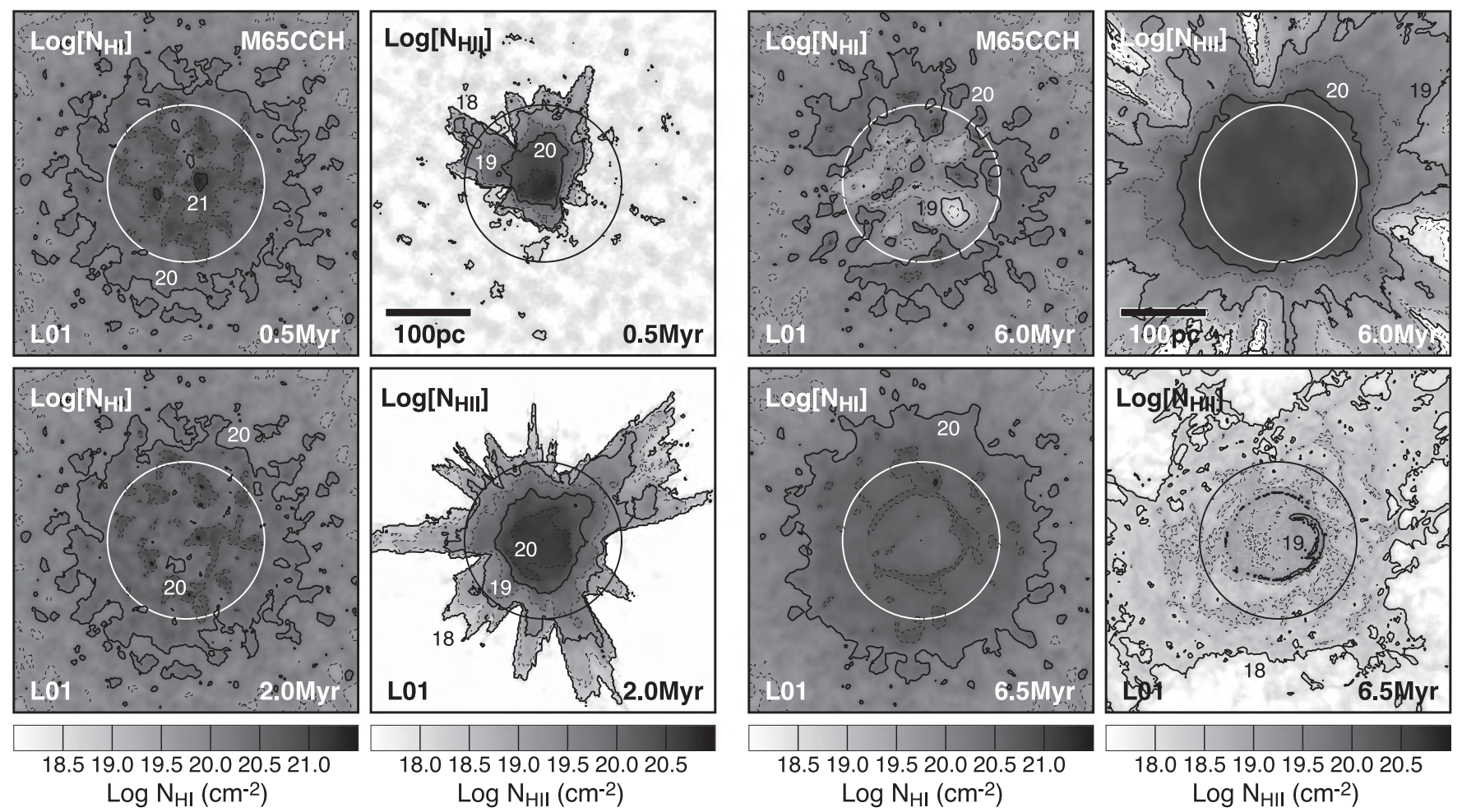

Figure 14. M65 photoionization (centered source) during the pre-SN and SN phases at four different epochs (see Figure 12). The SN ignites at 6.0 Myr. The nonuniform gas presents low-density channels, so that by $2.0 \mathrm{Myr}$, the spiky ionization fronts have escaped the core in the central model. By $6.0 \mathrm{Myr}$, the entire core region is photoionized.
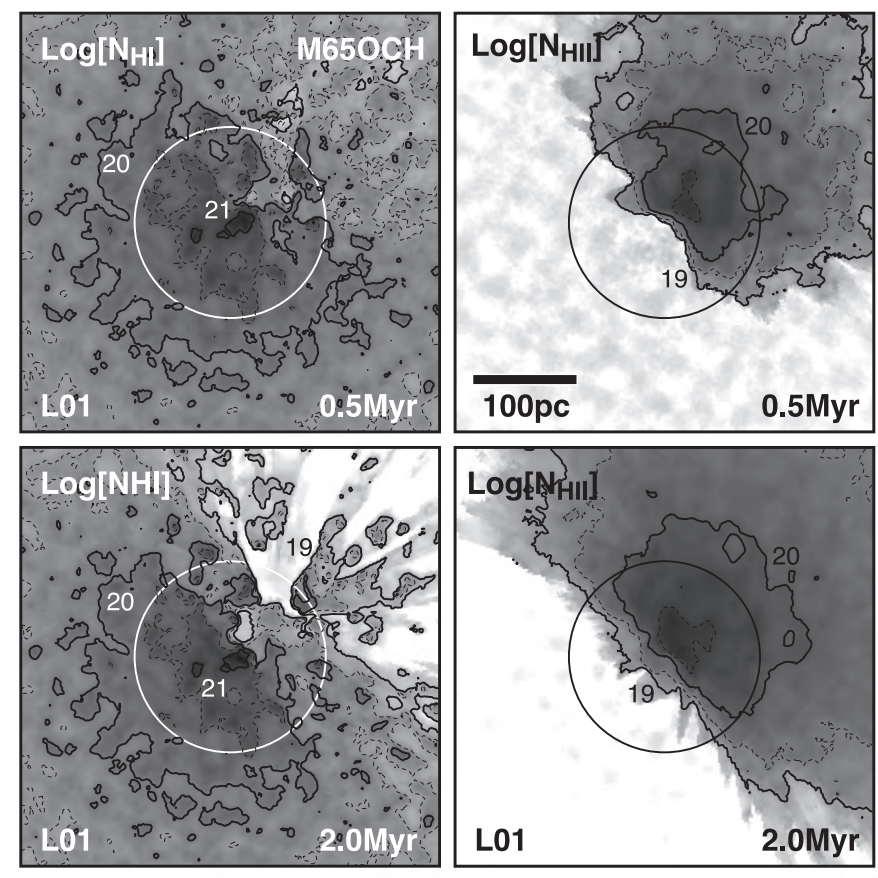

18.519 .019 .520 .020 .521 .0 $\log \mathrm{N}_{\mathrm{HI}}\left(\mathrm{cm}^{-2}\right)$

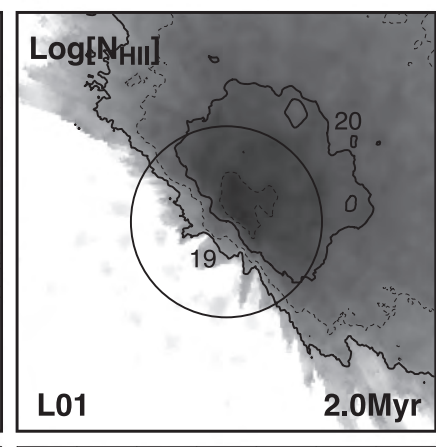

18.018 .519 .019 .520 .020 .5 $\log \mathrm{N}_{\mathrm{HII}}\left(\mathrm{cm}^{-2}\right)$
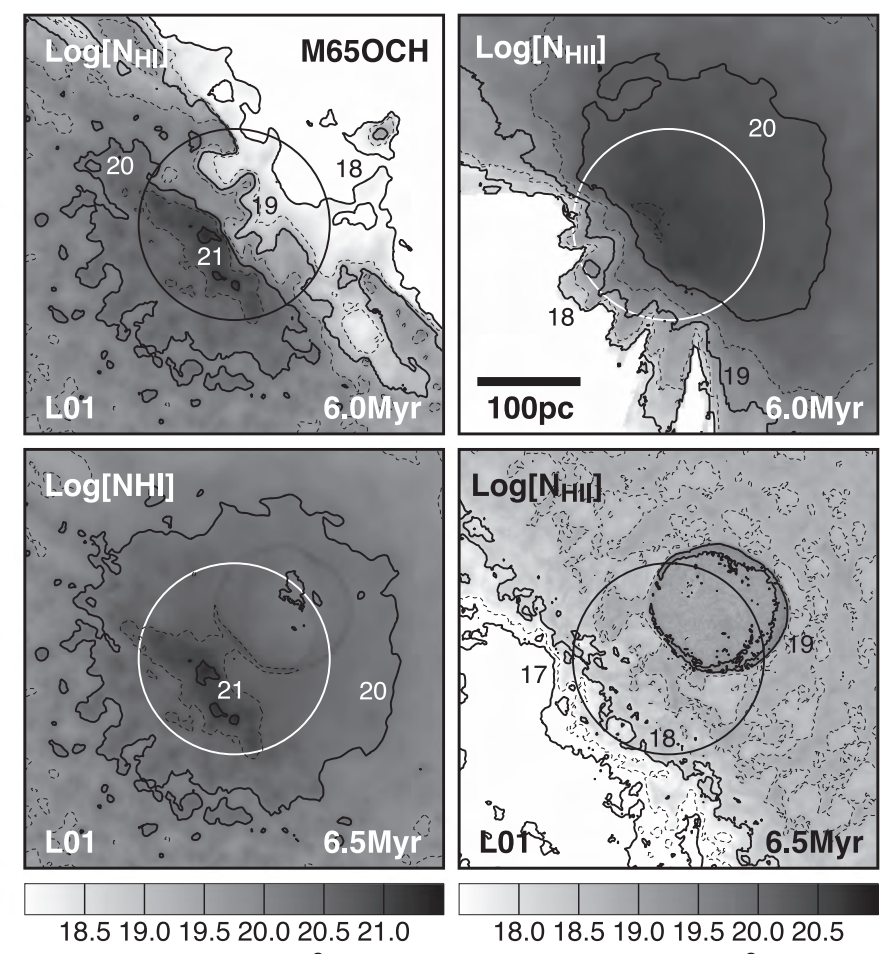
$\log \mathrm{N}_{\mathrm{HI}}\left(\mathrm{cm}^{-2}\right)$

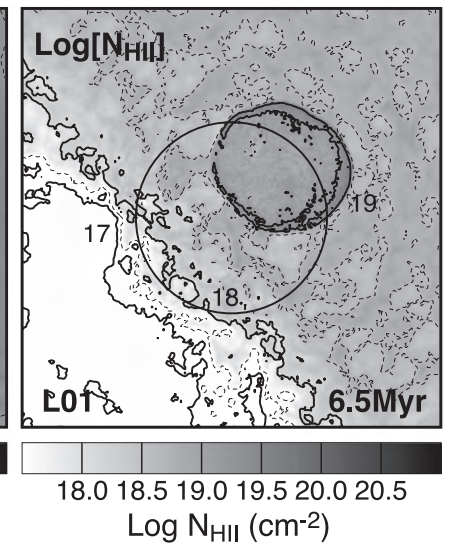

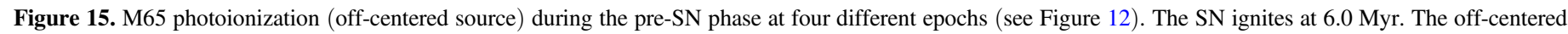

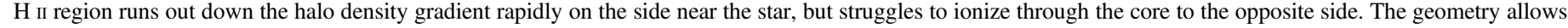

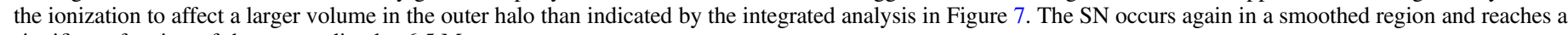
significant fraction of the core radius by $6.5 \mathrm{Myr}$. 

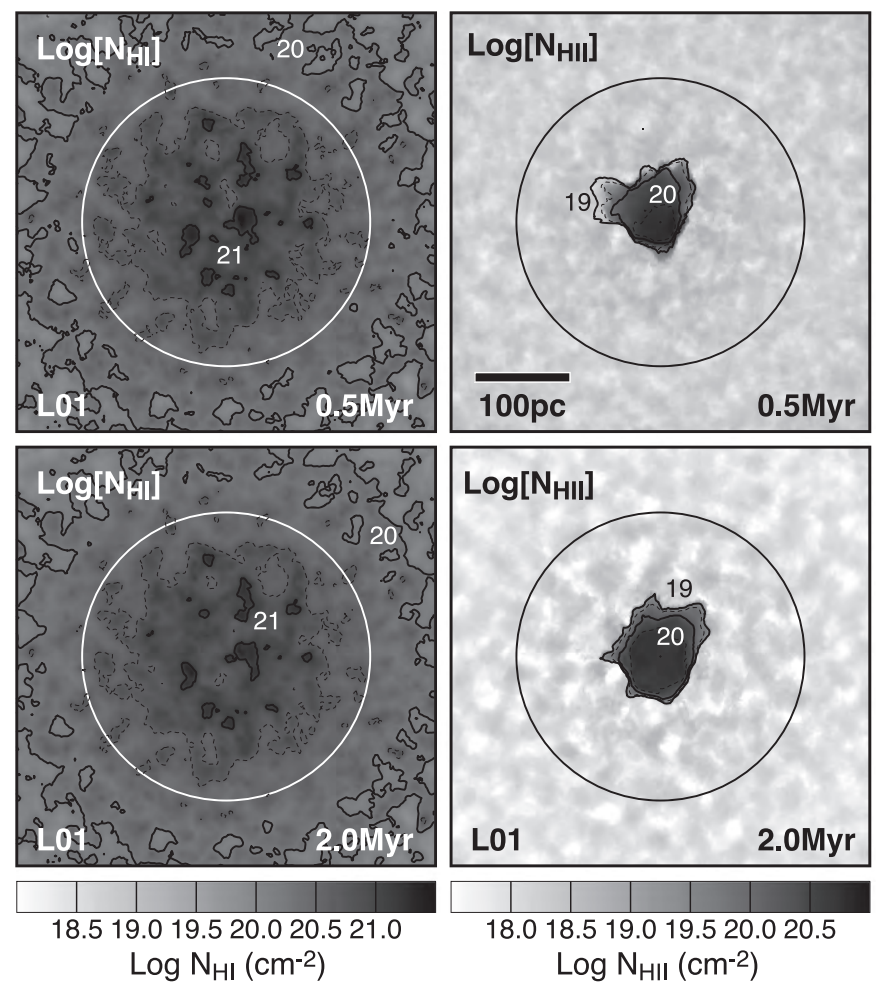

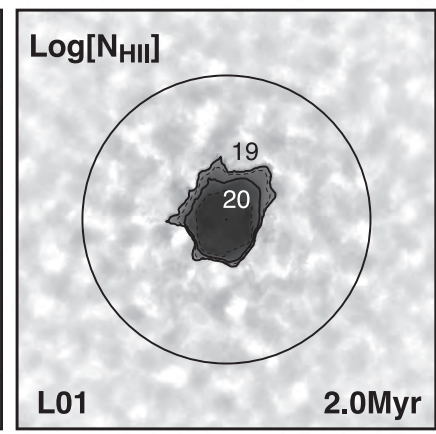

18.018 .519 .019 .520 .020 .5 $\log \mathrm{N}_{\mathrm{HII}}\left(\mathrm{cm}^{-2}\right)$
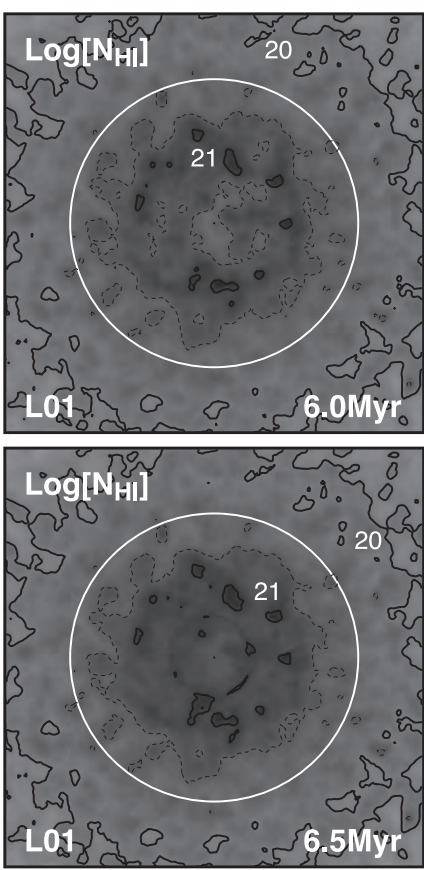

18.519 .019 .520 .020 .521 .0
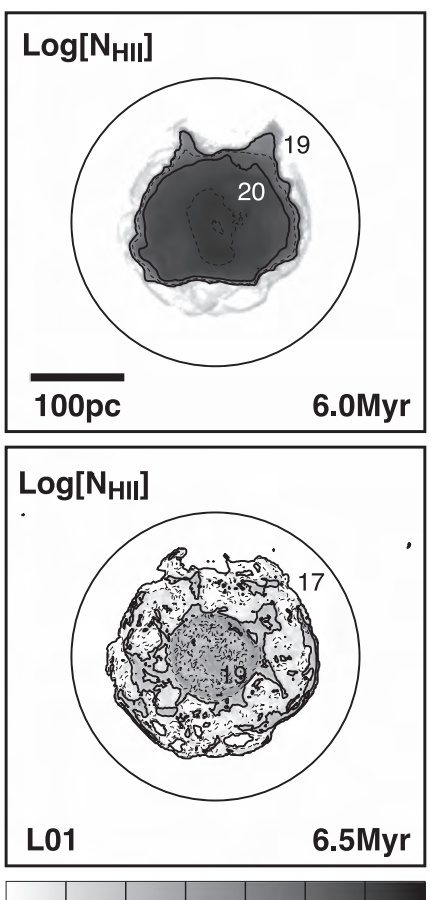

18.018 .519 .019 .520 .020 .5 $\log \mathrm{N}_{\mathrm{HII}}\left(\mathrm{cm}^{-2}\right)$

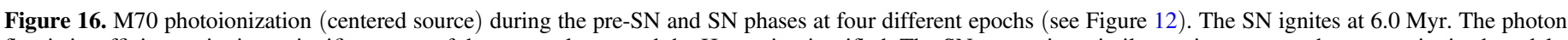

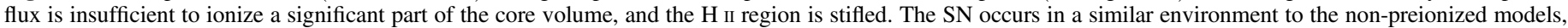
and the outcomes are similar. Essentially all of the gas survives the impact of the SN event.
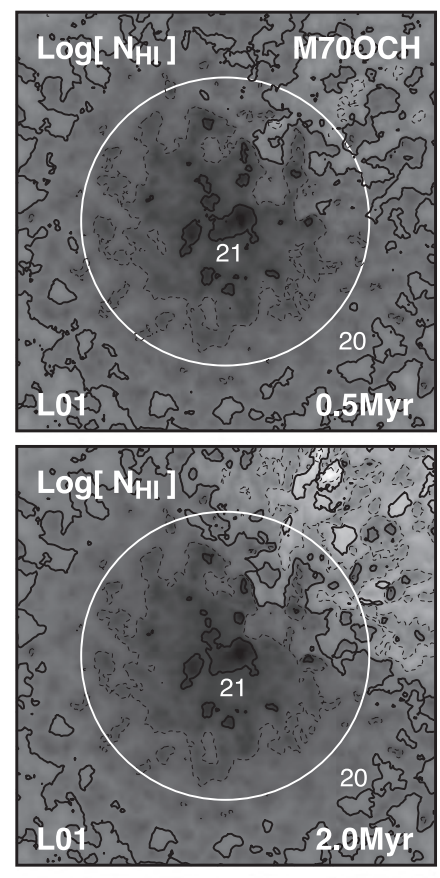

18.519 .019 .520 .020 .521 .0 $\log \mathrm{N}_{\mathrm{HI}}\left(\mathrm{cm}^{-2}\right)$
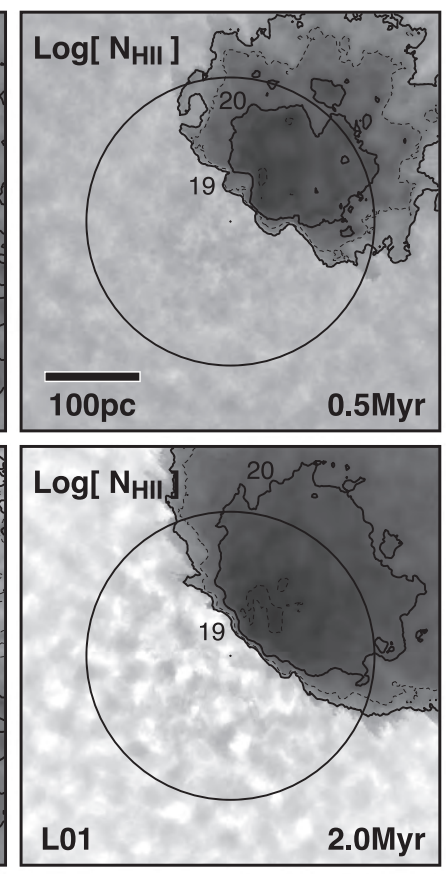

18.018 .519 .019 .520 .020 .5 $\log N_{H I I}\left(\mathrm{~cm}^{-2}\right)$
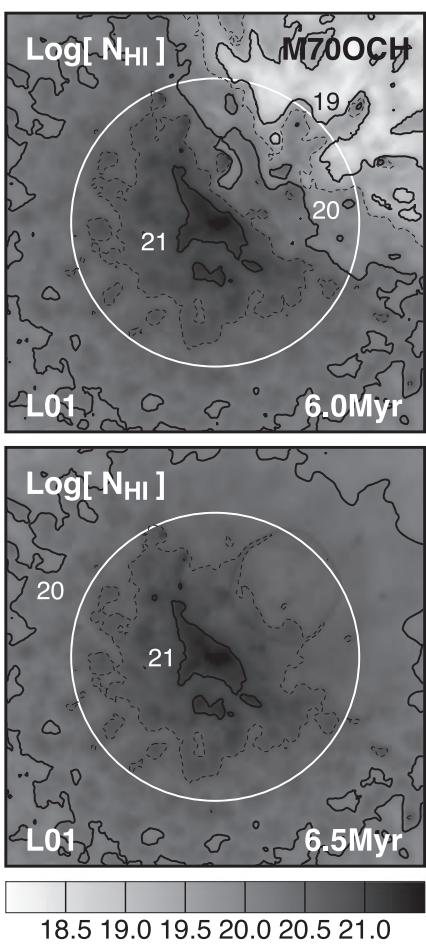
$\log \mathrm{N}_{\mathrm{HI}}\left(\mathrm{cm}^{-2}\right)$
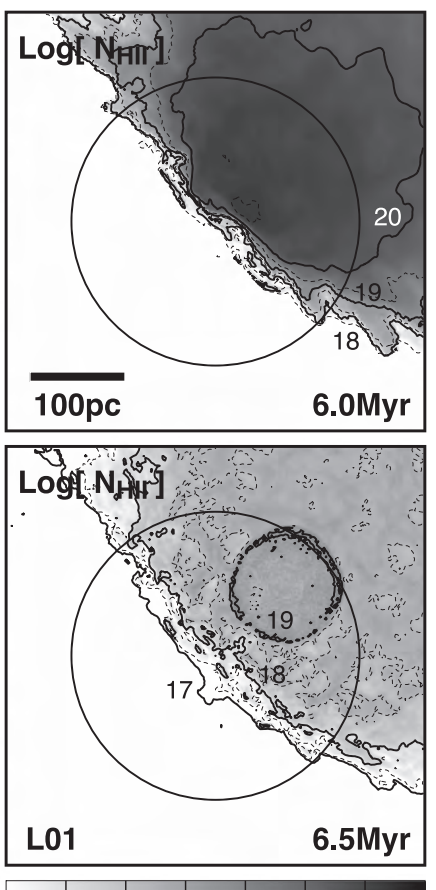

18.018 .519 .019 .520 .020 .5 $\log \mathrm{N}_{\mathrm{HII}}\left(\mathrm{cm}^{-2}\right)$

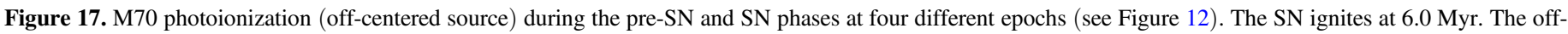

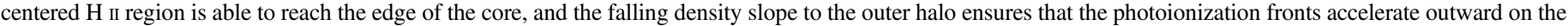

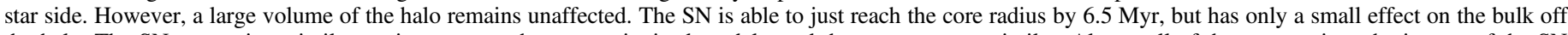

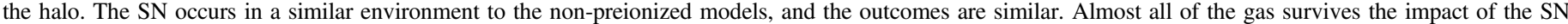
event. 

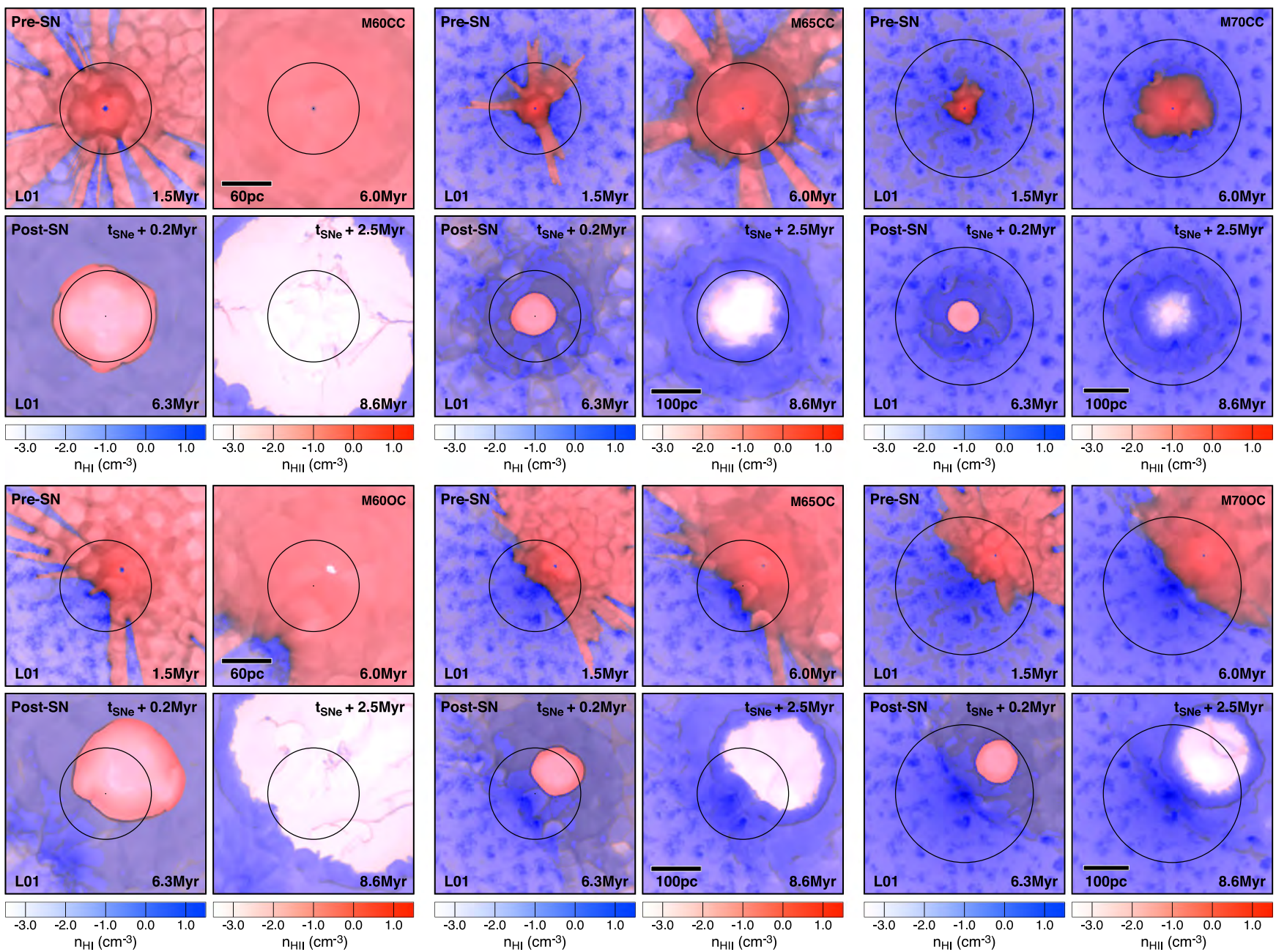

Figure 18. Development of the preionization and SN phases for the M60, M65, and M70 cases for four different epochs; the top three panels are for a central source, and the lower three panels are the corresponding off-centered cases. The snapshots show the distribution of ionized and neutral gas before, during, and after the SN event at 6.0 Myr. This figure is a color composite of the information contained in Figures 12-17. Each image is a density slice through the center of the halo. The red shows the distribution of fully ionized gas; the blue is the neutral gas; mauve corresponds to partially ionized gas.

Table 5

Percentage of Initial Mass Retained inside $r_{\mathrm{s}}$ and $r_{\mathrm{vir}}$ for Cooling Models

\begin{tabular}{|c|c|c|c|c|}
\hline \multirow{2}{*}{$\begin{array}{l}\text { Model } \\
\text { Time }^{\mathrm{a}}\end{array}$} & \multicolumn{2}{|c|}{ Inside $r_{s}$} & \multicolumn{2}{|c|}{ Inside $r_{\text {vir }}$} \\
\hline & Central & $\overline{\text { Off-Center }}$ & Central & $\overline{\text { Off-Center }}$ \\
\hline М60H & \multicolumn{2}{|c|}{$M_{\mathrm{g}, \mathrm{s}}: 1.91 \mathrm{E}+04 M_{\odot}$} & \multicolumn{2}{|c|}{$M_{\mathrm{g}, \text { vir }}: 1.22 \mathrm{E}+05 M_{\odot}$} \\
\hline 0 Myr & 12.7 & 16.1 & 97.6 & 97.1 \\
\hline 5 Myr & 0.03 & 0.06 & 89.3 & 88.4 \\
\hline 25 Myr & 0.01 & 0.21 & 0.98 & 6.10 \\
\hline М65H & \multicolumn{2}{|c|}{$M_{\mathrm{g}, \mathrm{s}}: 6.61 \mathrm{E}+04 M_{\odot}$} & \multicolumn{2}{|c|}{$M_{\mathrm{g}, \mathrm{vir}}: 3.74 \mathrm{E}+05 M_{\odot}$} \\
\hline 0 Myr & 50.3 & 78.8 & 100.0 & 99.4 \\
\hline $5 \mathrm{Myr}$ & 2.42 & 41.7 & 99.7 & 97.9 \\
\hline $25 \mathrm{Myr}$ & 13.8 & 33.9 & 81.5 & 73.3 \\
\hline М70H & \multicolumn{2}{|c|}{$M_{\mathrm{g}, \mathrm{s}}: 2.38 \mathrm{E}+05 M_{\odot}$} & \multicolumn{2}{|c|}{$M_{\mathrm{g}, \mathrm{vir}}: 1.16 \mathrm{E}+06 M_{\odot}$} \\
\hline 0 Myr & $102.6^{\mathrm{b}}$ & 98.2 & 100.0 & 100.0 \\
\hline $5 \mathrm{Myr}$ & $106.2^{\mathrm{b}}$ & 92.8 & $100.2^{\mathrm{b}}$ & $100.1^{\mathrm{b}}$ \\
\hline 25 Myr & $121.5^{\mathrm{b}}$ & $107.0^{\mathrm{b}}$ & $102.6^{\mathrm{b}}$ & $101.2^{\mathrm{b}}$ \\
\hline
\end{tabular}

Notes. Bold: $<50 \%$ retained. The time at $0 \mathrm{Myr}$ is when the SN event takes place, at which point the preionization phase ends. Entries in bold fall below $50 \%$.

${ }^{\mathrm{a}} 0 \mathrm{Myr}=t_{\mathrm{SNe}},=6.1 \mathrm{Myr}$ from start of simulation.

${ }^{\mathrm{b}}$ Increased mass after some infall. the post-SN phase because substantial gas begins to re-accrete to the center along this axis.

\subsection{SN Phase}

Our complete set of simulations (see Table 3) comprises 24 cases for SN events without preionization with the massive progenitor, and 6 cases where the ionization of the SN progenitor is included. All 30 cases were run at L00 and L01, with a subset of the cooling models run at L02 to allow adequate sampling of the developing SN shell (see Figure 5). L02 is always found to be adequate to resolve the metalenriched shell, which starts as a diffuse hot envelope that becomes thinner under expansion. The models do not suffer the overcooling through inadequate resolution identified by Dalla Vecchia \& Schaye (2012).

We discuss in detail only the clumpy, cooling models for the centered and off-centered SN cases where the progenitor ionization is included. We present these results for three different halo masses (M60, M65, M70). ${ }^{7}$ Tables 5 and 6 include the total gas mass and energy retained within $r=r_{\mathrm{s}}$ and $r=r_{\mathrm{vir}}$ after periods of $t=5 \mathrm{Myr}$ and $t=25 \mathrm{Myr}$. The halos

All simulations can be viewed at http://miocene.anu.edu.au/smallgalaxy. 
Table 6

Percentage of Initial Energy Retained inside $r_{\mathrm{s}}$ and $r_{\mathrm{vir}}$ for Cooling Models. The time at $0 \mathrm{Myr}$ is when the $\mathrm{SN}$ event takes place, at which point the preionization phase ends. Entries in bold fall below $50 \%$.

\begin{tabular}{|c|c|c|c|c|}
\hline \multirow{2}{*}{$\begin{array}{l}\text { Model } \\
\quad \text { Time }^{\mathrm{a}}\end{array}$} & \multicolumn{2}{|c|}{ Inside $r_{\mathrm{s}}$} & \multicolumn{2}{|c|}{ Inside $r_{\text {vir }}$} \\
\hline & Central & Off-center & Central & Off-center \\
\hline М60Н & \multicolumn{2}{|c|}{$E_{s}: 4.52 \mathrm{E}+48 \mathrm{erg}$} & \multicolumn{2}{|c|}{$E_{\text {vir }}: 2.75 \mathrm{E}+49 \mathrm{erg}$} \\
\hline Pre-SNe & 237.7 & 299.1 & 1725 & 1647 \\
\hline Post-SNe & $1.74 \mathrm{E}+04$ & $1.79 \mathrm{E}+04$ & 4365 & 4374 \\
\hline 5 Myr & 1.90 & 1.00 & 1242 & 1179 \\
\hline $25 \mathrm{Myr}$ & 0.06 & 0.31 & 1.93 & 9.32 \\
\hline М65H & \multicolumn{2}{|c|}{$E_{s}: 3.19 \mathrm{E}+49 \mathrm{erg}$} & \multicolumn{2}{|c|}{$E_{\mathrm{vir}}: 1.73 \mathrm{E}+50 \mathrm{erg}$} \\
\hline Pre-SNe & 327.4 & 318.1 & 325.3 & 402.4 \\
\hline Post-SNe & 2613 & 2596 & 722.1 & 797.3 \\
\hline 5 Myr & 2.71 & 50.7 & 232.7 & 255.4 \\
\hline 25 Myr & $20.1^{b}$ & 29.6 & 101.4 & 91.5 \\
\hline М70H & \multicolumn{2}{|c|}{$E_{s}: 3.45 \mathrm{E}+50$ erg } & \multicolumn{2}{|c|}{$E_{\mathrm{vir}}: 1.63 \mathrm{E}+51 \mathrm{erg}$} \\
\hline Pre-SNe & 92.2 & 89.8 & 59.4 & 81.8 \\
\hline Post-SNe & 328.5 & 319.2 & 107.2 & 126.6 \\
\hline 5 Myr & 64.6 & 57.6 & 54.1 & 64.3 \\
\hline 25 Myr & $84.9^{b}$ & $67.8^{\mathrm{b}}$ & $61.8^{\mathrm{b}}$ & 62.4 \\
\hline
\end{tabular}

Notes. Bold: $<50 \%$ retained. Pre-SNe: 50 kyr prior to SNe. Post-SNe: $10 \mathrm{kyr}$ after SNe.

${ }^{\mathrm{a}} 0 \mathrm{Myr}=t_{\mathrm{SNe}},=6.1 \mathrm{Myr}$ from start of simulation.

${ }^{\mathrm{b}}$ Increased mass after some infall.

that retain less than $50 \%$ of their original mass or energy have their retention fractions shown in boldface.

Each of the models (M60, M65, M70) is discussed in more detail below. In Figures 12-17, we present time-dependent 2D slices of the gas distribution before and after the SN explosion. These are not summed along the third dimension and therefore do not illustrate the full extent of the ionized gas (see Figure 18). Unless otherwise stated, all computations are performed over a $648^{3}$ grid with two levels of the hierarchy (L00, L01) illustrated in Figure 5 (see Section 3.4). In a subset of cases, it was necessary to investigate a three-level hierarchy (L00, L01, L02) over a $1944^{3}$ grid, which we clarify below.

It is worthwhile to pay particular attention to Figure 19. Here we present $1 \mathrm{D}$ plots of the evolution of the gas mass $(\mathrm{A}-\mathrm{F})$ and energy $(\mathrm{G}-\mathrm{L})$ retention fraction over much longer timescales than shown in the half-tone figures (up to $25 \mathrm{Myr}$ ). In each plot, we present two sets of curves for retention within $r \leqslant r_{\text {vir }}$ (upper) and $r \leqslant r_{\mathrm{s}}$ (lower).

The left panels (A, C, E, G, I, K) show all combinationsclumpy (solid curves) versus smooth (dashed curves), offcentered (thin curves) versus centered (thick curves), adiabatic (red curves) versus cooling (black curves). These plots are particularly illustrative and allow us to discuss the role of internal and kinetic energy in the developing explosion. For all models, the adiabatic cases (red curves) confirm earlier work in that there is little or no gas retention (Mac Low \& Ferrara 1999). In the right panels (B, D, F, H, J, L), we show only the cooling clumpy models for the centered and offcentered cases; the gas retention is clearly more favorable for the larger halo masses.

One important insight emerges that explains why we have arranged the figures in this way. In each row, we compare two distinct halo masses (e.g., M55 versus M60 in the top row) to illustrate the impact of preionization due to the $\mathrm{SN}$ progenitor.
The left panels (e.g., A) have no preionization, in contrast to the right panels (e.g., B), which include this effect. Broadly speaking, the results on retention are similar. The same holds true when we compare M60 models without preionization to M65 models with preionization, and M65 models without preionization to M70 models with preionization. These are important limiting cases when one considers enrichment in halos that do not have an important preionization phase, e.g., for star formation histories limited to low mass and/or SNe I.

\subsection{M60 Models}

The time-dependent behaviors of the M60 central and offcentered explosions are illustrated in Figures 12 and 13, respectively. The evolutionary phases of an explosive wind are well understood analytically (Weaver et al. 1977), although we see additional substructure due to the complexity of the initial medium. The SN ignites within a fully ionized, diffuse nebula confined by a neutral gas shell. A dense radiative shell quickly forms and drives a shock into the neutral shell. The momentum-driven shell does not stall and essentially evacuates all gas within the halo after $15 \mathrm{Myr}$. In the off-centered case, one hemisphere is initially evacuated within $15 \mathrm{Myr}$, with the opposite hemisphere losing most but not all of its gas. At late times, there is some re-accretion from the far side, leading to $<10 \%$ gas retention within the core regions. The energy input from the preionization phase, in combination with the $\mathrm{SN}$ explosive energy, conspires to doom the M60 halos to invisibility.

Numerical artifacts. In order to examine the impact of artifacts, this case is treated with a three-level grid. No such anomalies are seen in the preionization phase. In the SN event, numerical artifacts evident in the first $600 \mathrm{kyr}$ near the origin (Figure 20) are caused by the carbuncle instability (see Sutherland 2010). This effect is amplified by strong cooling in the early stages $(<125 \mathrm{kyr})$ and leads to the "clover leaf" pattern at 6.3 Myr inside $r_{\mathrm{s}}$ (L01). Our algorithm stabilizes the instability when the radiative thin shell becomes only partially resolved ( $<3$ cells thick) at later times, such that any structure that evolves in the interaction between the spherical shell and the Cartesian grid remains at all later stages. The instability is strongest where the radiative shock front becomes orthogonal to the underlying grid, in this case along the Cartesian axes, giving rise to the boxy structures with apexes along the $x$ - and $y$-axes seen here at $16 \mathrm{Myr}$ (L00). After the local density has dropped so that the blast wave is no longer radiative, the structure then simply expands with the now adiabatic blast. The additional L2 level is needed to better resolve the early blast, and the remaining visible non-spherical structure has radius errors of approximately $10 \%-15 \%$.

Gas retention and energetics. We now discuss the issue of gas retention in the M60 models in the context of the energy (internal+kinetic) of the disturbed gas. Table 6 shows the amount of energy retained at critical radii, $r=r_{\mathrm{s}}$ and $r=r_{\mathrm{vir}}$, immediately before and after the $\mathrm{SN}$ explodes, and at 5 and $25 \mathrm{Myr}$ after the SN event. We show density slices through our clumpy M60 models over this time frame in Figures 20 and 21.

We recall that during the preionization phase, radiation leaks out from the center in the first few Myr, leading to a fully developed, low-density nebula by $6 \mathrm{Myr}$ (see Section 3.5). In Figure 20, the central SN explodes into a warm low-density nebula, superheats the gas through shock heating, and removes 

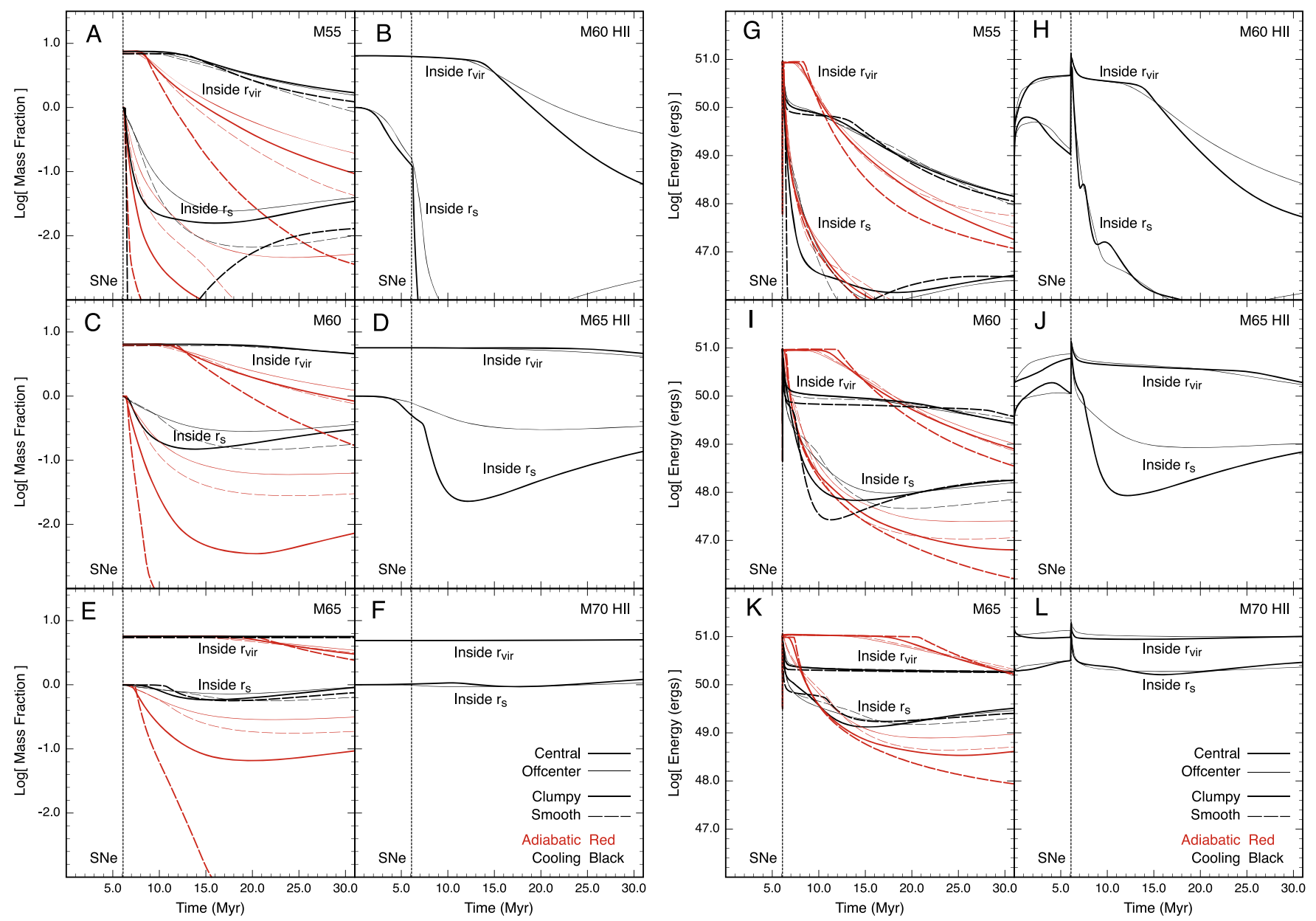

Figure 19. Gas mass (A-F) and energy (G-L) retention inside the M55, M60, M65, and M70 models. Panels A, C, E and G, I, K are for SN explosions without preionization (SN Ia); panels B, D, F and H, J, L are for SN explosions with preionization (SN II). For the former, we start the models at the explosion, so there are no data prior to the event. The time of the SN event is shown as a vertical dashed line; the impact of preionization (see Table 5) is clearly evident. The key to all curves is given in both figures. The top set of curves in each panel is for quantities within a radius $r_{\text {vir }}$; the bottom set is for quantities within $r_{\mathrm{s}}$. The mass fraction is defined relative to the original gas mass within the scale radii as indicated at $t=0$. We show both the adiabatic (red curves) and cooling models (black curves) for both clumpy and smooth media. Consistent with earlier work, the smooth adiabatic case is much lossier than the case for cooling halos. Note that the adiabatic case is energy conserving until gas is lost over a boundary. In the energy retention figures, note that M70's explosive energy has only a marginal impact on the energy retained from the progenitor phase.

essentially all of the gas from the dark matter halo out to $r_{\mathrm{vir}}$ after 25 Myr. This is borne out in Table 5.

For the off-centered case in Figure 21, the progenitor SN is placed at the half gas-mass radius to reflect the fact that most stars form off-center. During the preionization phase, radiation leaks out from the center in the first few Myr (see Section 3.5), and a conic "zone of avoidance" is set up diametrically opposite the progenitor. By $6 \mathrm{Myr}$, a low-density nebula extends across most of the core region. The $\mathrm{SN}$ explodes into an off-centered, warm low-density nebula, superheats the gas through shock heating, and removes much of the gas asymmetrically from the dark matter halo out to $r_{\mathrm{vir}}$ after $25 \mathrm{Myr}$. The early zone of avoidance leads to far-side gas reaccreting into the core region after the $\mathrm{SN}$ event has passed, but less than $10 \%$ of the original gas remains within $r_{\text {vir }}$ on long timescales.

\subsection{M65 Models}

We now discuss gas retention in the M65 models within the context of the mass and energy of the disturbed gas. The timedependent behaviors of the M65 central and off-centered explosions are illustrated in Figures 14 and 15, respectively, before and including the explosion, and in Figures 22 and 23, respectively, after the SN event. To aid comparison, the projected neutral and ionized gases in Figures 14 and 15 are combined into a color composite in Figure 18.

Tables 5 and 6 show the percentage of mass and energy retained at $r_{\mathrm{s}}$ and $r_{\text {vir }}$ just before and after the $\mathrm{SN}$ explosion, and after delays of 5 and $25 \mathrm{Myr}$. We see that more gas mass is retained in the core in the off-centered case compared to the central case after $25 \mathrm{Myr}$ post-SN. The tabulated energy retentions are percentages relative to the binding energy of the gas bound by the dark halo. A system loses energy through either cooling (e.g., radiative, adiabatic) or mass loss; conversely, a system gains energy through either heating (e.g., shocks, UV radiation) or accretion. In the off-centered model, we see the effects of mass loss through the virial radius at the same time that the core is accreting gas.

In Figure 14, the SN ignites at $6.1 \mathrm{Myr}$ within a fully ionized, diffuse nebula confined by a neutral gas shell. A dense radiative shell quickly forms and drives a shock into the neutral shell. A lot of energy is radiated away in the time frame 0.2-2.5 Myr. The momentum-driven shell stalls at $1 \mathrm{Myr}$. At 


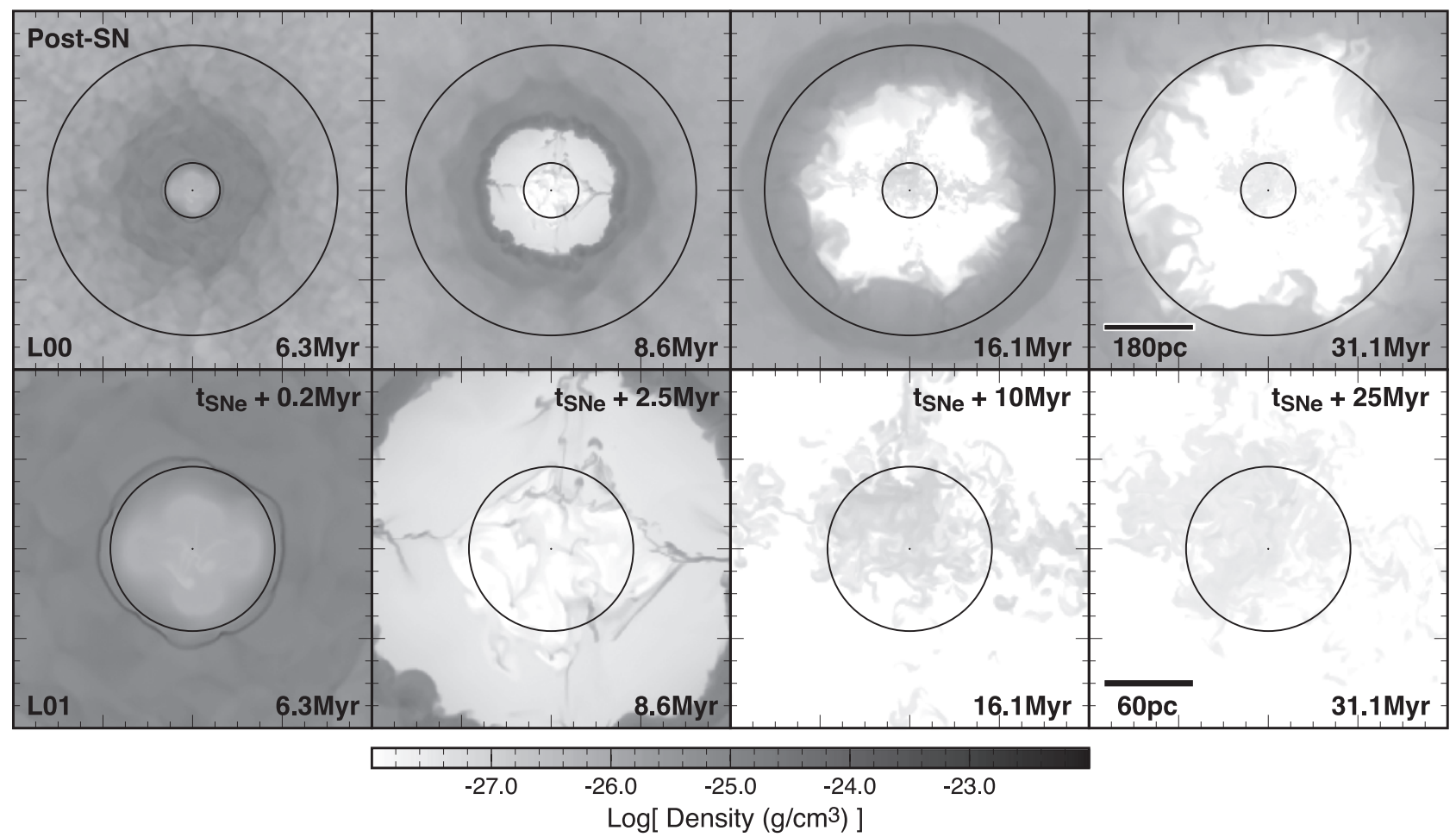

Figure 20. M60 central clumpy explosion commencing at the end of the progenitor ionization (preionization) phase. The SN explodes into a warm low-density nebula preheated by the progenitor. The SN wind superheats the gas through shock heating and removes essentially all of the gas from the dark matter halo out to $r_{\text {vir }}$ after $30 \mathrm{Myr}$. For the uppermost four panels (L00), the inner circle has radius $r_{\mathrm{s}}$, and the outer circle has radius $r_{\mathrm{vir}}$. Each panel has been magnified in the next four panels (L01); the circle has radius $r_{\mathrm{s}}$. All images are central plane density slices at $z=0.0$. Log of the normalized density variable in units of $1.0 \times 10^{-24} \mathrm{~g} \mathrm{~cm}{ }^{-3}$ : grayscale minimum $=-3.0$ (white), maximum $=2.0$ (black). A scale bar in parsecs is shown.

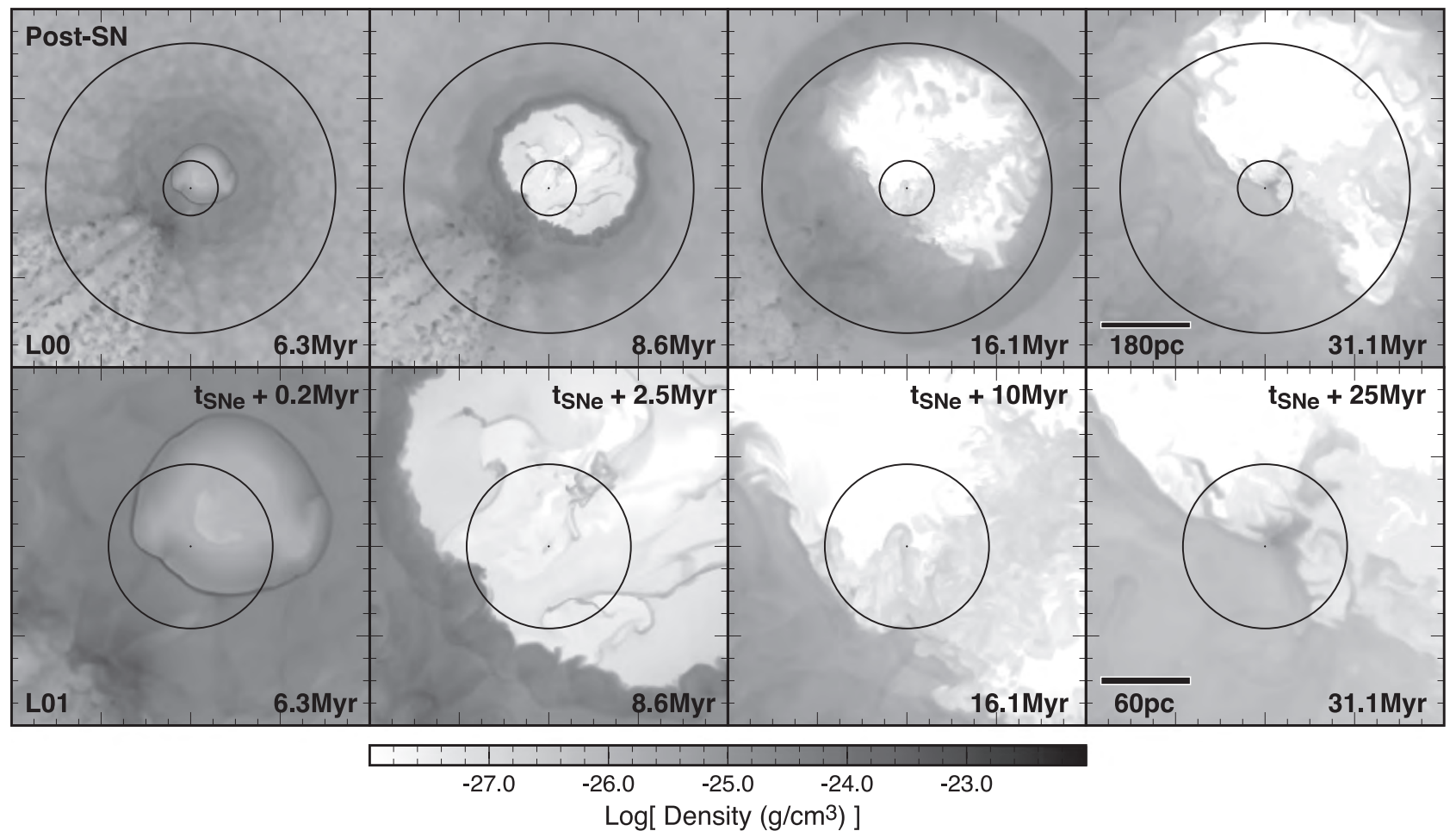

Figure 21. M60 off-centered clumpy explosion commencing at the end of the progenitor ionization phase; see Figure 20 for details. The star is placed at the half gasmass radius to reflect the fact that most stars form off-center. The conic "zone of avoidance" sets up in a region that is diametrically opposite the progenitor star; a lowdensity nebula extends across most of the core region before the SN ignites. The SN explodes into an off-centered, warm low-density nebula, superheats the gas through shock heating, and removes much of the gas asymmetrically from the dark matter halo out to $r_{\mathrm{vir}}$ after 30 Myr. The early zone of avoidance leads to far-side gas re-accreting into the core region after the $\mathrm{SN}$ event has passed, but less than $10 \%$ of the original gas remains within $r_{\mathrm{vir}}$ on long timescales. 


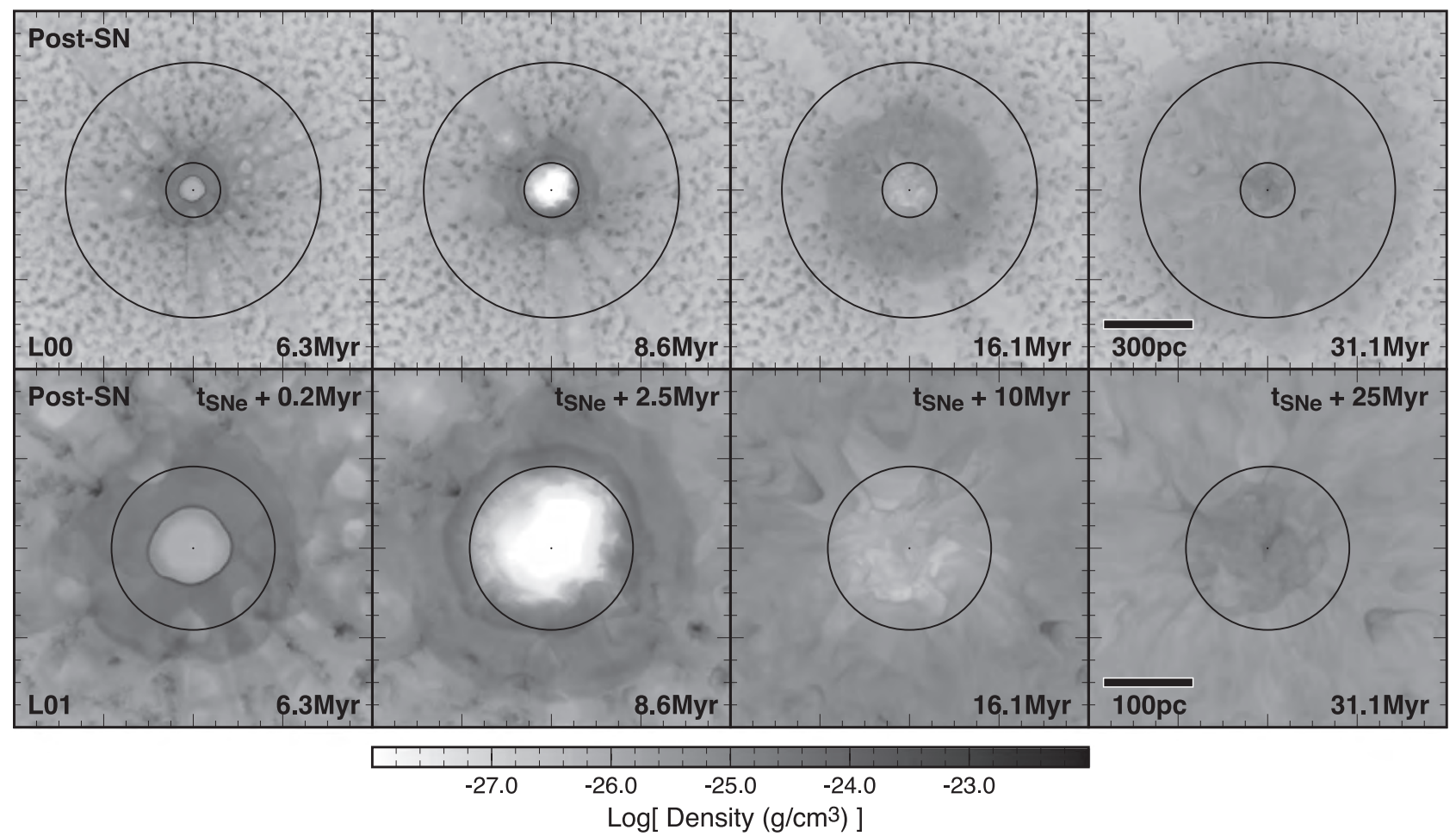

Figure 22. M65 central clumpy explosion commencing at the end of the progenitor ionization phase. For the uppermost four panels (L00), the inner circle has radius $r_{\mathrm{s}}$, and the outer circle has radius $r_{\mathrm{vir}}$. Each panel has been magnified in the next four panels (L01); the circle has radius $r_{\mathrm{s}}$. Unlike the M60 case, little radiation leaks out from the center for the first 6 Myr. The ionization is almost entirely restricted to the core region $\left(r<r_{\mathrm{s}}\right)$. The SN explodes into a warm, moderately dense nebula and superheats the gas through shock heating, but the gas cools as the nebula expands, which checks its progress. A diffuse ionized nebula forms that extends across the halo but is still bound by it. The core region cools and condenses, leading to re-accretion after the bubble collapses, starting as early as $3 \mathrm{Myr}$ after the SN explosion. All images are central plane density slices at $z=0.0$. Log of the normalized density variable in units of $1.0 \times 10^{-24} \mathrm{~g} \mathrm{~cm}^{-3}$ : grayscale minimum $=-3.0$ (white), maximum $=2.0$ (black). A scale bar in parsecs is shown.

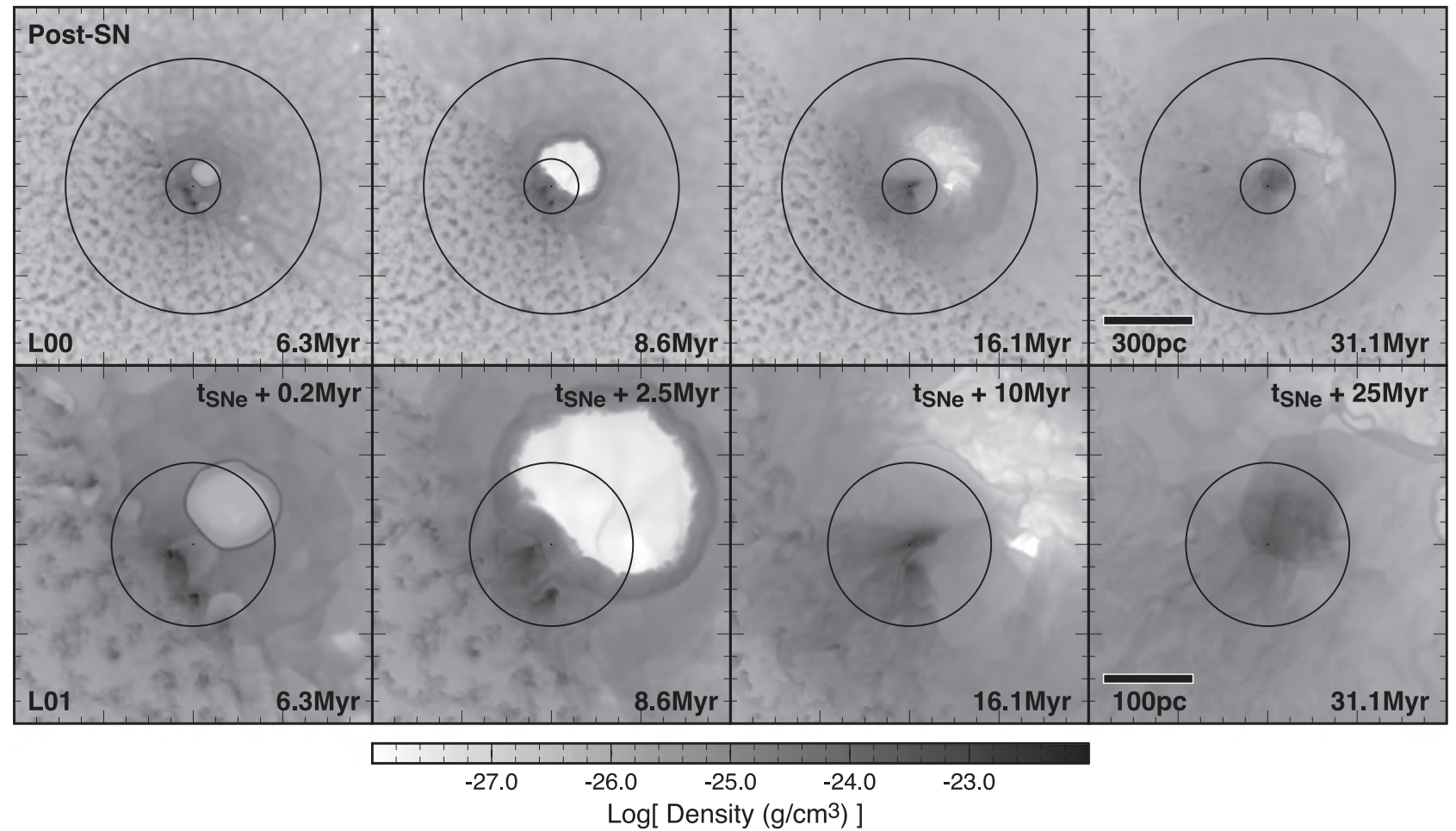

Figure 23. M65 Off-centered clumpy explosion commencing at the end of the progenitor ionization phase; see Figure 22 for details. The star is placed at the half gasmass radius to reflect the fact that most stars form off-center. The SN explodes into an off-centered, warm moderately dense nebula and superheats the gas through shock heating. This sends a blast wave into the surrounding nebula but fails to maintain the hot cavity that starts to collapse after 6 Myr. The early zone of avoidance leads to far-side gas re-accreting into the core region after the SN event has passed. On $30 \mathrm{Myr}$ timescales, there is essentially no gas loss from the dark halo. 
this point, the shock slows down and becomes non-radiative. The drop in temperature leads to a rapid increase in density. The neutral shell then becomes energy driven and more diffuse as it propagates outward at about the halo escape speed and ultimately stalls at the virial radius. Most of the gas just survives in the M65 progenitor ionization + SN model.

In Figure 15, the off-centered $\mathrm{H}$ II region runs out down the halo density gradient rapidly on the side near the star, but struggles to ionize through the core to the opposite side. The geometry allows the ionization to affect a larger volume in the outer halo than indicated by the integrated analysis in Figure 7. The SN occurs again in a smoothed region and reaches a significant fraction of the core radius by $6.5 \mathrm{Myr}$.

In these models, no more than half the gas survives the SN explosion. The surviving gas is enriched with the specific abundance yields of the discrete SN event. Our highestresolution simulations reveal why cooling is so effective in retaining gas compared to other factors. In the early stages, the super-hot metal-enriched SN ejecta exhibit strong cooling, leading to much of the explosive energy being lost. This remains true regardless of the assumed metallicity floor at the time of the explosion.

In Figures 22 and 23, we explore the post-SN phase over larger radial scales (LO0) and on longer timescales. The UV radiation from the SN progenitor escapes along some sight lines over the full spatial extent of the models. In both cases, the development of the SN-driven bubble is well defined. A thick $\mathrm{H}_{\mathrm{I}}$ shell forms by $10 \mathrm{Myr}$ ahead of the thin metalenriched shell, and this material starts to re-accrete to the core by $25 \mathrm{Myr}$. In the off-centered case, the central column densities are higher because the shock front wraps around the core and compresses it.

\subsection{M70 Models}

We now consider the retention of gas and energy in the M70 models. The $\mathrm{H}$ i and $\mathrm{H}$ II column densities before and just after the SN are shown in Figures 16 and 17, while Figures 24 and 25 show the Hi density evolution after the SN. For ease of comparsion, Figures 16 and 17 are also shown on the color composite image of Figure 18. The M65 models walk the tightrope between survival and disruption, retaining half their baryons at best. Tables 5 and 6 show percentages of gas and energy retained just before and after the explosion, as well as after 5 and 25 Myr. The M70 models clearly survive a single $\mathrm{SN}$ event, losing very little gas, and by $25 \mathrm{Myr}$ contain more gas than in their initial state as a result of accretion. As a result of cooling, the energies within both $r_{\mathrm{s}}$ and $r_{\mathrm{vir}}$ are much lower than the initial values at both 5 and $25 \mathrm{Myr}$, suggesting that the gas is not flowing outward.

For the M70 models, for the higher total gas mass, more than a single event could explode at a given epoch. But this is a complication that we do not consider here because the combined impact of the two events will depend on their masses (one is likely to be in the low-mass limit) and locations within the halo. In Figure 16, the central SN ignites at 6.0 Myr. The photon flux is insufficient to ionize a significant part of the core volume, and the $\mathrm{H}$ II region is stifled. In the off-centered case in Figure 17, the offcentered $\mathrm{H}_{\text {II }}$ region is able to reach the edge of the core, and the falling density slope to the outer halo ensures that the photoionization fronts accelerate outward on the side containing the star. The SN is able to just reach the scale radius by $6.5 \mathrm{Myr}$, but has only a small effect on the bulk of the halo.
The post-SN phase is shown in Figures 24 and 25. For the central SN model, the bubble at $t=2.5 \mathrm{Myr}$ is smaller than in the M65 case and sweeps up denser gas. By $10 \mathrm{Myr}$ the bubble collapses and dense gas accretes back to the center. In the off-centered case, the behavior is similar, although a small amount of radiation reaches the edge of the grid on the side nearest to the $\mathrm{SN}$, while the gas on the opposite side of the halo is largely unaffected. As in the M65 off-centered case, the central densities are increased at $2.5 \mathrm{Myr}$ when the shock compresses the core; however, the effect is weaker than in the M65 model because the shock is unable to wrap around the core.

Our models consider dwarf galaxies to be isolated, but in practice the vast majority reside in the extended halo of a host galaxy, albeit beyond the virial radius. In the M65 and M70 models, there is clear evidence of the expelled gas beginning to fall back after 20 Myr. A proper consideration of this gas requires that we consider the environment of the halo, which is beyond the scope of the current work. We leave this to a later study.

\subsection{Summary}

As described in the preceding sections, the summary plots for the single SN event models in Figure 19 (supported by Figures 12-17) reveal the following.

1. For all M55 models and M60 adiabatic models, essentially none of their original gas mass remains within a scale radius at $25 \mathrm{Myr}$. Thus, subsequent star formation does not occur.

2. For all M60 cooling models, only a few percent of the original gas mass remains within a scale radius at $25 \mathrm{Myr}$. No significant star formation can occur.

3. For the M65 models, all of the cooling scenarios retain $70 \%-80 \%$ of the original gas inside the virial radius at $25 \mathrm{Myr}$. This is sufficient for ongoing star formation. None of the adiabatic models retain more than $20 \%$ of the original gas.

4. In the borderline M65 cooling cases, off-centered models retain 2-3 times more gas within a scale radius, but the SN metals mix less efficiently with this gas.

5. For the M70 models, all of the gas survives an SN event and its radiative progenitor. We stress that we have not considered clustered multi-SN events, which can occur in these models.

6. Cooling is the most important factor governing gas retention within the virial radius at late times.

7. Clumpiness is far more important in the adiabatic case than in the cooling case for gas retention.

\section{DISCUSSION}

\subsection{Background}

The least massive galaxies lie at the bottom of the CDM hierarchy. Our focus here is not their role as building blocks in galaxy evolution, but what they can tell us about the yields of the first stars prior to the reionization epoch, and the following generations during and immediately after reionization (e.g., Bland-Hawthorn et al. 2010; Norris et al. 2010; Frebel \& Bromm 2012; Ritter et al. 2014). At least some of the first galaxies must have resided within low-mass halos. We anticipate that such systems, if they can be identified in 


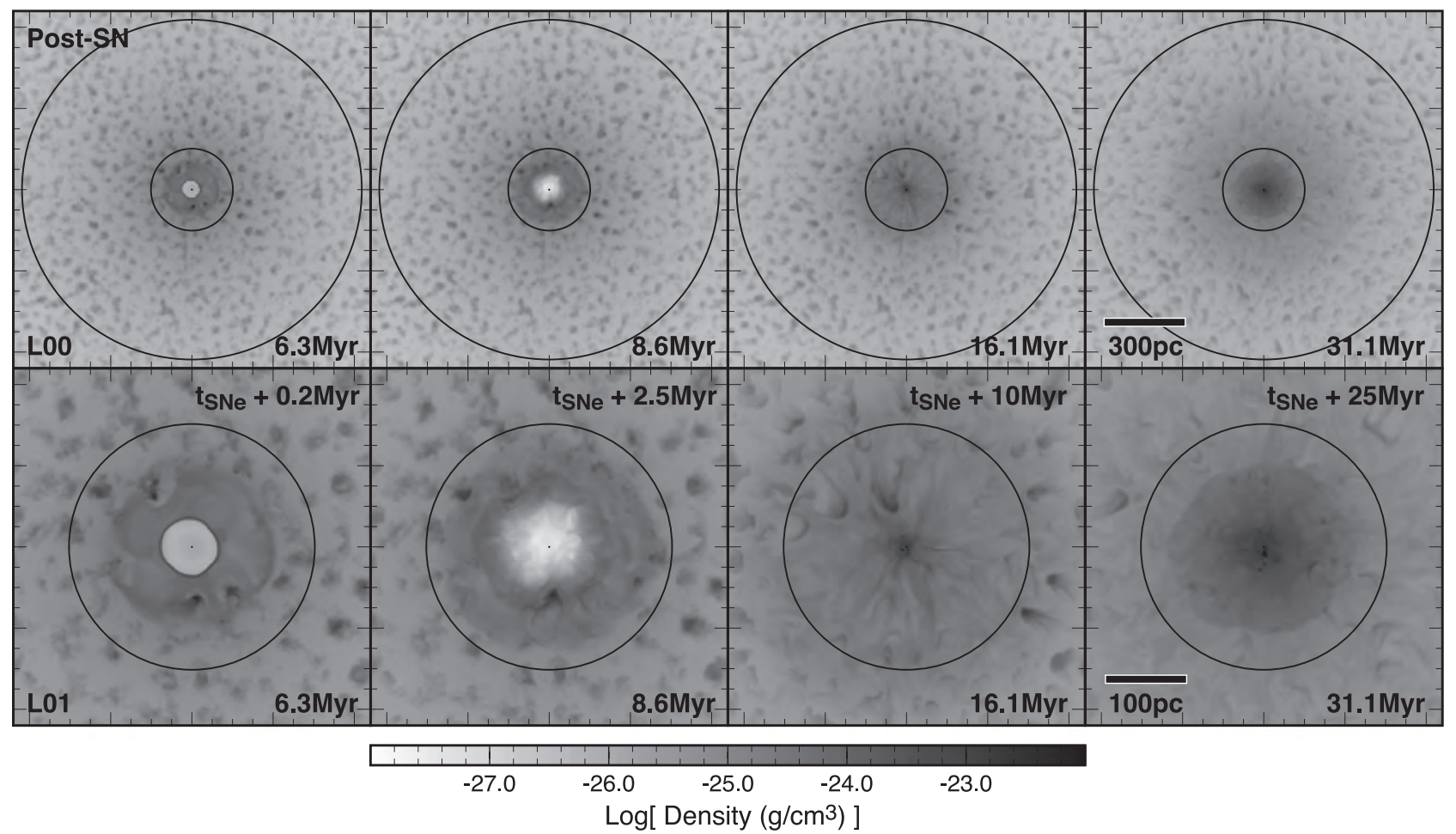

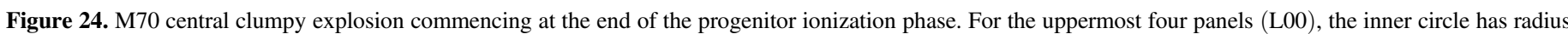

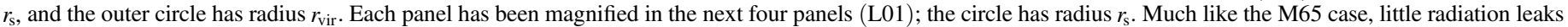

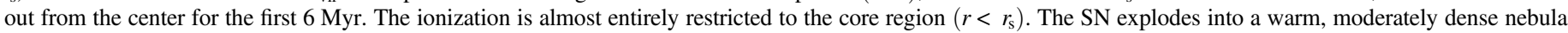

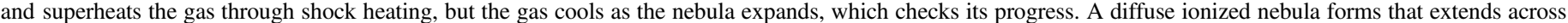

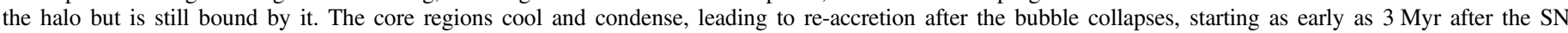

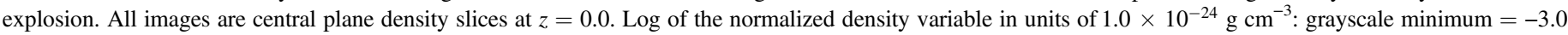
(white), maximum $=2.0$ (black). A scale bar in parsecs is shown.

the present-day universe, are likely to carry the most ancient chemical signatures because of their age and because of the relatively low number of enrichment events over cosmic time.

If we can identify such objects, it should be possible to obtain a relatively clean signature of the primordial yields from the earliest stellar generations. The star formation efficiency is greatly diminished in low-mass galaxies, thereby reducing the total number of enrichment events. There are likely to be instances where a large fraction of the metals are blown out of the galaxy, and this may obscure the signatures of earlier generations of stars. This can occur when the relatively rare $\mathrm{SNe}$ that go off in these galaxies are correlated in time. But we would still expect to find the signatures of a single enrichment event in at least some intrinsically lowmass galaxies.

We now discuss our models in light of two recent discoveries: UFDs (near field) identified in all-sky imaging surveys (Simon \& Geha 2007; Kirby et al. 2008), and very metal-poor DLAs (far field) identified along quasar sight lines (Erni et al. 2006; Cooke et al. 2010), although a more extensive discussion is given elsewhere (Webster et al. 2014, 2015a, 2015b). We refer the reader to Figures 1 and 2, which show that slow evolution is expected between the formation redshift at $z=10$, the epoch of the DLAs $(z=2-4)$, and present-day UFDs. The overall effect is additional dark matter that is mostly accreted to the outer envelope of the dwarf, rather than onto the central cusp (Figure 4).

\subsection{Ultrafaint Dwarfs}

In recent years, the Sloan Digital Sky Survey has doubled the number of known dwarf galaxies that orbit the Galaxy (for a review, see Tolstoy et al. 2009), particularly at the low-mass end. These newly discovered dark-matter-dominated galaxies are uniformly very old and cover a wide range in luminosity down to $\sim 10^{3} L_{\odot}$. Unlike globular clusters, they show a wide range of eccentricities, which may indicate that some are undergoing tidal disruption (Martin et al. 2008). Objects less luminous than $10^{5} L_{\odot}$ are referred to as UFDs.

There has been extensive discussion concerning the integrated masses of UFDs as observed today (Simon \& Geha 2007; Martin et al. 2008; Strigari et al. 2008; Bovill \& Ricotti 2009; Wolf et al. 2010; Frebel et al. 2014). Some studies quote a characteristic UFD mass within 300 pc, i.e., $\mathrm{M}_{300} \approx 10^{7} M_{\odot}$ (Strigari et al. 2008; Walker et al. 2009), although this is an arbitrary physical scale that is not easily related to well-known scaling relations. The characteristic virial mass $M_{\text {vir }}$ today is highly uncertain, with estimates ranging from $10^{8}$ to $10^{9} M_{\odot}$. For a dark halo described by the NFW profile, the mass within a fixed radius gives the mass within any other radius, assuming that this is an accurate description of the underlying gravitational potential. For our work, we need to know the virial mass when the UFD first formed. The halo parameters in cosmological simulations have a strong dependence with redshift, making any estimates rather uncertain (Power et al. 2003; Duffy et al. 2008). We have adopted $M_{\text {vir }} \lesssim$ $10^{7} M_{\odot}$ because the anticipated total population of UFDs may be too large to support larger progenitor masses. 


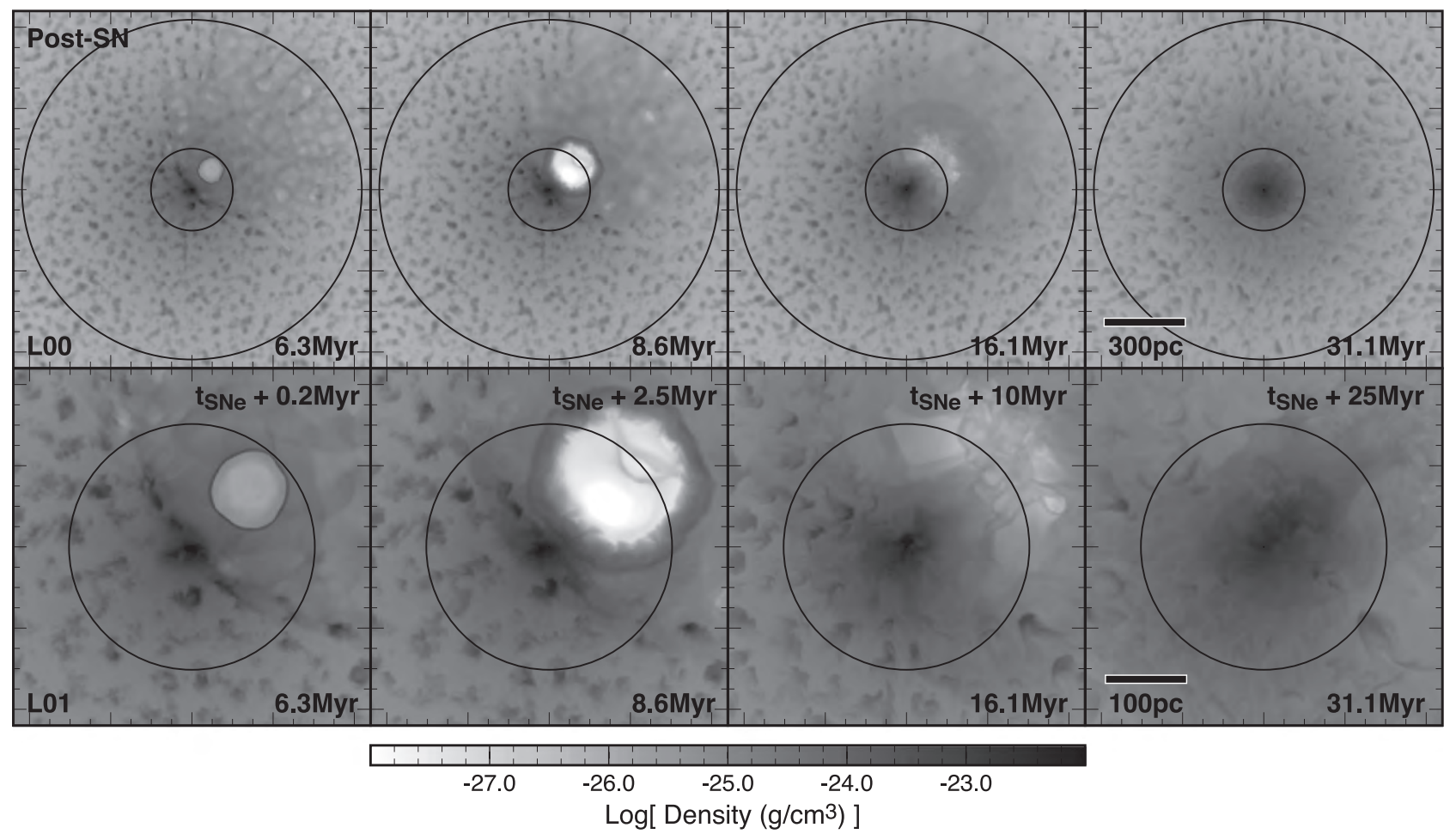

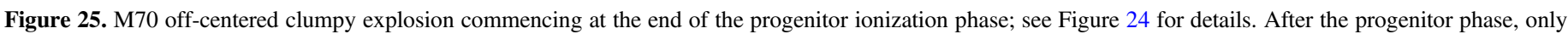

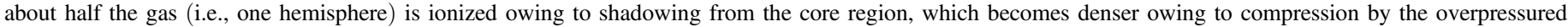

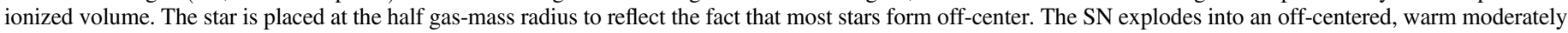

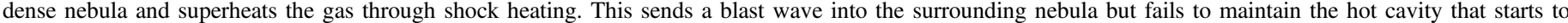

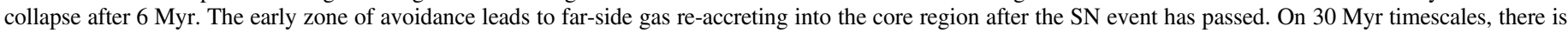
essentially no gas loss from the dark halo.

The simulated "observables" from our models are broadly consistent with the most metal-poor UFDs (Webster et al. 2014, 2015a). The data for these systems are summarized by Frebel \& Bromm (2012, Figure 1). The metallicity range for our models is $-1.0<[\mathrm{Fe} / \mathrm{H}]<-4.0$ with a mean $[\alpha / \mathrm{Fe}]$ enhanced by a factor of a few with respect to the solar value depending on the IMF at the time of formation (Woosley \& Weaver 1995). We have begun to consider the $r$ - and $s$-process signatures in the gas, work that is to be presented in later papers. The internal kinematics of the baryons are less than $4 \mathrm{~km} \mathrm{~s}^{-1}$ within the inner scale radius, consistent with observations (Walker et al. 2009).

Brown et al. (2014) find that the star formation history of UFDs is marked by one or two bursts of star formation before truncation by a global event. By construction, such a history is consistent with our models. UFDs have stellar populations with mean metallicities in the range $-2.5<[\mathrm{Fe} / \mathrm{H}]<-2.0$ (Kirby et al. 2013). Within the framework of our models, this requires (i) 1-2 SN events in a low-mass halo, specifically the M65 event-centered models (Section 5.4); and (ii) 3-10 SN events in a starburst within a dark matter halo an order of magnitude more massive (e.g., M70). These cases can give rise to measurably distinct chemical histories, star formation histories, and star cluster populations. For example, $[\alpha / \mathrm{Fe}]$ is enhanced in starbursts relative to quiescent star-forming regions. Direct evidence for this comes from X-ray observations of starburst winds (Martin et al. 2002).

At the time of publication, we note that Oñorbe et al. (2015) have presented a new model for UFDs where the baryons reside within dark matter halos that are orders of magnitude more massive than we consider $\left(M_{\mathrm{vir}} \approx 3 \times 10^{9} M_{\odot}\right)$. Given that all of the baryons are retained within our M70 models, for the same initial gas fraction, we cannot easily distinguish their model from ours in terms of the predicted chemical abundances.

In summary, if UFD masses are largely unchanged since their early formation, we would expect these halos to show significant scatter in $[\mathrm{Fe} / \mathrm{H}]$ at low metallicity, and for some of the oldest stars to constrain the yields of early stellar generations (e.g., Frebel \& Bromm 2012).

\subsection{Very Metal-poor DLAs}

An interesting development is the recent discovery of very metal-poor DLAs along QSO sight lines (Pettini et al. 2008; Penprase et al. 2010). If these are protogalactic structures that have recently formed from the intergalactic medium (IGM), there is the prospect of identifying the chemical imprint of early generations of stars (e.g., Pettini et al. 2002). Interestingly, two of these systems with $[\mathrm{Fe} / \mathrm{H}] \sim-3$ may bear the hallmarks of early stellar enrichment (Erni et al. 2006; Cooke et al. 2010, 2015).

First, Erni et al. (2006) identify a DLA toward the QSO Q0913+072 $(z=2.785)$ with an iron abundance characteristic of the IGM at that redshift. The $\mathrm{C}, \mathrm{N}, \mathrm{O}, \mathrm{Al}$, and $\mathrm{Si}$ abundances show an odd-even effect reminiscent of the most metal-poor stars in the Galactic halo. This pattern is created in models where the neutron flux is low (e.g., Heger \& Woosley 2002), presumably owing to the low overall metal abundance. A more striking signature is the strong $[\mathrm{N} / \mathrm{H}]$ depletion, which Pettini et al. (2002) have argued is further evidence for a system that 
has recently formed from the IGM. Erni et al. (2006) argue that the abundances appear to agree with $10-50 M_{\odot}$ zero-metallicity Pop III models.

Second, Cooke et al. (2015) identify half a dozen DLA objects that resemble the properties of UFDs with mean $[\mathrm{Fe} / \mathrm{H}]$ in the range $-2.5<[\mathrm{Fe} / \mathrm{H}]<-2.0$ and line-of-sight dispersion velocities of $1<\sigma_{\mathrm{LOS}}<5 \mathrm{~km} \mathrm{~s}^{-1}$ corrected for thermal and turbulent broadening. These physical parameters are in line with our M65 and M70 models (Webster et al. 2015b).

Finally, we examined whether the line-of-sight properties of our models would qualify them as DLAs. For all very metalpoor DLAs, Cooke et al. (2015) infer the total mass of neutral gas to be $10^{4} \lesssim M_{\text {gas }} \lesssim 10^{7} M_{\odot}$ within a linear scale of $\lesssim 30-1300 \mathrm{pc}$, although their UFD candidates are at the low end of these ranges. There are no sight lines in the M55 models, and few sight lines in the M60 models, that reach the DLA threshold. But the M65 and M70 models fare better, with a significant fraction of sight lines within a radius of $\sim r_{\mathrm{s}}$ being classified as a DLA-type spectrum. Furthermore, the inferred gas metallicity of $[\mathrm{Fe} / \mathrm{H}] \sim-3$ falls well within our predicted range. This gas is mostly self-shielded from any external radiation and therefore does not require an ionization correction.

\section{CONCLUSIONS}

To investigate the issue of gas retention in the lowest-mass dark matter halos, we performed a series of experiments in the form of high-resolution radiative hydrodynamic simulations dominated by the gravitational potential of the dark matter. We find that the broad properties of UFDs and very metal-poor DLAs are consistent with the host halos having intrinsically low mass. Our calculations are based on the critical period a few Myr before the SN explodes, and up to $25 \mathrm{Myr}$ after the event. These cases are explored over much longer timescales ( $600 \mathrm{Myr})$ elsewhere, which allows us to consider the effect of subsequent SN events and the homogenizing effects of reaccreting gas dislodged by earlier events (Webster et al. 2014, 2015a, 2015b).

In summary, dark matter halos with virial masses as little as $3 \times 10^{6} M_{\odot}$ are able to retain some gas, a limit that is an order of magnitude lower than found previously (Mac Low \& Ferrara 1999). The effects of cooling, the clumpiness of the medium, and the position of the explosion were studied. Consistent with earlier work, the baryons do not survive in smooth adiabatic or smooth cooling models in the event of an SN. Models with radiative cooling, clumpy media, and offcentered explosions are most favorable to gas retention. Our highest-resolution simulations reveal why cooling is so effective in retaining gas compared to any other factor. In the early stages, the super-hot metal-enriched SN ejecta exhibit strong cooling, leading to much of the explosive energy being lost. This remains true regardless of the assumed metallicity floor at the time of the explosion.

An added complication is to consider asymmetric explosions in core-collapse $\mathrm{SNe}$ (Grefenstette et al. 2014) rather than the isotropic explosions considered here. But we believe that these do not lead to radically different outcomes when compared to the off-centered, isotropic SN models. To first order, the overall mixing is dictated by geometry such that an energetic pulse experiencing any asymmetry leads to broadly similar metallicity spreads. If confirmed by later work, this principle will greatly reduce the parameter space of future simulations. It remains to be seen whether asymmetric versus symmetric explosions lead to the ejection of different abundance yields.

We recognize that at the low-mass limit explored in our models, the predictions are subject to stochastic effects due to the rarity of SN events. Any assumed IMF will be poorly sampled in this limit. In rare instances, simultaneous SN events are statistically possible, but these are more likely in highermass halos than considered here. Conversely, it is conceivable that low-mass star formation can proceed at the adopted metallicity threshold of $[\mathrm{Fe} / \mathrm{H}]=-4$ (Bromm \& Yoshida 2011) such that no prior $\mathrm{SN}$ activity is required beyond the enrichment from the first stars. This leads to a small fraction of stars extending down to the metallicity floor, a signature observed in only a few cases, e.g., Bootes I (Gilmore et al. 2013) and Segue I (Norris et al. 2010).

This work has identified new lines of inquiry. In particular, the central gas densities are chosen to limit the gas mass inside one scale radius to about $10 \%$ of the dark matter mass on the same scale (Section 2). Such a highly concentrated baryon fraction is to be expected because the star formation efficiency is likely to be very low before the onset of metal (and therefore dust) production (Krumholz \& Dekel 2012). In future papers, we will consider higher baryon fractions, including those that dominate over the dark matter core. This requires us to include self-gravity in the Fyris code through the use of fast Fourier transforms over the refined adaptive grid. In these models, we treat the pre-SN phase with higher-resolution grids. We have already begun to implement self-consistent cooling arising from variable enrichment over the gas distribution. Our future models will include the formation of dust and molecules. A complete understanding of UFDs will need to include the details of the subhalo orbit within the evolving Galactic potential and the coronal gas distribution responsible for the gas stripping (Nichols et al. 2012, 2015).

J.B.H. is supported by an Australian Laureate Fellowship from the Australian Research Council (ARC). J.B.H. is indebted to Merton College, Oxford, for a Visiting Research Fellowship and to the Leverhulme Trust for a Visiting Professorship to Oxford. D.W. is funded by an Australian Postgraduate Award. We are grateful to the Kavli Institute at UC Santa Barbara for hosting us in the final stages of this work. We are indebted to Julio Navarro, Anna Frebel, Josh Simon, Marla Geha, Evan Kirby, and Charlie Conroy for insightful comments. We have benefited from numerous incisive comments and insights from an anonymous referee.

\section{APPENDIX A ABUNDANCES}

The host halo gas metallicity was taken to be $[\mathrm{Fe} / \mathrm{H}]=-4.0$. The abundances, by number relative to hydrogen $=1.0$, are shown in Table 7. The solar abundances are based on Asplund et al. (2005), with a mild alpha-element enhancement pattern: carbon $(\times 1.5)$, oxygen $(\times 5.0)$, magnesium $(\times 2.5)$, silicon $(\times 2.5)$, and calcium $(\times 2.5)(\mathrm{K} 13)$. Helium evolution follows $\left(\right.$ Dopita et al. 2006) $n_{\mathrm{He}} / n_{\mathrm{H}}=0.0737+0.0024 \mathrm{Z} / Z_{\odot}$.

The SN ejecta composition was modeled on the well-studied M25 SN (see Kobayashi et al. 2006; Nomoto et al. 2006). Using the Kobayashi compilation yields $2.79 M_{\odot}$ of oxygen in the $\sim 4.4 M_{\odot}$ of total metals ejected, with $0.072 M_{\odot}$ of Fe. 
Table 7

Gas Phase Abundances, $[\mathrm{Fe} / \mathrm{H}]=-4.0$, Neutral: $\mu=1.21$, Ionized: $\mu=0.59$

\begin{tabular}{lcccr}
\hline \hline Element & $\begin{array}{c}\text { SN25 } Z=0 \\
\text { Mass Yields }\end{array}$ & $\begin{array}{c}\text { SN25 } Z=0 \\
\text { Abundances }\end{array}$ & $\begin{array}{c}\text { "Primordial" } \\
\text { Abundances }\end{array}$ & $\begin{array}{r}\text { Solar } \\
\text { Ratios }\end{array}$ \\
\hline $\mathrm{H}(\mathrm{X})$ & 10.60 & 0.00 & 0.00 & 0.00 \\
$\mathrm{He}(\mathrm{Y})$ & 8.03 & -0.72 & $-1.13^{\mathrm{a}}$ & -1.01 \\
Metals $(Z)$ & 4.41 & -1.62 & -6.27 & -3.09 \\
\hline $\mathrm{C}$ & $2.94 \mathrm{E}-01$ & -2.63 & -7.28 & -4.10 \\
$\mathrm{~N}$ & $5.91 \mathrm{E}-04$ & -5.40 & -10.05 & -4.79 \\
$\mathrm{O}$ & $2.79 \mathrm{E}+00$ & -1.78 & -6.43 & -3.34 \\
$\mathrm{Ne}$ & $5.33 \mathrm{E}-01$ & -2.60 & -7.25 & -3.91 \\
$\mathrm{Na}$ & $1.03 \mathrm{E}-03$ & -5.37 & -10.02 & -5.75 \\
$\mathrm{Mg}$ & $1.20 \mathrm{E}-01$ & -3.33 & -7.98 & -4.42 \\
$\mathrm{Al}$ & $8.08 \mathrm{E}-04$ & -5.55 & -10.19 & -5.61 \\
$\mathrm{Si}$ & $3.51 \mathrm{E}-01$ & -2.92 & -7.57 & -4.49 \\
$\mathrm{~S}$ & $1.86 \mathrm{E}-01$ & -3.26 & -7.91 & -4.79 \\
$\mathrm{Ar}$ & $3.14 \mathrm{E}-02$ & -4.13 & -8.78 & -5.20 \\
$\mathrm{Ca}$ & $2.48 \mathrm{E}-02$ & -4.23 & -8.88 & -5.64 \\
$\mathrm{Fe}$ & $7.38 \mathrm{E}-02$ & -3.90 & -8.55 & -4.55 \\
$\mathrm{Ni}$ & $4.42 \mathrm{E}-04$ & -6.15 & -10.79 & -5.68 \\
\hline
\end{tabular}

Note.

${ }^{\mathrm{a}}$ Helium from BB nucleosynthesis, metals scaled to $[\mathrm{Fe} / \mathrm{H}]=-4.0$.

Table 7 shows the SN mass yields, and the abundances by number, for the ejecta and ambient medium, with a final column showing solar abundances ratios for reference.

\section{APPENDIX B \\ FRACTAL INTERSTELLAR MEDIUM}

\section{B.1. Lognormal Density Distribution}

We use a lognormal distribution to describe the single-point statistics of the density field of our non-uniform ISM. The lognormal distribution is a skewed continuous probability distribution. Unlike the normal distribution, it has a non-zero skewness, variable kurtosis, and in general the mode, median, and mean are unequal.

The lognormal distribution appears to be a nearly universal property of isothermal turbulent media in experimental, numerical, and analytical studies (e.g., Pumir 1994; Nordlund et al. 1999; Warhaft 2000). Moreover, it is encouraging that the lognormal distribution is the limiting distribution for the product of random increments, in the same way that the normal distribution plays that role for additive random increments. It is thus compatible, at least conceptually, with a generic cascading process consisting of repeated folding and stretching.

With a lognormal distribution, which is on average isotropic, the natural logarithm of the ISM density field is a Gaussian that has a mean $m$ and variance $s^{2}$. The probability density function for the lognormal distribution of the mass density $\rho$ is

$$
P(\rho)=\frac{1}{s \sqrt{2 \pi} \rho} \exp \left[\frac{-(\ln \rho-m)^{2}}{2 s^{2}}\right] .
$$

The mean $\mu$ and variance $\sigma^{2}$ of the density are given by

$$
\begin{aligned}
\mu & =\exp \left[m+s^{2} / 2\right] . \\
\sigma_{F}^{2} & =\mu^{2}\left(\exp \left[s^{2}\right]-1\right) .
\end{aligned}
$$

In these simulations we adopt $\mu=1.0, \sigma_{F}^{2}=5.0$ as our standard lognormal distribution. With these parameters, densities below the mean compose one-quarter of the mass and occupy three-quarters of the volume, and the mean is approximately 20 times the mode. See Sutherland \& Bicknell (2007) for more details.

\section{B.2. Power-law Density Structure}

The two-point structure of a homogeneous turbulent medium may be described in Fourier space. We denote the Fourier transform of the density $\rho(\boldsymbol{r})$ by $F(\boldsymbol{k})$ (where $\boldsymbol{k}$ is the wavenumber vector). The isotropic power spectrum $D(k)$ is the integral over solid angle in Fourier space of the spectral density $F(\boldsymbol{k}) F^{*}(\boldsymbol{k})$. In three dimensions,

$$
D(k)=\int k^{2} F(\boldsymbol{k}) F^{*}(\boldsymbol{k}) d \Omega .
$$

Even if the spectral density is anisotropic, the angular integral averages the spectral density into a one-dimensional function of $\boldsymbol{k}$ only. For a power-law dependence on $\boldsymbol{k}$, $D(\boldsymbol{k}) \propto \boldsymbol{k}^{-\beta}$, and $\beta=5 / 3$, the spectrum is referred to as Kolmogorov turbulence.

In order to simultaneously achieve lognormal single-point statistics and a power-law self-similar structure, we have implemented the practical method developed by atmospheric scientists, for constructing two- and three-dimensional terrestrial cloud models, which are used in radiative transfer calculations (Lewis \& Austin 2002).

The remaining choice in this procedure is to select the range of wave numbers over which to generate the fractal, in particular the minimum wavenumber $\boldsymbol{k}_{\min }$, which determines the largest structure scale in the resulting fractal with respect to the spatial grid. The main domain of interest on the intermediate level in the simulations covers at least two dark matter scale radii in each model. We choose $\boldsymbol{k}_{\min }=8$ over this domain, corresponding to approximately two cloud structures per scale radius. On the upper level, the domain covers three times the extent, and the fractal is extended in a $\boldsymbol{k}_{\min }=24$ model. The lowest level is initially populated with a simple resampled version of the intermediate-level density field. Each level of the simulations is sampled in $216^{3}$ cells, and so even the coarse $\boldsymbol{k}_{\min }=24$ upper level has well-resolved small clouds.

\section{B.3. Equilibrium Turbulent Distribution}

Here we consider purely spherical distributions, neglecting rotational formation of disk structures. The reasons are twofold. First, flattening due to rotation will provide a natural axis for the escape of the SN energy and potentially greatly reduce the efficiency of the SN in evacuating the region of potentially starforming gas. We are looking for a conservative estimate to the lowest-mass halo that retains gas, and so we wish to retain spherical symmetry, in order to maximize the potential interaction between the host ISM and the SNe. Second, it is less clear that these very small halos include significant angular momentum, and so a non-rotating model is the simplest applicable one.

Taking the mean global temperature of the ISM to be $\tilde{T}$ and the line-of-sight turbulent velocity dispersion to be $\sigma_{t}$, the mean density of gas in the potential, $\Phi$, is given by (Sutherland \& 
Bicknell 2007)

$$
\frac{\rho(r)}{\rho(0)}=\exp \left[-\frac{\sigma_{D}^{2}}{\sigma_{g}^{2}} \Phi(r)\right]^{\beta} .
$$

where we take $e=0, \sigma_{g}^{2}=\sigma_{t}^{2}+k \tilde{T} / \mu \mathrm{m}$, and $\sigma_{D}$ is the velocity dispersion of the dark matter, assuming also that $\tilde{T}$ is the mean ISM temperature and $\sigma_{t}$ is constant throughout the ISM.

This mean global density distribution is multiplied by the fractal generated above, giving a clumpy distribution with the same mean global properties, and which is stable enough over the timescale of the simulations to remain static in the absence of an $\mathrm{SN}$ explosion.

\section{REFERENCES}

Abel, T., Bryan, G. L., \& Norman, M. L. 2000, ApJ, 540, 39

Abel, T., Bryan, G. L., \& Norman, M. L. 2002, Sci, 295, 93

Ahn, K., \& Shapiro, P. R. 2007, MNRAS, 375, 881

Allen, M. G., Groves, B. A., Dopita, M. A., Sutherland, R. S., \& Kewley, L. J. 2008, ApJS, 178, 20

Arnaud, M. 2009, A\&A, 500, 103

Asplund, M., Grevesse, N., \& Sauval, A. J. 2005, in ASP Conf. Ser. 336, Cosmic Abundances as Records of Stellar Evolution and Nucleosynthesis, ed. T. G. Barnes, III \& F. N. Bash (San Francisco, CA: ASP), 25

Barkana, R., \& Loeb, A. 1999, ApJ, 523, 54

Belokurov, V., Zucker, D. B., Evans, N. W., et al. 2006, ApJL, 647, L111

Belokurov, V., Zucker, D. B., Evans, N. W., et al. 2007, ApJ, 654, 897

Benítez-Llambay, A., Navarro, J. F., Abadi, M. G., et al. 2013, ApJL, 763, L4

Bigiel, F., Leroy, A., Walter, F., et al. 2008, AJ, 136, 2846

Bland-Hawthorn, J., Karlsson, T., Sharma, S., Krumholz, M., \& Silk, J. 2010, ApJ, 721, 582

Bland-Hawthorn, J., Sutherland, R., Agertz, O., \& Moore, B. 2007, ApJL, 670, L109

Bovill, M. S., \& Ricotti, M. 2009, ApJ, 693, 1859

Bromm, V., Ferrara, A., Coppi, P. S., \& Larson, R. B. 2001, MNRAS, 328, 969

Bromm, V., \& Yoshida, N. 2011, ARA\&A, 49, 373

Brown, T. M., Tumlinson, J., Geha, M., et al. 2012, ApJL, 753, L21

Brown, T. M., Tumlinson, J., Geha, M., et al. 2014, ApJ, 796, 91

Bullock, J. S., Kravtsov, A. V., \& Weinberg, D. H. 2001, ApJ, 548, 33

Bullock, J. S., Stewart, K. R., Kaplinghat, M., Tollerud, E. J., \& Wolf, J. 2010, ApJ, 717, 1043

Busha, M. T., Alvarez, M. A., Wechsler, R. H., Abel, T., \& Strigari, L. E. 2010, ApJ, 710, 408

Castelli, F., \& Kurucz, R. L. 2004, arXiv:astro-ph/0405087

Cavaliere, A., \& Fusco-Femiano, R. 1976, A\&A, 49, 137

Clark, P. C., Glover, S. C. O., \& Klessen, R. S. 2008, ApJ, 672, 757

Clark, P. C., Glover, S. C. O., Klessen, R. S., \& Bromm, V. 2011, ApJ, 727, 110

Cooke, R., Pettini, M., Steidel, C. C., et al. 2010, MNRAS, 409, 679

Cooke, R., Pettini, M., Steidel, C. C., Rudie, G. C., \& Jorgenson, R. A. 2011a, MNRAS, 412, 1047

Cooke, R., Pettini, M., Steidel, C. C., Rudie, G. C., \& Nissen, P. E. 2011b, MNRAS, 417, 1534

Cooke, R. J., Pettini, M., \& Jorgenson, R. A. 2015, ApJ, 800, 12

Cooper, J. L., Bicknell, G. V., Sutherland, R. S., \& Bland-Hawthorn, J. 2008, ApJ, 674, 157

Correa, C. A., Wyithe, J. S. B., Schaye, J., \& Duffy, A. R. 2015, arXiv: 1502.00391

Dalla Vecchia, C., \& Schaye, J. 2012, MNRAS, 426, 140

Dijkstra, M., Haiman, Z., Rees, M. J., \& Weinberg, D. H. 2004, ApJ, 601, 666

Dopita, M. A., Fischera, J., Sutherland, R. S., et al. 2006, ApJS, 167, 177

Duffy, A. R., Schaye, J., Kay, S. T., \& Dalla Vecchia, C. 2008, MNRAS, 390, L64

Efstathiou, G. 1992, MNRAS, 256, 43P

Einasto, J. 1965, TrAlm, 5, 87

Ekström, S., Meynet, G., \& Maeder, A. 2006, in ASP Conf. Ser. 353, Stellar Evolution at Low Metallicity: Mass Loss, Explosions, Cosmology, ed. H. J. G. L. M. Lamers et al. (San Francisco, CA: ASP), 141

Elmegreen, B. G., \& Scalo, J. 2004, ARA\&A, 42, 211
Erni, P., Richter, P., Ledoux, C., \& Petitjean, P. 2006, A\&A, 451, 19

Frebel, A., \& Bromm, V. 2012, ApJ, 759, 115

Frebel, A., Simon, J. D., \& Kirby, E. N. 2014, ApJ, 786, 74

Gao, L., Yoshida, N., Abel, T., et al. 2007, MNRAS, 378, 449

Georgy, C., Ekström, S., Meynet, G., et al. 2012, A\&A, 542, A29

Gilmore, G., Norris, J. E., Monaco, L., et al. 2013, ApJ, 763, 61

Grefenstette, B. W., Harrison, F. A., Boggs, S. E., et al. 2014, Natur, 506, 339

Greif, T. H., Springel, V., White, S. D. M., et al. 2011, ApJ, 737, 75

Hargis, J. R., Willman, B., \& Peter, A. H. G. 2014, ApJL, 795, L13

Hayashi, E., Navarro, J. F., Power, C., et al. 2004, MNRAS, 355, 794

Heger, A., \& Woosley, S. E. 2002, ApJ, 567, 532

Heiles, C. 2004, in ASP Conf. Ser. 323, Star Formation in the Interstellar Medium: In Honor of David Hollenbach, ed. D. Johnstone et al. (San Francisco, CA: ASP), 79

Hurley-Keller, D., Mateo, M., \& Nemec, J. 1998, AJ, 115, 1840

Ikeuchi, S. 1986, Ap\&SS, 118, 509

Irwin, M. J., Belokurov, V., Evans, N. W., et al. 2007, ApJL, 656, L13

Jeon, M., Pawlik, A. H., Bromm, V., \& Milosavljević, M. 2014, MNRAS, 444, 3288

Kang, H., \& Shapiro, P. R. 1992, ApJ, 386, 432

Karlsson, T., Bromm, V., \& Bland-Hawthorn, J. 2013, RvMP, 85, 809

Kennedy, R., Frenk, C., Cole, S., \& Benson, A. 2014, MNRAS, 442, 2487

Kennicutt, R. C., Jr. 1989, ApJ, 344, 685

Kirby, E. N., Cohen, J. G., Guhathakurta, P., et al. 2013, ApJ, 779, 102

Kirby, E. N., Simon, J. D., Geha, M., Guhathakurta, P., \& Frebel, A. 2008, ApJL, 685, L43

Kobayashi, C., Umeda, H., Nomoto, K., Tominaga, N., \& Ohkubo, T. 2006, ApJ, 653, 1145

Krumholz, M. R., \& Dekel, A. 2012, ApJ, 753, 16

Kudritzki, R. P. 2002, ApJ, 577, 389

Kudritzki, R.-P. 2005, in ASP Conf. Ser. 332, The Fate of the Most Massive Stars, ed. R. Humphreys \& K. Stanek (San Francisco, CA: ASP), 239

Lewis, G. M., \& Austin, P. H. 2002, in Proc. XI Conf. on Atmospheric Radiation (Boston, MA: American Meteorological Assocation), http://ams. confex.com/ams/11AR11CP/11cldphy/abstracts/42772.htm

Mac Low, M., \& Ferrara, A. 1999, ApJ, 513, 142

Martin, C. L., Kobulnicky, H. A., \& Heckman, T. M. 2002, ApJ, 574, 663

Martin, N. F., de Jong, J. T. A., \& Rix, H. 2008, ApJ, 684, 1075

McGaugh, S. S., Schombert, J. M., de Blok, W. J. G., \& Zagursky, M. J. 2010, ApJL, 708, L14

Meynet, G., \& Maeder, A. 2000, A\&A, 361, 101

Meynet, G., \& Maeder, A. 2002, A\&A, 390, 561

Nichols, M., \& Bland-Hawthorn, J. 2009, ApJ, 707, 1642

Nichols, M., Lin, D., \& Bland-Hawthorn, J. 2012, ApJ, 748, 149

Nichols, M., Revaz, Y., \& Jablonka, P. 2015, arXiv:1503.05190

Nomoto, K., Tominaga, N., Umeda, H., Kobayashi, C., \& Maeda, K. 2006 , NuPhA, 777, 424

Nordlund, A. K., \& Padoan, P. 1999, in Interstellar Turbulence, ed. J. Franco \& A. Carraminana (Cambridge: Cambridge Univ. Press), 218

Norman, M. L. 2010, in AIP Conf. Ser. 1294, The First Stars and Galaxies: Challenges for the Next Decade, ed. D. J. Whalen, V. Bromm, \& N. Yoshida (Melville, NY: AIP), 17

Norris, J. E., Wyse, R. F. G., Gilmore, G., et al. 2010, ApJ, 723, 1632

Ocvirk, P., \& Aubert, D. 2011, MNRAS, 417, L93

Ocvirk, P., Aubert, D., Chardin, J., et al. 2013, ApJ, 777, 51

Okamoto, T., Gao, L., \& Theuns, T. 2008, MNRAS, 390, 920

Okrochkov, M., \& Tumlinson, J. 2010, ApJL, 716, L41

Oñorbe, J., Boylan-Kolchin, M., Bullock, J. S., et al. 2015, arXiv:1502.02036

O'Shea, B. W., \& Norman, M. L. 2007, ApJ, 654, 66

Parkinson, H., Cole, S., \& Helly, J. 2008, MNRAS, 383, 557

Peebles, P. J. E., \& Dicke, R. H. 1968, ApJ, 154, 891

Penprase, B. E., Prochaska, J. X., Sargent, W. L. W., Toro-Martinez, I., \& Beeler, D. J. 2010, ApJ, 721, 1

Pettini, M., Ellison, S. L., Bergeron, J., \& Petitjean, P. 2002, A\&A, 391, 21

Pettini, M., Zych, B. J., Steidel, C. C., \& Chaffee, F. H. 2008, MNRAS, 385, 2011

Planck Collaboration, Ade, P. A. R., Aghanim, N., et al. 2014, A\&A, 571, A16 Podsiadlowski, P., Nomoto, K., Maeda, K., et al. 2002, ApJ, 567, 491

Power, C., Navarro, J. F., Jenkins, A., et al. 2003, MNRAS, 338, 14

Pumir, A. 1994, PhFl, 6, 3974

Rees, M. J. 1986, MNRAS, 218, 25P

Ricotti, M., \& Gnedin, N. Y. 2005, ApJ, 629, 259

Ritter, J. S., Safranek-Shrader, C., Gnat, O., Milosavljević, M., \& Bromm, V. 2012, ApJ, 761, 56

Ritter, J. S., Sluder, A., Safranek-Shrader, C., Milosavljevic, M., \& Bromm, V. 2014, arXiv:1408.0319 
Salvadori, S., \& Ferrara, A. 2009, MNRAS, 395, L6

Salvadori, S., \& Ferrara, A. 2012, MNRAS, 421, L29

Scalo, J., \& Elmegreen, B. G. 2004, ARA\&A, 42, 275

Schaerer, D. 2003, A\&A, 397, 527

Schneider, R., Ferrara, A., Natarajan, P., \& Omukai, K. 2002, ApJ, 571, 30

Schneider, R., \& Omukai, K. 2010, MNRAS, 402, 429

Sharp, R. G., \& Bland-Hawthorn, J. 2010, ApJ, 711, 818

Simon, J. D., \& Geha, M. 2007, ApJ, 670, 313

Springel, V., Wang, J., Vogelsberger, M., et al. 2008, MNRAS, 391, 1685

Sternberg, A., McKee, C. F., \& Wolfire, M. G. 2002, ApJS, 143, 419

Strigari, L. E., Koushiappas, S. M., Bullock, J. S., et al. 2008, ApJ, 678, 614

Susa, H., \& Umemura, M. 2000, ApJ, 537, 578

Susa, H., \& Umemura, M. 2004a, ApJ, 600, 1

Susa, H., \& Umemura, M. 2004b, ApJL, 610, L5

Sutherland, R. S. 2010, Ap\&SS, 327, 173

Sutherland, R. S., \& Bicknell, G. V. 2007, ApJS, 173, 37

Sutherland, R. S., Bicknell, G. V., \& Dopita, M. A. 1993, ApJ, 414, 510

Tajiri, Y., \& Umemura, M. 1998, ApJ, 502, 59

Thornton, K., Gaudlitz, M., Janka, H.-T., \& Steinmetz, M. 1998, ApJ, 500, 95
Tolstoy, E., Hill, V., \& Tosi, M. 2009, ARA\&A, 47, 371

Tsuribe, T., \& Omukai, K. 2008, ApJL, 676, L45

Umeda, H., \& Nomoto, K. 2003, Natur, 422, 871

Walker, M. G., Mateo, M., Olszewski, E. W., et al. 2009, ApJ, 704, 1274

Walsh, S. M., Jerjen, H., \& Willman, B. 2007, ApJL, 662, L83

Warhaft, Z. 2000, AnRFM, 32, 203

Weaver, R., McCray, R., Castor, J., Shapiro, P., \& Moore, R. 1977, ApJ, 218, 377

Webster, D., Bland-Hawthorn, J., \& Sutherland, R. 2015a, ApJL, 799, L21

Webster, D., Bland-Hawthorn, J., \& Sutherland, R. S. 2015b, ApJ, 804, 110

Webster, D., Sutherland, R., \& Bland-Hawthorn, J. 2014, ApJ, 796, 11

Weisz, D. R., Dolphin, A. E., Skillman, E. D., et al. 2014, ApJ, 789, 148

Willman, B., Dalcanton, J. J., Martinez-Delgado, D., et al. 2005, ApJL, 626, L85

Wise, J. H., \& Abel, T. 2008, ApJ, 685, 40

Wise, J. H., Turk, M. J., \& Abel, T. 2008, ApJ, 682, 745

Wolf, J., Martinez, G. D., Bullock, J. S., et al. 2010, MNRAS, 406, 1220

Woosley, S. E., \& Weaver, T. A. 1995, ApJS, 101, 181

Yoshida, N., Omukai, K., Hernquist, L., \& Abel, T. 2006, ApJ, 652, 6

Zucker, D. B., Belokurov, V., Evans, N. W., et al. 2006, ApJL, 643, L103 